Publ. Mat. 63 (2019), 3-79

DOI: 10.5565 /PUBLMAT6311901

\title{
ASYMPTOTIC EXPANSIONS AND SUMMABILITY WITH RESPECT TO AN ANALYTIC GERM
}

\author{
Jorge Mozo Fernández and Reinhard SchäFke
}

\begin{abstract}
In a previous article [CMS], monomial asymptotic expansions, Gevrey asymptotic expansions, and monomial summability were introduced and applied to certain systems of singularly perturbed differential equations. In the present work, we extend this concept, introducing (Gevrey) asymptotic expansions and summability with respect to a germ of an analytic function in several variables - this includes polynomials. The reduction theory of singularities of curves and monomialization of germs of analytic functions are crucial to establish properties of the new notions, for example a generalization of the Ramis-Sibuya theorem for the existence of Gevrey asymptotic expansions. Two examples of singular differential equations are presented for which the formal solutions are shown to be summable with respect to a polynomial: one ordinary and one partial differential equation.
\end{abstract}

2010 Mathematics Subject Classification: Primary: 41A60.

Key words: Asymptotic expansions, summability.

\section{Introduction}

The concept of asymptotic expansion for complex functions in one variable is well established and widely used since Poincaré, in order to give a meaning to divergent formal power series that appear as solutions of different functional equations, and to understand the behavior near singular points of analytic solutions and other special functions. We mention only the books of W. Wasow [Was1] and F. W. J. Olver [Olv].

The closely related notion of summability was introduced to provide in a unique way analytic functions having certain asymptotic expansions. In one complex variable, it has been extensively used in such different fields as ordinary differential equations, the analytic classification of formal objects, and some classes of singularly perturbed differential equations and partial differential equations.

The first author was partially supported by the Spanish national project MTM201015471. The second author was supported in part by grants of the French National Research Agency (ref. ANR-10-BLAN 0102 and ANR-11-BS01-0009). 
For asymptotic expansions in several variables, different approaches exist in the literature. Let us mention the approaches of R. Gérard and Y. Sibuya $[\mathbf{G S}]$, who treated some class of Pfaffian systems, and a more powerful one by H. Majima $[\mathbf{M a j}]$. This last author introduced the concept of strong asymptotic expansion in polysectors in order to study several classes of singularly perturbed differential equations and integrable connections.

Several problems suggested asymptotic expansions in several variables in which monomials $x^{p} y^{q}$ are crucial: W. Wasow [Was2] studied equations of the form

$$
\varepsilon^{h} x^{-k} \frac{d \mathbf{y}}{d x}=A(x, \varepsilon) \mathbf{y}
$$

where $h, k \in \mathbb{N}$ and $A(x, \varepsilon)$ is a matrix holomorphic near $\varepsilon=0$ and $x=\infty$. They are singular both in the variable $x$ and in the parameter $\varepsilon$. J. Martinet and J.-P. Ramis $[\mathbf{M R}]$ studied the analytic classification of resonant singularities of holomorphic foliations in two variables. The formal normal form involved the monomial $u=x^{p} y^{q}$. The normalizing transformations are $(k, p, q)$-summable in the following sense: they are locally defined as

$$
(x, y) \mapsto(x \exp \{q h(x, y)\}, y \exp \{-p h(x, y)\}),
$$

where $h(x, y)=f\left(x^{p} y^{q}\right)(x, y)$ and $u \mapsto f(u) \in \mathbb{C}\{x, y\}$ is obtained as the $k$-sum of an element $\tilde{f} \in \mathbb{C}\{x, y\} \llbracket u \rrbracket, k$-summable in the variable $u$ with coefficients in $\mathbb{C}\{x, y\}$. L. Stolovitch [Sto] used a similar construction in $n \geq 2$ variables.

These examples lead M. Canalis-Durand and the authors [CMS] to a detailed investigation of the concept of monomial asymptotic expansion in two variables. One possible definition is the above one given by $[\mathbf{M R}] ;[\mathbf{C M S}]$ gives a more algorithmical definition. In the case of $p=q=1$, a power series $f(x, y)$ is $(k, 1,1)$-summable if, rewritten $f(x, y)=\sum_{n=0}^{\infty}\left(a_{n}(x)+b_{n}(y)\right)(x y)^{n}$, all series $a_{n}(x), b_{n}(y)$ have a common radius of convergence $R>0$, and for sufficiently small $r>0$, the series $T f(u)=\sum_{n} c_{n} u^{n} \in \mathcal{O}_{b}\left(D(0, r)^{2}\right) \llbracket u \rrbracket$ is $k$-summable, where $c_{n}(x, y)=a_{n}(x)+b_{n}(y),|x|<r,|y|<r$ are elements of the Banach space $\mathcal{O}_{b}\left(D(0, r)^{2}\right)$ of bounded holomorphic functions on $D(0, r)^{2}$. [CMS] applied this definition to doubly singular ordinary differential equations of the form

$$
\varepsilon^{\sigma} x^{r+1} \frac{d \mathbf{y}}{d x}=f(x, \varepsilon, \mathbf{y}),
$$

where $f(0,0, \mathbf{0})=\mathbf{0}$ and $\frac{\partial f}{\partial \mathbf{y}}(0,0, \mathbf{0})$ is invertible.

Returning to the problem of classification of holomorphic foliations, observe that resonant ones are some of the models that appear as a final step in the reduction of singularities of holomorphic foliations in 
dimension two, in a situation in which the set of separatrices and the divisor have normal crossings. Some examples of vector fields with nilpotent linear part leading to foliations without normal crossings have been previously studied. For instance, F. Loray [Lor] considered generic perturbations of the system $\dot{x}=2 y, \dot{y}=3 x^{2}$ with hamiltonian $h=y^{2}-x^{3}$. He obtained a formal normal form involving formal power series in $h$. In $[\mathbf{C S}]$, it was shown that the normal form always contains summable series in $h$. In this context an extension of monomial summability to summability with respect to a polynomial will be useful. It is conjectured that there is a normalizing transformation that is summable with respect to $y^{2}-x^{3}$.

We choose to study asymptotic expansions with respect to a germ of an analytic function as it is no more difficult than asymptotic expansions with respect to a polynomial. Such a concept needs to behave properly with respect to blow-ups in both directions: properties of the asymptotic expansions must be preserved when you blow-up, and properties of the blow-up must give properties of the original asymptotic expansion. Let us remark here that in $[\mathbf{C a r}, \mathbf{C M}]$, the authors study the behaviour of monomial asymptotics under blow-ups in order to establish certain Tauberian theorems useful to study properties of Pfaffian systems.

The purpose of the present work is to introduce such concepts of asymptotic expansions and summability with respect to a germ of an analytic function in an arbitrary number of variables. We use blow-ups of centers of codimension two and ramifications, in the style of Rolin, Speissegger, and Wilkie [RSW], who, on their turn, follow the ideas of Bierstone and Milman $[\mathbf{B M}]$. The reader should note that we haven't used the full power of desingularization techniques - this undoubtedly deserves a further study. Throughout this work, different techniques are used, among them, induction on the number of steps needed to monomialize the analytic germ, a Generalized Weierstrass Division theorem, and Ramis-Sibuya theorems.

The structure of this work is as follows: In Section 2, we present some tools needed in the present work. Among them are a normalization result adapted from $[\mathbf{R S W}]$ and a Generalized Weierstrass Division theorem from $[\mathbf{A H V}]$. Both are proved here in a simplified version adapted to our needs. They are used to deal with bounded quotients of germs of analytic functions (i.e., for the elimination of indeterminacies, Lemma 2.2), and to rewrite a formal power series in terms of powers of a germ of an analytic function (Corollary 2.7). In Section 3, we recall some properties of classical and monomial asymptotic expansions and present the latter in a more general setting than in [CMS]. In particular, monomial 
asymptotic expansions are given for an arbitrary number of variables. Certain operators are defined, that transform, both in the formal and in the analytic setting, monomial asymptotic expansions into asymptotic expansion in one variable with coefficients in a Banach space - the inverse transformations are given by simple substitution operators.

Asymptotic expansions with respect to an arbitrary germ of an analytic function, which are the main object of this work, are defined in Section 4, and their main properties are established. In order to study them, we construct new operators analogous to the previous section that transform asymptotic expansions with respect to a germ into asymptotic expansions with respect to one variable with coefficients in some Banach space (see Theorem 4.9). In the analytic setting, these operators are constructed in Theorem 4.7; this is one of the main results of this section. Its rather technical proof is given in Section 5. It uses induction with respect to the number of steps needed to monomialize the analytic germ.

In Section 6, the behaviour with respect to blow-ups with centers of codimension two is established for the new concept of asymptotic expansion with respect to a germ of an analytic function. While a function $f$ having an asymptotic expansion with respect to a germ $P$ clearly also has a corresponding asymptotic expansion after blow-up, the converse is more interesting and it is proved in Theorem 6.8. For $P$-asymptotic series, i.e. series appearing as asymptotic expansions with respect to a germ $P$ for certain functions, we prove an analogous result (Theorem 6.10).

In Section 7, Gevrey asymptotic expansions with respect to an analytic germ are defined and investigated, and subsequently, summability with respect to such a germ. We also study the behavior of these concepts with respect to blow-ups.

Finally, in Section 8, we present two examples of singular differential equations for which the formal solutions are summable with respect to a polynomial: one ordinary and one partial differential equation. The examples suggest that asymptotic expansions with respect to an analytic germ will play an important role in these theories and in the theory of foliations. They also illustrate the application of our results, in particular concerning blow-ups, in proving summability.

Acknowledgments. The first author wishes to thank the University of Strasbourg and the second author wishes to thank the University of Valladolid for the hospitality during their visits while preparing this article. 


\section{Preliminaries}

2.1. Notation. The following notation will be used throughout this work. We fix an integer $d \geq 2$.

$D\left(\mathbf{0} ;\left(r_{1}, r_{2}, \ldots, r_{d}\right)\right):=\left\{\boldsymbol{x}=\left(x_{1}, x_{2}, \ldots, x_{d}\right) \in \mathbb{C}^{d} ;\left|x_{j}\right|<r_{j}\right.$, for $\left.j=1, \ldots, d\right\}$

is a polydisk around the origin. As an abbreviation, $D(\mathbf{0} ; \rho):=D(\mathbf{0} ;(\rho, \ldots$, $\rho))$. If $U$ is an open set, in $\mathbb{C}$ or in $\mathbb{C}^{d}, \mathcal{O}(U)=\mathcal{O}(U ; \mathbb{C})$ is the set of complex valued functions holomorphic on $U$, and $\mathcal{O}_{b}(U)$ the subset of bounded holomorphic functions. Analogously, if $E$ is a Banach space, $\mathcal{O}(U ; E)$ will denote the set of $E$-valued holomorphic functions on $U$, and $\mathcal{O}_{b}(U ; E)$ the set of bounded $E$-valued holomorphic functions. $\mathcal{O}=$ $\mathbb{C}\{\boldsymbol{x}\}=\mathbb{C}\left\{x_{1}, x_{2}, \ldots, x_{d}\right\}$ is the ring of germs of analytic functions at the origin (convergent power series), and $\hat{\mathcal{O}}=\mathbb{C} \llbracket \boldsymbol{x} \rrbracket$ the ring of formal power series. We denote by $\mathfrak{m}$ the maximal ideal of both (local) rings. There are natural inclusions

$$
\mathcal{O}_{b}(D(\mathbf{0} ; \rho)) \subseteq \mathcal{O}(D(\mathbf{0} ; \rho)) \subseteq \mathbb{C}\{\boldsymbol{x}\} \subseteq \mathbb{C} \llbracket \boldsymbol{x} \rrbracket,
$$

as well as the relation $\mathcal{O}=\cup_{\rho>0} \mathcal{O}_{b}(D(\mathbf{0} ; \rho))$ that we will not detail. For an element $f$ of one of those rings, $J(f)$ will be its power series at the origin, and $J_{m}(f)$ its $m$-jet, i.e., the polynomial of degree at most $m$ obtained from $J(f)$ deleting the terms of degree greater than $m$.

We use $\mathbb{P}_{\mathbb{C}}^{1}=\mathbb{C} \cup\{\infty\}$ with the usual topology. For $\boldsymbol{x}=\left(x_{1}, x_{2}, \ldots, x_{d}\right) \in$ $\mathbb{C}^{d}$ put $\boldsymbol{x}^{\prime}=\left(x_{2}, \ldots, x_{d}\right)$ and $\boldsymbol{x}^{\prime \prime}=\left(x_{3}, \ldots, x_{d}\right)$.

2.2. A normalization result. Our approach uses blow-ups at several essential points and we would like to recall the well known result we use. Our presentation follows that of $[\mathbf{R S W}]$ (who base their work on $[\mathbf{B M}]$ ); the results themselves are classical.

Following [RSW], we will only use blow-ups of codimension two smooth varieties and so we only recall this case. Assume that the center of the blow-up is $x_{1}=x_{2}=0$ and define

$$
M=\left\{\left(\left[u_{1}, u_{2}\right], \boldsymbol{t}\right) \in \mathbb{P}_{\mathbb{C}}^{1} \times \mathbb{C}^{d} ; u_{1} t_{2}=u_{2} t_{1}\right\}
$$

the blow-up variety and

$$
\begin{aligned}
b: M & \longrightarrow \mathbb{C}^{d} \\
\left(\left[u_{1}, u_{2}\right], t\right) & \longmapsto \boldsymbol{t}
\end{aligned}
$$

the blow-up map (shortly blow-up). $M$ is covered by affine charts, each one analytically equivalent to $\mathbb{C}^{d}$. In fact, identifying $\mathbb{P}_{\mathbb{C}}^{1} \cong \overline{\mathbb{C}}=\mathbb{C} \cup\{\infty\}$ as $[1, \xi] \equiv \xi \in \mathbb{C},[0,1] \equiv \infty$, we use the charts centered in $\xi \in \mathbb{C}$,

$$
\begin{aligned}
\phi_{\xi}: M_{\xi} & \longrightarrow \mathbb{C}^{d} \\
\left(\left[u_{1}, u_{2}\right], \boldsymbol{t}\right) & \longmapsto\left(\frac{u_{2}}{u_{1}}-\xi, t_{1}, \boldsymbol{t}^{\prime \prime}\right),
\end{aligned}
$$


where $M_{\xi}=M_{0}=\left\{\left(\left[u_{1}, u_{2}\right], \boldsymbol{t}\right) \in M ; u_{1} \neq 0\right\}$ and the chart at $\infty$

$$
\begin{aligned}
\phi_{\infty}: M_{\infty} & \longrightarrow \mathbb{C}^{d} \\
\left(\left[u_{1}, u_{2}\right], \boldsymbol{t}\right) & \longmapsto\left(\frac{u_{1}}{u_{2}}, t_{2}, \boldsymbol{t}^{\prime \prime}\right),
\end{aligned}
$$

where $M_{\infty}=\left\{\left(\left[u_{1}, u_{2}\right], \boldsymbol{t}\right) \in M ; u_{2} \neq 0\right\}$. Then the map $b$ is described by

$$
\begin{aligned}
b_{\xi}=b \circ \phi_{\xi}^{-1}: \mathbb{C}^{d} & \longrightarrow \mathbb{C}^{d} \\
\mathbf{v} & \longmapsto\left(v_{2},\left(\xi+v_{1}\right) v_{2}, \mathbf{v}^{\prime \prime}\right)
\end{aligned}
$$

in the chart at $\xi$ and by

$$
\begin{aligned}
b_{\infty}=b \circ \phi_{\infty}^{-1}: \mathbb{C}^{d} & \longrightarrow \mathbb{C}^{d} \\
\mathbf{v} & \longmapsto\left(v_{1} v_{2}, v_{2}, \mathbf{v}^{\prime \prime}\right),
\end{aligned}
$$

in the chart at $\infty$. The reason for our somewhat unusual choice of $\phi_{\xi}$ and hence $b_{\xi}$ will become clear in Section 6 ; otherwise the choice of charts is not important. For $k \in \mathbb{N}, k \geq 2$, we introduce the ramification

$$
\begin{aligned}
r_{k}: \mathbb{C}^{d} & \longrightarrow \mathbb{C}^{d} \\
\boldsymbol{t} & \longmapsto\left(t_{1}^{k}, \boldsymbol{t}^{\prime}\right) .
\end{aligned}
$$

We say that $f \in \mathcal{O}$ has normal crossings (at the origin) if there is a diffeomorphism $D \in \operatorname{Diff}\left(\mathbb{C}^{d}, \mathbf{0}\right)$ (i.e. its Jacobian at the origin is an invertible matrix) such that

$$
(f \circ D)(\boldsymbol{x})=x_{1}^{\ell_{1}} \cdots x_{d}^{\ell_{d}} U(\boldsymbol{x})
$$

with non-negative integers $\ell_{j}$ and a unit $U \in \mathcal{O}$, i.e. a germ satisfying $U(\mathbf{0}) \neq 0$.

It has been shown in $[\mathbf{R S W}]$ :

Lemma 2.1. There exists a function $h: \mathcal{O} \backslash\{0\} \rightarrow \mathbb{N}$ with the following properties:

(1) If $h(f)=0$ then $f$ has normal crossings.

(2) If $h(f)>0$ then there exists a diffeomorphism $D \in \operatorname{Diff}\left(\mathbb{C}^{d}, \mathbf{0}\right)$ such that either for all $\xi \in \mathbb{P}_{\mathbb{C}}^{1}$

$$
h\left(f \circ D \circ b_{\xi}\right)<h(f)
$$

or there exists $k \in \mathbb{N}, k \geq 2$ such that $h\left(f \circ D \circ r_{k}\right)<h(f)$.

Observe that this lemma can be applied simultaneously to a finite number of germs $f_{1}, f_{2}, \ldots, f_{r}$. It suffices to consider their product $f=$ $f_{1} f_{2} \cdots f_{r}$. Moreover, it is elementary (see $[\mathbf{R S W}]$ ) that $f_{1} f_{2} \cdots f_{r}$ has normal crossings if and only if every $f_{i}$ has normal crossings (with respect to a common diffeomorphism $D$ ).

In a more general setting (quasi-analytic classes), this result is proved in $[\mathbf{R S W}]$, following some general ideas adapted from $[\mathbf{B M}]$. In the analytic situation, the result is much easier. We sketch a proof for completeness, omitting most technical details. 
Proof: Use induction on $d$. Every $f \in \mathbb{C}\{x\}$ has normal crossings, so $h(f)=0$ and the result is trivial if $d=1$. Assume now that $d>1$, and let $n$ denote the order of $f$. By a linear change of variables, $f$ can be made $x_{d}$-regular of order $n$, that is $f\left(0, \ldots, 0, x_{d}\right)$ has valuation $n$. Indeed, as $f_{n}\left(\alpha_{1}, \ldots, \alpha_{d}\right) \neq 0$ (where $f_{n}$ is the homogeneous component of degree $n$ of $f$ ) for some sufficiently small vector $\boldsymbol{\alpha}$ with $\alpha_{d} \neq 0$, the function $\tilde{f}\left(t_{1}, \ldots, t_{d}\right)=f\left(t_{1}+\alpha_{1} t_{d}, \ldots, t_{d-1}+\alpha_{d-1} t_{d}, \alpha_{d} t_{d}\right)$ is regular.

If $f$ is $x_{d}$-regular, the Weierstrass Preparation theorem allows us to write $f(\boldsymbol{x})=\left(x_{d}^{n}+c_{1}\left(\boldsymbol{x}^{\prime}\right) x_{d}^{n-1}+\cdots+c_{n}\left(\boldsymbol{x}^{\prime}\right)\right) U(\boldsymbol{x})$ where $U \in \mathbb{C}\{\boldsymbol{x}\}$, $U(\mathbf{0}) \neq 0$, and $c_{1}, \ldots, c_{n}$ vanish at the origin. Hence it is sufficient to continue with the polynomial factor. Another change of variable $\left(t_{d}=x_{d}+c_{1}\left(\boldsymbol{x}^{\prime}\right) / n, \boldsymbol{t}^{\prime}=\boldsymbol{x}^{\prime}\right)$ eliminates $c_{1}\left(\boldsymbol{x}^{\prime}\right)$, so we consider only the case $c_{1}\left(\boldsymbol{x}^{\prime}\right) \equiv 0$. Let $\mathcal{M}$ denote the set of $j \in\{2, \ldots, n\}$ such that $c_{j} \neq 0$ which we assume to be non-empty.

Now apply the induction hypothesis to the product

$$
\prod_{k \in \mathcal{M}} c_{k}\left(\boldsymbol{x}^{\prime}\right) \prod_{i, j \in \mathcal{M}, i<j}\left(c_{i}\left(\boldsymbol{x}^{\prime}\right)^{n ! / i}-c_{j}\left(\boldsymbol{x}^{\prime}\right)^{n ! / j}\right)
$$

By a sequence of right compositions with diffeomorphisms, blow-ups, and ramifications, we arrive at a situation where the product has normal crossings. Thus every $c_{j}\left(\boldsymbol{x}^{\prime}\right)$ and every $c_{k}\left(\boldsymbol{x}^{\prime}\right)^{d ! / i}-c_{j}\left(\boldsymbol{x}^{\prime}\right)^{d ! / j}$ in the product has normal crossings. It is easy to deduce (see Lemma 4.7 in $[\mathbf{B M}])$ that if $c_{j}\left(\boldsymbol{x}^{\prime}\right)=\boldsymbol{x}^{\prime \gamma_{j}} U_{j}\left(\boldsymbol{x}^{\prime}\right)$ with $U_{j}(\mathbf{0}) \neq 0$, then the set $\left\{\frac{n !}{j} \gamma_{j}\right.$ $j \in \mathcal{M}\}$ is totally ordered with respect to the relation $\preceq$ defined by $\alpha \preceq \beta$ if $\alpha_{i} \leq \beta_{i}$ for all $i$. A ramification in the first $d-1$ variables allows us to suppose that, moreover, $\gamma_{j}$ is divisible by $j$ for all $j \in \mathcal{M}$ and thus the subset $\left\{\frac{1}{j} \gamma_{j} \mid j \in \mathcal{M}\right\}$ of $\mathbb{N}^{d-1}$ is totally ordered. Let $l \in \mathcal{M}$ be such $\frac{1}{l} \gamma_{l} \leq \frac{1}{j} \gamma_{j}$ for every $j \in \mathcal{M}$.

Denote $\gamma_{j}=\left(\gamma_{j 1}, \ldots, \gamma_{j, d-1}\right), j \in \mathcal{M}$. For the above $l$, let $k$ be the largest index such that $\gamma_{l k} \neq 0$ (and consequently, $\gamma_{j k} / j \geq \gamma_{l k} / l \geq 1$ for every $j \in \mathcal{M}$ ).

Now, blow-up with center $x_{k}=x_{d}=0$. For $\xi=\infty$, this means replacing $x_{k}$ by $x_{k} x_{d}$. Then for $j \in \mathcal{M}$, the term $\boldsymbol{x}^{\prime \gamma_{j}} x_{d}^{n-j}$ is transformed into $\boldsymbol{x}^{\prime \gamma_{j}} x_{d}^{\gamma_{j k}+n-j}$ which can be divided by $x_{d}^{n}$, because $\gamma_{j k} \geq j$. Hence $f$ is transformed into $\tilde{f}(\boldsymbol{x})=x_{d}^{n}\left(1+\sum_{j \in \mathcal{M}} \boldsymbol{x}^{\prime \gamma_{j}} q_{j}(\boldsymbol{x}) x_{d}^{\gamma_{j k}-j}\right)$ with some $q_{j}$ analytic at the origin. Clearly, $\tilde{f}$ has normal crossings.

For $\xi \in \mathbb{C}$, this blow-up means to replace $x_{d}$ by $x_{k}\left(x_{d}+\xi\right)$. Then for $j \in \mathcal{M}$, the term $\boldsymbol{x}^{\prime \gamma_{j}} x_{d}^{n-j}$ is transformed into

$$
\left(\prod_{i \neq k, d} x_{i}^{\gamma_{j i}}\right) x_{k}^{\gamma_{j k}+n-j}\left(x_{d}+\xi\right)^{n-j},
$$


which can be divided by $x_{k}^{n}$, because $\gamma_{j k} \geq j$. Thus $f(\boldsymbol{x})$ is transformed into

$$
\tilde{f}(\boldsymbol{x})=x_{k}^{n}\left(\left(\xi+x_{d}\right)^{n}+\tilde{c}_{2}\left(\boldsymbol{x}^{\prime}\right)\left(\xi+x_{d}\right)^{n-2}+\cdots+\tilde{c}_{n}\left(\boldsymbol{x}^{\prime}\right)\right),
$$

where $\tilde{c}_{j}\left(\boldsymbol{x}^{\prime}\right)=x_{k}^{-j} c_{j}\left(\boldsymbol{x}^{\prime}\right)$ is analytic at the origin. We continue then with the second factor $\bar{f}(\boldsymbol{x})=x_{k}^{-n} \tilde{f}(\boldsymbol{x})$.

If all $\tilde{c}_{j}(\mathbf{0})$ vanish, we have normal crossings for $\xi \neq 0$, but have to blow-up another time if $\xi=0$ (we do not use ramifications as $\gamma_{j}$ is already divisible by $j$ for $j \in \mathcal{M})$. After a finite number of such blowups, at least one of the $\tilde{c}_{j}(\mathbf{0}) \neq 0$. The second factor $\bar{f}(\boldsymbol{x})=x_{k}^{-n} \tilde{f}(\boldsymbol{x})$ is then of lower order than $f$ for all $\xi$ and also $x_{d}$-regular. Indeed, $\bar{f}\left(0, \ldots, 0, x_{d}\right)=\left(\xi+x_{d}\right)^{n}+\tilde{c}_{2}(\mathbf{0})\left(\xi+x_{d}\right)^{n-2}+\cdots+\tilde{c}_{n}(\mathbf{0})$ might vanish at $x_{d}=0$, but because it has no term with $\left(\xi+x_{d}\right)^{n-1}$, it cannot be equal to $x_{d}^{n}$ and hence cannot vanish of order $n$. As $\bar{f}\left(0, \ldots, 0, x_{d}\right)$ contains some term $x_{d}^{m}, m<n$, this term is also in $\bar{f}$ and hence it is at most of order $m$.

A first application of this monomialization lemma is the following statement. It may be well-known to specialists, but lacking precise references we include a proof for the sake of completeness.

Lemma 2.2. Let $f, g \in \mathcal{O}, f(\mathbf{0})=g(\mathbf{0})=0, g \neq 0$ be germs of holomorphic functions. Assume that both are defined on $D(\mathbf{0} ; r)$ and that $\left|\frac{f(\boldsymbol{x})}{g(\boldsymbol{x})}\right|$ is bounded on the set $A_{\theta, r}$ of all $\boldsymbol{x} \in D(\mathbf{0} ; r)$ such that $g(\boldsymbol{x}) \neq 0$ and $\arg g(\boldsymbol{x})=\theta$. Then $g$ divides $f$, i.e. there exists $q \in \mathcal{O}$ such that $f=q g$.

Proof: Note first that this result is much easier in dimension one. Indeed, consider functions $f, g$ holomorphic in some neighbourhood of $0 \in \mathbb{C}$ such that $f(0)=g(0)=0, g \neq 0$. Then the quotient $f / g$ has at most a pole at 0 , if we restrict ourselves to a small enough neighbourhood of the origin. If there exist a sequence $\left\{t_{n}\right\}_{n=1}^{\infty}$ such that $\lim _{n \rightarrow \infty} t_{n}=0$ for which $\left|f\left(t_{n}\right) / g\left(t_{n}\right)\right|$ is bounded, the origin cannot be a pole, so it is a removable singularity: there exists a germ $q \in \mathcal{O}$ such that $f=g q$.

In arbitrary dimension, we proceed by induction on $h(f g)$. At some points it might be necessary to reduce $r$ but we do not always mention this. If $h(f g)=0, f$ and $g$ have normal crossings and we can assume that

$$
f(\boldsymbol{x})=x_{1}^{m_{1}} \cdots x_{d}^{m_{d}} U_{1}(\boldsymbol{x}) ; \quad g(\boldsymbol{x})=x_{1}^{\ell_{1}} \cdots x_{d}^{\ell_{d}} U_{2}(\boldsymbol{x})
$$

with germs $U_{1}$ and $U_{2}, U_{1}(\mathbf{0}) U_{2}(\mathbf{0}) \neq 0$. If all $\ell_{j}$ vanish, there is nothing to show. Otherwise, we can assume that $\ell_{1}>0$. 
For fixed $x_{2}, \ldots, x_{d} \neq 0$ and if $r$ is sufficiently small, we can apply the one-dimensional result with respect to the variable $x_{1}$ and appropriate $\theta_{1}$. This implies that $m_{1} \geq \ell_{1}$. Similarly, we obtain that $m_{j} \geq \ell_{j}$ for all $j$ and the statement follows.

Assume now that the statement is true for all couples $(f, g)$ with $h(f g) \leq m$ for some $m \in \mathbb{N}$. Consider some couple $(f, g)$ satisfying the assumptions of the lemma and $h(f g)=m+1$. As the statement is stable with respect to right composition by diffeomorphisms, we can assume that $h\left(\left(f \circ b_{\xi}\right)\left(g \circ b_{\xi}\right)\right) \leq m$ for all $\xi \in \mathbb{P}_{\mathbb{C}}^{1}$ or that there is a $k \in \mathbb{N}$ with $h\left(\left(f \circ r_{k}\right)\left(g \circ r_{k}\right)\right) \leq m$. In the latter case, the assumption of the lemma implies that $f\left(r_{k}(\boldsymbol{t})\right) / g\left(r_{k}(\boldsymbol{t})\right)$ is bounded on the set of all sufficiently small $\boldsymbol{t} \in \mathbb{C}^{d}$ such that $g\left(r_{k}(\boldsymbol{t})\right) \neq 0$ and $\arg \left(g\left(r_{k}(\boldsymbol{t})\right)\right)=\theta$. Hence there exists $q \in \mathcal{O}$ such that

$$
f \circ r_{k}=\left(g \circ r_{k}\right) q
$$

As $f \circ r_{k}$ and $g \circ r_{k}$ are invariant under the rotation $\boldsymbol{t} \mapsto\left(e^{2 \pi i / k} t_{1}, \boldsymbol{t}^{\prime}\right)$, so is $q$ and hence there is a germ $Q \in \mathcal{O}$ such that $q=Q \circ r_{k}$. We obtain that $f=g Q$ and the statement follows.

Consider now the first case that $h\left(\left(f \circ b_{\xi}\right)\left(g \circ b_{\xi}\right)\right) \leq m$ for all $\xi \in \mathbb{P}_{\mathbb{C}}^{1}$. The assumption of the lemma implies that $f\left(b_{\xi}(\boldsymbol{t})\right) / g\left(b_{\xi}(\boldsymbol{t})\right)$ is bounded on the set of all sufficiently small $\boldsymbol{t} \in \mathbb{C}^{d}$ such that $g\left(b_{\xi}(\boldsymbol{t})\right) \neq 0$ and $\arg \left(g\left(b_{\xi}(\boldsymbol{t})\right)\right)=\theta$. Hence for every $\xi \in \mathbb{P}_{\mathbb{C}}^{1}$ there exists $q_{\xi} \in \mathcal{O}$ such that

$$
f \circ b_{\xi}=\left(g \circ b_{\xi}\right) q_{\xi} .
$$

Thus for every $\xi \in \mathbb{P}_{\mathbb{C}}^{1}$, there is an open neighborhood $W_{\xi}$ of $\mathbf{0}$, where $f \circ b_{\xi}, g \circ b_{\xi}$, and $q_{\xi}$ are defined. With these we consider the open neighborhoods $U_{\xi}=\phi_{\xi}^{-1}\left(W_{\xi}\right)$ of $(\xi, \mathbf{0}) \in M$ (see Subsection 2.2 for notation) and the holomorphic $Q_{\xi}: U_{\xi} \rightarrow \mathbb{C}$ defined by $Q_{\xi}=q_{\xi} \circ \phi_{\xi}$. By definition, for $\xi, \zeta \in \mathbb{P}_{\mathbb{C}}^{1}$, we have $Q_{\xi}(p)=Q_{\zeta}(p)$ for $p \in U_{\xi} \cap U_{\zeta}$ with $g(b(p)) \neq 0$. As $g$ is not identically zero, this means that $Q_{\xi}$ and $Q_{\zeta}$ coincide on an open and dense subset of $U_{\xi} \cap U_{\zeta}$. Therefore $Q_{\xi}=Q_{\zeta}$ on this intersection and thus all $Q_{\xi}, \xi \in \mathbb{P}_{\mathbb{C}}^{1}$ define a holomorphic function $Q: U \rightarrow \mathbb{C}$, where $U$ is some neighbourhood of $\mathbb{P}_{\mathbb{C}}^{1} \times\{\mathbf{0}\} \subseteq M . \mathbb{P}_{\mathbb{C}}^{1}$ being compact, $Q$ is constant over it, so there exists a holomorphic $q: V \rightarrow \mathbb{C}$, $V$ a neighbourhood of $\mathbf{0} \in \mathbb{C}^{d}$, such that $q \circ b=Q$ (apply Hartogs' theorem). By construction, we have $f \circ b_{\xi}=\left(g \circ b_{\xi}\right)\left(q \circ b_{\xi}\right)$ for all $\xi \in \mathbb{P}_{\mathbb{C}}^{1}$ and thus we obtain that $f=g q$, as desired.

Lemma 2.2 is basic for our article, but especially for the study of Gevrey asymptotics with respect to an analytic function, we need more quantitative information about division. 
Lemma 2.3. Let $D^{\prime}, D$ denote two open connected subsets of $\mathbb{C}^{d}$ such that the closure of $D^{\prime}$ is contained in $D$ and compact. Suppose that $P$ is holomorphic in $D$ and not identically vanishing. Let $\theta \in \mathbb{R}$ and $A_{\theta, D}=\{\boldsymbol{x} \in D \mid P(\boldsymbol{x}) \neq 0, \arg (P(\boldsymbol{x}))=\theta\}$. For $n \in \mathbb{N}$ let $M_{n}$ denote the set of all functions $f \in \mathcal{O}_{b}(D)$ such that $f / P^{n}$ is bounded on $A_{\theta, D}$.

Then $f / P^{n}$ can be analytically continued to $D^{\prime}$ if $f \in M_{n}$. Furthermore, there is a constant $K>0$ such that the mappings $q_{n}: M_{n} \rightarrow$ $\mathcal{O}_{b}\left(D^{\prime}\right)$ associating to $f \in M_{n}$ the analytic continuation of $f / P^{n}$ to $D^{\prime}$ have norms $\leq K^{n}$ (provided $M_{n}$ and $\mathcal{O}_{b}\left(D^{\prime}\right)$ are equipped with the maximum norm).

Proof: The first statement is proved by applying Lemma 2.2 in the neighborhood of each point of $D^{\prime}$.

For the second statement, it is sufficient to prove the existence of such a constant in some neighborhood of every point of the closure of $D^{\prime}$ and then use the compactness of the latter.

In order to show it in some neighborhood of some point $\boldsymbol{x}_{0}$ of the closure of $D^{\prime}$, one uses induction on $h\left(P \circ T_{x_{0}}\right), T_{x_{0}}$ the translation $\boldsymbol{x} \mapsto$ $\boldsymbol{x}+\boldsymbol{x}_{0}$, similarly to the proof of Lemma 2.2. If $P\left(\boldsymbol{x}_{0}\right) \neq 0$, the statement is trivial, if $P \circ T_{x_{0}}$ has normal crossings, the existence of such a constant follows by Schwarz's lemma. Indeed, assume that $D\left(\boldsymbol{x}_{0}, \boldsymbol{r}\right) \subset D^{\prime}$ and that $\left(P \circ T_{x_{0}}\right)(\boldsymbol{x})=\boldsymbol{x}^{\boldsymbol{\alpha}} U(\boldsymbol{x}), U$ a unit, i.e. $U(\mathbf{0}) \neq 0$, and without loss of generality (reduce $\boldsymbol{r}$ otherwise) there is a constant $\mu>0$ such that $|U(\boldsymbol{x})| \geq \mu$ for $\boldsymbol{x} \in D\left(\boldsymbol{x}_{0}, \boldsymbol{r}\right)$. Given $f \in M_{n}$, we can apply Schwarz's lemma repeatedly to $f / U^{n}$ and obtain that

$$
\sup _{\boldsymbol{x} \in D\left(\boldsymbol{x}_{0}, \boldsymbol{r}\right)}\left|q_{n}(f)(\boldsymbol{x})\right| \leq \sup _{\boldsymbol{x} \in D\left(\boldsymbol{x}_{0}, \boldsymbol{r}\right)}|f(\boldsymbol{x})| \boldsymbol{r}^{-n \boldsymbol{\alpha}} \mu^{-n} .
$$

Hence $K=\boldsymbol{r}^{-\boldsymbol{\alpha}} \mu^{-1}$ satisfies the wanted estimates in $D\left(\boldsymbol{x}_{0}, \boldsymbol{r}\right)$.

If $h\left(P \circ T_{x_{0}}\right)=m>0$, we can assume that either $h\left(P \circ T_{x_{0}} \circ b_{\xi}\right)<m$ for all $\xi \in \mathbb{P}_{\mathbb{C}}^{1}$ or there is a $k \in \mathbb{N}$ such that $h\left(P \circ T_{x_{0}} \circ r_{k}\right)<m$. In the former case there exists such a constant $K_{\xi}$ and a neighborhood $V_{\xi}$ of the origin for $P \circ T_{x_{0}} \circ b_{\xi} ; \xi \in \mathbb{P}_{\mathbb{C}}^{1}$ arbitrary. Using the compactness of $\mathbb{P}_{\mathbb{C}}^{1}$ as in the previous proof, the existence of such a constant $K$ for $P$ and some neighborhood of $\mathbf{0}$ follows.

In the latter case $\left(h\left(P \circ T_{x_{0}} \circ r_{k}\right)<m\right)$, we can use the same constant before and after ramification and only adjust the neighborhood.

2.3. Generalized Weierstrass Division. We will present here a version adapted and simplified by Stevens [Ste] of a generalized Weierstrass Division theorem, who attributes it to Galligo [Gal], but whose original version is due to Aroca, Hironaka, and Vicente [AHV]. The version (see Lemma 2.6) for functions bounded and holomorphic on certain special neighborhoods of the origin is particularly useful in the sequel. 
Let $S$ be either the ring $\mathcal{O}=\mathbb{C}\{\boldsymbol{x}\}=\mathbb{C}\left\{x_{1}, x_{2}, \ldots, x_{d}\right\}$ or $\hat{\mathcal{O}}=\mathcal{R} \llbracket \boldsymbol{x} \rrbracket$ where $\mathcal{R}$ is an integral domain; in most cases we will use $\mathcal{R}=\mathbb{C}$. Each $f \in$ $S$ can be written as a (formal) power series

$$
f=\sum_{\alpha \in \mathbb{N}^{d}} f_{\alpha} x^{\alpha},
$$

where the monomials $\boldsymbol{x}^{\boldsymbol{\alpha}}=x_{1}^{\alpha_{1}} \cdots x_{d}^{\alpha_{d}}$ are defined as usual. Let $\ell: \mathbb{N}^{d} \rightarrow$ $\mathbb{R}_{+}$be an injective linear form, $\ell(\boldsymbol{\alpha})=\ell_{1} \alpha_{1}+\cdots+\ell_{d} \alpha_{d}$. As in [Ste], we define a total ordering on the monomials by

$$
\boldsymbol{x}^{\boldsymbol{\alpha}}<_{\ell} \boldsymbol{x}^{\boldsymbol{\beta}} \text { if and only if } \ell(\boldsymbol{\alpha})<\ell(\boldsymbol{\beta}) .
$$

For $f \in S \backslash\{0\}$ written according to (2.3), we will say that $\boldsymbol{\alpha}$ is the minimal exponent of $f$, and we will denote $v_{\ell}(f)=\boldsymbol{\alpha}$ if

$$
\boldsymbol{x}^{\boldsymbol{\alpha}}=\min \left\{\boldsymbol{x}^{\boldsymbol{\beta}} \mid \boldsymbol{\beta} \in \mathbb{N}^{d} ; f_{\boldsymbol{\beta}} \neq 0\right\},
$$

where the minimum is taken according to the ordering $<_{\ell}$. Observe that $v_{\ell}$ is compatible with the multiplication: $v_{\ell}(f g)=v_{\ell}(f)+v_{\ell}(g)$ if $f, g \neq 0$. Therefore the multiples of some nonzero $f \in S$ (i.e. the elements of $f S$ ) have minimal exponents in $v_{\ell}(f)+\mathbb{N}^{d}$. The converse is false in general, of course.

Given a nonzero $P \in S$ with $v_{\ell}(P)>_{\ell} \mathbf{0}$, we introduce the set

$$
\Delta_{\ell}(P)=\left\{g=\sum g_{\boldsymbol{\alpha}} x^{\boldsymbol{\alpha}} ; g_{\boldsymbol{\alpha}}=0 \text { if } \boldsymbol{\alpha} \in v_{\ell}(P)+\mathbb{N}^{d}\right\} .
$$

In the case of two variables and $v_{\ell}(P)=\left(a_{1}, a_{2}\right), a_{1}, a_{2}>0$, this set can be written as

$$
\bigoplus_{j=0}^{a_{1}-1} x_{1}^{j} \mathbb{C} \llbracket x_{2} \rrbracket \oplus x_{1}^{a_{1}} \bigoplus_{k=0}^{a_{2}-1} x_{2}^{k} \mathbb{C} \llbracket x_{1} \rrbracket .
$$

In the general case it is possible to express $\Delta_{\ell}(P)$ in a similar way, but we do not write down this cumbersome formula. In the rest of Subsection 2.3 we omit the index $\ell$ for the sake of simplicity.

Lemma 2.4. Let $P \in S, P \neq 0$ with $\ell(v(P))>0$ and let $\Delta(P)$ be defined by (2.4). In the case $S=\mathcal{R} \llbracket \boldsymbol{x} \rrbracket$, we assume that the coefficient $P_{v(P)}$ of $\boldsymbol{x}^{v(P)}$ in $P$ is a unit in $\mathcal{R}$. Then for every $g \in S$, there exist unique $q \in S$ and $r \in \Delta(P)$ such that

$$
g=q P+r .
$$

Proof in the case $S=\mathbb{C}\{\boldsymbol{x}\}$ : We even prove it in the case of the Banach space $\mathcal{S}_{\mu}=\mathcal{O}_{b}\left(D_{\mu}\right)$, with norm $\|\cdot\|_{\infty}$, where $D_{\mu}=D\left(\mathbf{0} ;\left(\mu^{\ell_{1}}, \ldots, \mu^{\ell_{d}}\right)\right)$, if $\mu>0$ is sufficiently small. The set $D_{\mu}$ has been chosen so that $\left|x^{\boldsymbol{\alpha}}\right| \leq \mu^{\ell(\boldsymbol{\alpha})}$ for $x \in D_{\mu}$. 
If we define

$$
T_{j} g(\boldsymbol{x})= \begin{cases}\left(g(\boldsymbol{x})-g\left(x_{1}, \ldots, x_{j-1}, 0, x_{j+1}, \ldots, x_{d}\right)\right) / x_{j} & \text { if } x_{j} \neq 0, \\ \frac{\partial g}{\partial x_{j}}(\boldsymbol{x}) & \text { if } x_{j}=0,\end{cases}
$$

then with $g \in \mathcal{S}_{\mu}$, also $T_{j} g \in \mathcal{S}_{\mu}$ and $\left\|T_{j} g\right\|_{\infty} \leq 2 \mu^{-\ell_{j}}\|g\|_{\infty}$.

We put $\boldsymbol{a}=v(P)$. Clearly, every $g \in \mathcal{S}_{\mu}$ can be written uniquely

$$
g=Q_{0}(g) \boldsymbol{x}^{\boldsymbol{a}}+R_{0}(g) \quad \text { where } Q_{0}(g) \in \mathcal{O}, R_{0}(g) \in \Delta(P) \cap \mathcal{O} .
$$

Rewriting $Q_{0}(g)=T_{1}^{a_{1}} \cdots T_{d}^{a_{d}} g$ we find that $Q_{0}$ is a linear operator from $\mathcal{S}_{\mu}$ to itself and satisfies $\left\|Q_{0}(g)\right\|_{\infty} \leq 2^{|\boldsymbol{a}|} \mu^{-\ell(\boldsymbol{a})}\|g\|_{\infty}$ for all $g \in \mathcal{S}_{\mu}$.

We can suppose $P_{\boldsymbol{a}}=1$ without loss of generality. Then $P=\boldsymbol{x}^{\boldsymbol{a}}+\tilde{P}$ with some $\tilde{P} \in \mathcal{S}_{\mu}, v(\tilde{P})>_{\ell} \boldsymbol{a}$. We can rewrite equation (2.5) as $q \boldsymbol{x}^{\boldsymbol{a}}+$ $r=g-\tilde{P} q$ which is equivalent to the fixed point equation

$$
q=Q_{0}(g-\tilde{P} q)
$$

together with $r=R_{0}(g-\tilde{P} q)$. We can find a constant $K>0$ such that $\|\tilde{P}\|_{\infty} \leq K \mu^{\ell(v(\tilde{P}))}$ if $\mu$ is sufficiently small. This finally yields

$$
\left\|Q_{0}(\tilde{P} h)\right\|_{\infty} \leq K 2^{|\boldsymbol{a}|} \mu^{\ell(v(\tilde{P}))-\ell(\boldsymbol{a})}\|h\|_{\infty}
$$

for all $h \in \mathcal{S}_{\mu}, \mu$ sufficiently small. Therefore the right hand side of (2.7) defines a contraction on $\mathcal{S}_{\mu}$ and hence it has a unique solution if $\mu>0$ is sufficiently small. As (2.7) together with $r=R_{0}(g-\tilde{P} q)$ is equivalent to (2.5), this implies the statement of the lemma in the case of $S=\mathcal{S}_{\mu}$ and also in the case of $S=\mathcal{O}=\mathbb{C}\{\boldsymbol{x}\}$.

Concerning the proof in the case of $S=\hat{\mathcal{O}}=\mathbb{C} \llbracket \boldsymbol{x} \rrbracket$, we define $w(f)=$ $\ell(v(f))$ for $f \in S$. Then $w$ is a discrete valuation on $S: w(f+g) \geq$ $\min (w(f), w(g))$ and $w(f g)=w(f)+w(g)$, and $\delta(g, h)=2^{-w(g-h)}$ makes $S$ into a complete metric space. In the same way as above, equation (2.5) of the lemma is equivalent to the fixed point equation (2.7) and it can be shown that it has exactly one solution.

An immediate consequence of the above lemma is

Corollary 2.5. Under the assumptions of Lemma 2.4, every $f \in \mathcal{R} \llbracket \boldsymbol{x} \rrbracket$ can be written uniquely in the form

$$
\hat{f}=\sum_{n=0}^{\infty} g_{n} P^{n}
$$

where $g_{n} \in \Delta(P)$ for all $n \in \mathbb{N}$.

Proof: Using Lemma 2.4 repeatedly, we can write (uniquely)

$$
\hat{f}=g_{0}+g_{1} P+g_{2} P^{2}+\cdots+g_{N-1} P^{N-1}+q_{N} P^{N},
$$

where $N \in \mathbb{N}$, all $g_{n} \in \Delta(P)$, and $q_{N} \in \mathcal{R} \llbracket \boldsymbol{x} \rrbracket$. As $N \rightarrow \infty$, the statement follows by $\mathfrak{m}$-adic convergence. 
For later use, we note the statement proved in the first part of the proof of Lemma 2.4 and prove the analogue of Corollary 2.5 for $\mathbb{C}\{\boldsymbol{x}\}$.

Lemma 2.6. Let $\ell, P$ be as above Lemma 2.4 and let $\Delta(P)$ be defined by (2.4). For $s>0$ let $D_{s}=D\left(\mathbf{0} ;\left(s^{\ell_{1}}, \ldots, s^{\ell_{d}}\right)\right)$. If $s$ is sufficiently small, then for every $g \in \mathcal{O}_{b}\left(D_{s}\right)$ there exist unique $r \in \mathcal{O}_{b}\left(D_{s}\right)$ with $J(r) \in \Delta(P)$ and $q \in \mathcal{O}_{b}\left(D_{s}\right)$ such that $g=q P+r$.

The corresponding operators $Q, R: \mathcal{O}_{b}\left(D_{s}\right) \rightarrow \mathcal{O}_{b}\left(D_{s}\right)$ defined by $g \mapsto$ $q$ (respectively $g \mapsto r$ ) are linear and continuous.

Corollary 2.7. Under the assumptions of Lemma 2.4, for every $f \in$ $\mathbb{C}\{\boldsymbol{x}\}$ there exist $\rho>0$ and a sequence $\left\{g_{n}\right\}_{n \in \mathbb{N}}$ in $\mathcal{O}_{b}(D(\mathbf{0} ; \rho))$ with $J\left(g_{n}\right) \in \Delta(P)$ for all $n$ such that $f$ can be written in the form

$$
f(\boldsymbol{x})=\sum_{n=0}^{\infty} g_{n}(\boldsymbol{x}) P(\boldsymbol{x})^{n} \quad \text { for }|\boldsymbol{x}| \leq \rho .
$$

The functions $g_{n}$ are uniquely determined by Corollary 2.5.

Proof: For $s>0$ sufficiently small, $f \in \mathcal{O}_{b}\left(D_{s}\right)$ and the operators $Q, R$ of the preceding lemma are defined on $\mathcal{O}\left(D_{s}\right)$. For $N \in \mathbb{N}$, we obtain

$$
f(\boldsymbol{x})=\sum_{n=0}^{N-1}\left(\left(R Q^{n}\right) f\right)(\boldsymbol{x}) P(\boldsymbol{x})^{n}+\left(Q^{N} f\right)(\boldsymbol{x}) P(\boldsymbol{x})^{N}
$$

by repeated application of Lemma 2.6 .

If $\rho \in] 0, s]$ is so small that $M:=\sup \{|P(\boldsymbol{x})| \mid \boldsymbol{x} \in D(\mathbf{0}, \rho)\}<\frac{1}{\|Q\|}$ then we can estimate

$$
\sup _{|\boldsymbol{x}|<\rho}\left|f(\boldsymbol{x})-\sum_{n=0}^{N-1}\left[\left(\left(R Q^{n}\right) f\right) P^{n}\right](\boldsymbol{x})\right| \leq(M\|Q\|)^{N} \sup _{\mathbf{y} \in D_{s}}|f(\mathbf{y})| .
$$

This proves the statement.

2.4. A Cousin problem. In the sequel we use the following lemma solving a certain Cousin problem for $\mathbb{P}_{\mathbb{C}}^{1}$. Let $I$ denote some finite set, $\infty \in I, \mathcal{U}=\left(U_{i}\right)_{i \in I}$ a finite cover of $\mathbb{P}_{\mathbb{C}}^{1}$ by open sets. Assume $\infty \in$ $U_{\infty}$ for simplicity of notation. Let $\left(U_{i j}\right)_{i, j \in I}$ denote the collection of intersections $U_{i j}=U_{i} \cap U_{j}$.

Lemma 2.8. Let $\mathcal{C}^{0}(\mathcal{U})$ denote the Banach space of collections $f=$ $\left(f_{i}\right)_{i \in I}$ of bounded holomorphic $f_{i}: U_{i} \rightarrow \mathbb{C}$ such that $f_{\infty}(\infty)=0$, equipped with the maximum norm. Let $\mathcal{Z}^{1}(\mathcal{U})$ denote the Banach space of collections $d=\left(d_{i j}\right)_{i, j \in I}$ of bounded holomorphic $d_{i j}: U_{i j} \rightarrow \mathbb{C}$ satisfying the cocycle condition

$$
d_{i j}(z)+d_{j k}(z)=d_{i k}(z), \quad \text { if } z \in U_{i} \cap U_{j} \cap U_{k},
$$

equipped with the maximum norm. 
Then the boundary mapping $\delta: \mathcal{C}^{0}(\mathcal{U}) \rightarrow \mathcal{Z}^{1}(\mathcal{U})$ defined by

$$
\delta\left(\left(f_{i}\right)_{i \in I}\right)=\left(f_{i}-f_{j}\right)_{i, j \in I}
$$

is bijective, linear, and continuous and its inverse, denoted by $\Sigma$, is also continuous.

Proof: $\delta$ is surjective. Since $H^{1}(\mathcal{U}, \mathcal{O})=0$ as is well known, there exist, for every $\left(d_{i j}\right)_{i, j \in I} \in \mathcal{Z}^{1}(\mathcal{U})$, a family $\left(f_{i}\right)_{i \in I}, f_{i}: U_{i} \rightarrow \mathbb{C}$ holomorphic, such that $d_{i j}=f_{i}-f_{j}$ for $i, j \in I$. The additional condition $f_{\infty}(\infty)=0$ is achieved by adding the same suitable constant to each $f_{i}$. It remains to show that each $f_{i}$ is bounded.

As $\mathcal{U}$ is a cover of $\mathbb{P}_{\mathbb{C}}^{1}$, each point $c \in \mathbb{P}_{\mathbb{C}}^{1}$ is contained in some $U_{i(c)}$; hence there exists a neighborhood $V_{c}$ of $c$ the closure of which is contained in $U_{i(c)}$. Therefore $f_{i(c)}$ is bounded on $V_{c}$. For $j \in I, j \neq i(c)$, the function $f_{j}$ can be written $f_{j}=f_{i(c)}+d_{j i(c)}$ on $V_{c} \cap U_{j} \subset U_{i(c) j}$, provided that $U_{i(c) j} \neq \emptyset$, and therefore $f_{j}$ is also bounded on $V_{c} \cap U_{j}$ as $d_{i(c) j}$ and $f_{i(c)}$ are. We have shown that every $c \in \mathbb{P}_{\mathbb{C}}^{1}$ has a neighborhood $V_{c}$ such that for all $j \in I$, the function $f_{j}$ is bounded on $V_{c} \cap U_{j}$. By the compactness of $\mathbb{P}_{\mathbb{C}}^{1}$, a finite number of such neighborhoods $V_{c}$ covers $\mathbb{P}_{\mathbb{C}}^{1}$ and the boundedness of all $f_{j}, j \in I$, follows. This completes the proof that $\delta$ is surjective.

$\delta$ is injective, because its kernel is $\{0\}$. Indeed, if $\delta\left(\left(f_{i}\right)_{i \in I}\right)=(0)_{i, j \in I}$, then $f_{i}(z)=f_{j}(z)$ whenever $z \in U_{i} \cap U_{j}$. Hence $\left(f_{i}\right)_{i \in I}$ is actually the collection of restrictions of some analytic function $f: \mathbb{P}_{\mathbb{C}}^{1} \rightarrow \mathbb{C}$ to the $\left(U_{i}\right)_{i \in I}$. By Liouville's theorem, $f$ is then a constant. The condition $f_{\infty}(\infty)=0$ now implies that $f=0$; hence $\left(f_{i}\right)_{i \in I}=0$.

Obviously $\delta$ is linear and continuous. Therefore, by the theorem of the bounded inverse, its inverse is also continuous.

In the sequel, we need an extension of the above lemma to functions depending holomorphically upon parameters.

Lemma 2.9. Consider a collection $\left(d_{i j}\right)_{i, j \in I}$ of functions holomorphic on $B \times U_{i j}$, where $B$ is some open subset of $\mathbb{C}^{m}, m \geq 1$, satisfying the cocycle condition with respect to the second variable. There exists a collection $\left(f_{i}\right)_{i \in I}$ of holomorphic functions on $B \times U_{i}, i \in I$, such that $f_{i}(b, t)-f_{j}(b, t)=d_{i j}(b, t)$ for all $b \in B$ and $t \in U_{i j}$.

If there exists a function $K: B \rightarrow \mathbb{R}_{+}$such that for every $b \in B$, the collection of functions $\left(z \mapsto d_{i j}(b, z)\right)_{i, j \in I}$ is bounded by $K(b)$, then the collection of functions $\left(z \mapsto f_{i}(b, z)\right)_{i \in I}$ is bounded by $\|\Sigma\| K(b)$ for every $b \in B$. 
Proof: We define the collection $\left(f_{i}\right)_{i \in I}$ by $f_{i}(b, z)=\phi_{i}^{b}(z)$, where

$$
\left(\phi_{i}^{b}\right)_{i \in I}=\Sigma\left(\left(z \mapsto d_{i j}(b, z)\right)_{i, j \in I}\right) \text { for } b \in B,
$$

where $\Sigma$ is the operator of Lemma 2.8. Then the second statement of our lemma follows from Lemma 2.8. It is not clear, however, that the functions $f_{i}$ are holomorphic with repect to both variables.

In order to prove this, we first choose an open cover $V_{i}, i \in I$, of $\mathbb{P}_{\mathbb{C}}^{1}$ such that for every $i$, the $\operatorname{closure} \operatorname{cl}\left(V_{i}\right)$ is a subset of $U_{i}$. The set $V_{\infty}$ can be chosen such that, additionally, it contains $\infty$. Let $\tilde{\Sigma}$ denote the operator of Lemma 2.8 applied to the cover $\mathcal{V}=\left(V_{i}\right)_{i \in I}$.

Now fix any $b_{0} \in B$ and choose $\rho>0$ such that $\operatorname{cl}\left(D\left(b_{0}, \rho\right)\right) \subset$ $B$. Then the collection $\left(\tilde{d}_{i j}\right)_{i, j \in I}, \tilde{d}_{i j}=\left.d_{i j}\right|_{D\left(b_{0}, \rho\right) \times\left(V_{i} \cap V_{j}\right)}$, consists of bounded holomorphic functions. Then we use that for all open subsets $D \subset \mathbb{C}^{m}$ the Banach space $\mathcal{O}_{b}(D \times \mathcal{V}, \mathbb{C})$ is canonically isometrically isomorphic to $\mathcal{O}_{b}\left(D, \mathcal{O}_{b}(\mathcal{V}, \mathbb{C})\right)$ and that Cauchy's formula with respect to the first variable commutes with the continuous linear operator $\tilde{\Sigma}$, applied with respect to the second variable. We obtain that the collection $\left(\tilde{f}_{i}\right)_{i \in I}$ defined by $\tilde{f}_{i}(b, z)=\psi_{i}^{b}(z)$,

$$
\left(\psi_{i}^{b}\right)_{i \in I}=\tilde{\Sigma}\left(\left(z \mapsto \tilde{d}_{i j}(b, z)\right)_{i, j \in I}\right) \quad \text { for } b \in D\left(b_{0}, \rho\right),
$$

consists of bounded holomorphic functions on $D\left(b_{0}, \rho\right) \times V_{i}, i \in I$.

We define now a collection of holomorphic functions $\left(F_{i}\right)_{i \in I}$ on $D\left(b_{0}, \rho\right) \times U_{i}$ by $F_{i}(b, z)=\tilde{f}_{k}(b, z)+d_{i k}(b, z)$ for $b \in D\left(b_{0}, \rho\right), z \in U_{i}$ if $z \in V_{k}$ for some $k \in I$. Observe that here $k=i$ is allowed in which case $d_{i i}(b, z)=0$ and hence $F_{i}(b, z)=\tilde{f}_{i}(b, z)$. Since $V_{k}, k \in I$, form a cover of $\mathbb{P}_{\mathbb{C}}^{1}$, we can always find some $k$ such that $z \in V_{k}$. In that case $z \in U_{i} \cap V_{k} \subset U_{i k}$ and $F_{i}(b, z)$ is defined. The cocycle condition and the definition of $\tilde{f}_{k}, k \in I$, imply that the definition is independent of the choice of $k$ with $z \in V_{k}$. In a similar way, we obtain that $F_{i}(b, z)-F_{j}(b, z)=d_{i j}(b, z)$ for all $b \in D\left(b_{0}, \rho\right)$ and $z \in U_{i j}$.

As $F_{\infty}(b, \infty)=\tilde{f}_{\infty}(b, \infty)=f_{\infty}(b, \infty)=0$ for all $b \in D\left(b_{0}, \infty\right)$, we obtain that $\left(z \mapsto F_{i}(b, z)\right)_{i \in I}=\Sigma\left(\left(z \mapsto d_{i j}(b, z)\right)_{i, j \in I}\right)=\left(z \mapsto f_{i}(b, z)\right)_{i \in I}$ for all $b \in D\left(b_{0}, \rho\right)$. Therefore all functions $f_{i}$ are holomorphic with respect to $(b, z)$.

We will use a consequence of this lemma for covers of the exceptional divisor in subsequent sections. See Subsection 2.2 for notation.

Lemma 2.10. Consider an open cover $U_{j}, j=0, \ldots, N$ of the exceptional divisor $E=\mathbb{P}_{\mathbb{C}}^{1} \times\{\mathbf{0}\}$ in the blow-up variety $M$. Then there exist a positive constant $C$ and an open cover $\tilde{U}, j=0, \ldots, N$, of $E$ with $\tilde{U}_{j} \subset U_{j}, j=0, \ldots, N$, and the following property. 
Given a collection of holomorphic functions $D_{i j}: U_{i} \cap U_{j} \rightarrow \mathbb{C}, i, j=$ $0, \ldots, N$ satisfying the cocycle condition $D_{i j}(p)+D_{j k}(p)=D_{i k}(p)$ for all $i, j, k$ and $p \in U_{i} \cap U_{j} \cap U_{k}$ there exists a collection of holomorphic functions $F_{i}: \tilde{U}_{i} \rightarrow \mathbb{C}$ such that $D_{i j}(p)=F_{i}(p)-F_{j}(p)$ for all $i, j \in$ $\{0, \ldots, N\}, p \in \tilde{U}_{i} \cap \tilde{U}_{j}$, and

$$
\max _{j \in\{0, \ldots, N\}} \sup _{p \in \tilde{U}_{j}}\left|F_{j}(p)\right| \leq C \max _{i, j \in\{0, \ldots, N\}} \sup _{p \in U_{i} \cap U_{j}}\left|D_{i j}(p)\right| \leq \infty .
$$

Proof: Without loss of generality, we can assume that $(\infty, \mathbf{0}) \in U_{0}$. Then it is sufficient to prove the lemma under the additional assumption that $(\infty, \mathbf{0})$ is not an element of the other $U_{k}$.

Consider the projection $R: M \rightarrow \mathbb{P}_{\mathbb{C}}^{1} \times \mathbb{C}^{d-1}$ defined by $R((\xi, \boldsymbol{t}))=$ $\left(\xi,\left(t_{1}, \boldsymbol{t}^{\prime \prime}\right)\right)$. Its restriction to the chart $M_{0}$ is an analytic diffeomorphism onto its image. We will use the geodesic distance $d$ on $\mathbb{P}_{\mathbb{C}}^{1} \simeq S^{2}$ and denote for $\xi \in \mathbb{P}_{\mathbb{C}}^{1}, \mu>0$ by $B(\xi, \mu)$ the set of all $\zeta \in \mathbb{P}_{\mathbb{C}}^{1}$ with $d(\xi, \zeta)<\mu$. Also let $V_{i} \subset \mathbb{P}_{\mathbb{C}}^{1}$ denote the open (in $\mathbb{P}_{\mathbb{C}}^{1}$ ) set such that $V_{i} \times\{\mathbf{0}\}=U_{i} \cap E$. As in the proof of Lemma 2.9 we choose an open cover $\tilde{V}_{k}, k=0, \ldots, N$, of $\mathbb{P}_{\mathbb{C}}^{1}$ such that for every $k$, the closure $\operatorname{cl}\left(\tilde{V}_{k}\right)$ is a subset of $V_{k}$.

Fix now some $k \in\{1, \ldots, N\}$. By assumption, for all $\xi \in V_{k}$, there exists $\rho_{\xi}>0$ such that $B\left(\xi, \rho_{\xi}\right) \times D^{\prime}\left(\mathbf{0}, \rho_{\xi}\right) \subset R\left(U_{k}\right)$ (here it is used that $(\infty, \mathbf{0}) \notin U_{k}$ and hence $\left.\infty \notin V_{k}\right)$. By the compactness of $\operatorname{cl}\left(\tilde{V}_{k}\right)$, a finite number of $B\left(\xi, \rho_{\xi}\right), \xi \in \operatorname{cl}\left(\tilde{V}_{k}\right)$, is sufficient to cover $\operatorname{cl}\left(\tilde{V}_{k}\right)$. Taking the minimum of these $\rho_{\xi}$ implies that there exists $\rho^{(k)}>0$ such that $\tilde{V}_{k} \times D^{\prime}\left(\mathbf{0}, \rho^{(k)}\right) \subset R\left(U_{k}\right)$.

In a similar manner, we obtain $\rho_{0}^{(k)}, k=1, \ldots, N$ such that $\left(\tilde{V}_{k} \cap\right.$ $\left.\tilde{V}_{0}\right) \times D^{\prime}\left(\mathbf{0}, \rho_{0}^{(k)}\right) \subset R\left(U_{k} \cap U_{0}\right)$. Then let $\rho>0$ denote the minimum of the $2 N$ numbers $\rho^{(k)}, \rho_{0}^{(k)}, k=1, \ldots, N$. It has the property that for $k=1, \ldots, N$ we have $\tilde{V}_{k} \times D^{\prime}(\mathbf{0}, \rho) \subset R\left(U_{k}\right)$ and $\left(\tilde{V}_{0} \cap \tilde{V}_{k}\right) \times D^{\prime}(\mathbf{0}, \rho) \subset$ $R\left(U_{0} \cap U_{k}\right)$.

Therefore we can define bounded holomorphic functions $\tilde{D}_{j k}:=D_{j k}$ ○ $R^{-1}$ on $\left(\tilde{V}_{j} \cap \tilde{V}_{k}\right) \times D^{\prime}(\mathbf{0}, \rho)$ for $j, k \in\{0, \ldots, N\}$ not both equal to 0 . For completeness put $\tilde{D}_{00}=0$ on $\tilde{V}_{0} \times D^{\prime}(\mathbf{0}, \rho)$. Now we can apply Lemma 2.9 and obtain a family of bounded holomorphic functions $\tilde{F}_{j}: \tilde{V}_{j} \times D^{\prime}(\mathbf{0}, \rho) \rightarrow \mathbb{C}, j=0, \ldots, N$, such that $\tilde{D}_{j k}=\tilde{F}_{j}-\tilde{F}_{k}$ for $j, k=0, \ldots, N$. We have, moreover, for all $j \in\{0, \ldots, N\}$

$$
\left\|\tilde{F}_{j}\right\|_{\infty} \leq C \max \left\{\left\|\tilde{D}_{\ell k}\right\|_{\infty} \mid \ell, k \in\{0, \ldots, N\}\right\},
$$

where $C$ denotes the constant associated to the cover $\left\{\tilde{V}_{j}\right\}_{j=0, \ldots, N}$ in Lemma 2.8 . 
Now put $\tilde{U}_{j}=R^{-1}\left(\tilde{V}_{j} \times D^{\prime}(\mathbf{0}, \rho)\right)$ for $j \in\{1, \ldots, N\}, \tilde{U}_{0}=R^{-1}\left(\tilde{V}_{0} \times\right.$ $\left.D^{\prime}(\mathbf{0}, \rho)\right) \cap U_{0}$ and $F_{j}(p)=\tilde{F}_{j}(R(p))$ for $j \in\{0, \ldots, N\}, p \in \tilde{U}_{j}$. Then $\tilde{U}_{j} \cap E=\tilde{V}_{j} \times\{\mathbf{0}\}$ for $j=0, \ldots, N$ and hence $\tilde{U}_{j}, j=0, \ldots, N$, form an open cover of $E$. By construction, we have $\tilde{U}_{j} \subset U_{j}$ for $j=0, \ldots, N$ and $F_{j}(p)-F_{k}(p)=D_{j k}(p)$ for $p \in \tilde{U}_{j} \cap \tilde{U}_{j}$.

Remark 2.11. Lemma 2.10 can be extended to collections $\left(D_{i j}\right)_{i, j=0, \ldots, N}$ depending holomorphically upon parameters, that is $D_{i j}$ are holomorphic on $T \times U_{i j}, T$ an open subset of $\mathbb{C}^{k}$. We obtain collections $\left(F_{i}\right)_{i}$ of holomorphic functions on $T \times \tilde{U}_{i}$ and for $t \in T$ the estimates

$$
\max _{j \in\{0, \ldots, N\}} \sup _{p \in \tilde{U}_{j}}\left|F_{j}(t, p)\right| \leq C \max _{i, j \in\{0, \ldots, N\}} \sup _{p \in U_{i} \cap U_{j}}\left|D_{i j}(t, p)\right| \leq \infty .
$$

The proof remains essentially the same, one just has to apply Lemma 2.9 to $\tilde{V}_{j} \times D^{\prime}(\mathbf{0}, \rho) \times T$ instead of $\tilde{V}_{j} \times D^{\prime}(\mathbf{0}, \rho), j=0, \ldots, N$. Details are left to the reader.

\section{Classical and monomial asymptotics and summability}

Here we recall the notions and main properties of classical Poincaré and Gevrey asymptotics and summability in one variable (see, for instance, [Ram2, Sib1, Bal, Can]) and then the corresponding theory for monomial asymptotics of [CMS]. Our presentation follows essentially $[\mathbf{C M S}]$; the theory of monomial asymptotic expansions is presented for $d \geq 2$ variables instead of 2 and is rearranged and abbreviated.

3.1. Asymptotics in one variable. Let $E$ be a complex Banach space, with norm $\|\cdot\|_{E}$ and $\hat{f}(x)=\sum a_{n} x^{n} \in E \llbracket x \rrbracket$. A (open) sector in $\mathbb{C}$ is a set $V(a, b ; r)=\{x \in \mathbb{C}|a<\arg x<b, 0<| x \mid<r\}$. We will omit frequently $a, b, r$, and speak of a sector $V$. If $f: V \rightarrow E$ is holomorphic, $f$ is said to have $\hat{f}$ as an asymptotic expansion at the origin if for each $N \in \mathbb{N}$, there exists $C(N)>0$ such that

$$
\left\|f(x)-\sum_{n=0}^{N-1} a_{n} x^{n}\right\|_{E} \leq C(N)|x|^{N} \quad \text { in } V .
$$

The asymptotic expansion is $s$-Gevrey if, moreover, $C(N)$ can be chosen as $C(N)=K A^{N} N !^{s}$, with constants $K, A$. We will write $f \sim \hat{f}$ and $f \sim_{s} \hat{f}$ in the $s$-Gevrey case, respectively. Observe that $f \sim_{s} \hat{f}$ implies that the formal series $\hat{f}$ is $s$-Gevrey, i.e. there exist $C, A>0$ such that $\left|a_{n}\right| \leq C A^{n} n !^{s}$ for all $n \in \mathbb{N}$. The set of all such formal series will be denoted by $E \llbracket x \rrbracket_{s}$.

Asymptotic expansions are unique, and respect algebraic operations and differentiation. The so called Borel-Ritt-Gevrey theorem and Watson's lemma are of great importance. The following result collects them. 
Theorem 3.1. Let $V=V(a, b ; r), \hat{f} \in E \llbracket x \rrbracket_{s}$, and $s>0$. Then:

(1) If $b-a<s \pi$, there exists $f \in \mathcal{O}(V, E)$ such that $f \sim_{s} \hat{f}$.

(2) If $f \in \mathcal{O}(V, E)$ is such that $f \sim_{s} 0$, then there are positive constants such that

$$
\|f(x)\|_{E} \leq C \exp \left(-A /|x|^{1 / s}\right) .
$$

(3) If $b-a \geq s \pi$ and $f_{1}, f_{2} \in \mathcal{O}(V, E)$ have $\hat{f}$ as their $s$-Gevrey asymptotic expansion, then $f_{1}=f_{2}$.

Because of the above theorem, a function $f \in \mathcal{O}(V ; E)$ is uniquely determined by its $s$-Gevrey asymptotic expansion $\hat{f}$, provided that the opening of $V$ is larger than $s \pi$. If such a function exists for a formal series $\hat{f}$, then it is said to be $k$-summable in $V$ with $k=1 / s$ and $f$ is called the $k$-sum of $\hat{f}$ on $V$. More precisely:

Definition 3.2. Let $s>0, k=1 / s$, and $\hat{f} \in E \llbracket x \rrbracket_{s}$.

(1) The formal series $\hat{f}$ is called $k$-summable on $V=V(a, b ; r)$, if $b-a>$ $s \pi$ and there exists a function $f \in \mathcal{O}(V ; E)$ such that $f \sim_{s} \hat{f}$. The uniquely determined function $f$ is called the $k$-sum of $\hat{f}$ in the direction $\theta$.

(2) The formal series $\hat{f}$ is called $k$-summable in the direction $\theta \in \mathbb{R}$, if there exist $\delta, r>0$ such that $\hat{f}$ is $k$-summable on the sector $V\left(\theta-s \frac{\pi}{2}-\delta, \theta+s \frac{\pi}{2}+\delta ; r\right)$.

(3) The formal series $\hat{f}$ is simply called $k$-summable, if it is $k$-summable in every direction $\theta \in \mathbb{R}$ with finitely many exceptions $\bmod 2 \pi$.

The above notion of $k$-summability in a direction $\theta$ does not indicate how to obtain a sum from a given series; here the following characterization of $k$-summability helps.

Proposition 3.3. Given $\hat{f}(x)=\sum a_{n} x^{n} \in E \llbracket x \rrbracket_{s}$, it is k-summability in a direction $\theta$ if and only if the following statements hold.

(1) Its formal Borel transform $g(t)=\sum a_{n} t^{n} / \Gamma(1+n / k)$ is analytic in a neighborhood of the origin.

(2) The function $g$ can be continued analytically in some infinite sector $S=V(\theta-\delta, \theta+\delta ; \infty)$ containing the ray $\arg t=\theta$.

(3) It has exponential growth there, i.e. there are there are positive constants such that

$$
\|g(t)\|_{E} \leq C \exp \left(A /|t|^{k}\right)
$$

and hence the Laplace integral $f(x)=k x^{-k} \int_{\arg t=\tilde{\theta}} e^{-t^{k} / x^{k}} g(t) t^{k-1} d t$ defining the sum of $\hat{f}$ converges for $x$ in a certain sector $V=$ $V\left(\theta-\frac{\pi}{2 k}-\frac{\tilde{\delta}}{k}, \theta+\frac{\pi}{2 k}+\frac{\tilde{\delta}}{k} ; r\right), 0<\tilde{\delta}<\delta$, and suitably chosen $\tilde{\theta}$ close to $\theta$. It satisfies $f \sim_{s} \hat{f}$ on $V, s=1 / k$. 
We recall also the very useful characterization of functions having an $s$-Gevrey asymptotic expansion due to J.-P. Ramis and Y. Sibuya [Ram2, Sib2, Ram1].

Theorem 3.4. Suppose that the sectors $V_{j}=V\left(a_{j}, b_{j} ; r\right), 1 \leq j \leq m$, form a cover of the punctured disk $D(0 ; r)$. Given $f_{j}: V_{j} \rightarrow E$ bounded and analytic, assume that there is a constant $\gamma>0$ such that

$$
\left\|f_{j_{1}}(x)-f_{j_{2}}(x)\right\|_{E}=O\left(\exp \left(-\gamma /|x|^{1 / s}\right)\right)
$$

for $x \in V_{j_{1}} \cap V_{j_{2}}$, whenever this intersection is non-empty.

Then the functions $f_{j}$ have common $s$-Gevrey asymptotic expansions.

Conversely, if a function $f: V \rightarrow E$ having an $s$-Gevrey asymptotic expansion is given, then a cover $V_{j}, 1 \leq j \leq m$ and functions $f_{j}: V_{j} \rightarrow E$ can be found that satisfy estimates like (3.2) and $f=f_{1}$.

Such a family $f_{1}, \ldots, f_{m}$ is sometimes called a $k$-precise quasi-function.

In [Sib2] the following complement of the above theorem can be found:

Theorem 3.5. Suppose that the sectors $V_{j}=V\left(a_{j}, b_{j} ; r\right), 1 \leq j \leq m$, form a cover of the punctured disk $D(0 ; r)$. For couples $\left(j_{1}, j_{2}\right)$ with $V_{j_{1}} \cap V_{j_{2}} \neq \emptyset$, let holomorphic $d_{j_{1}, j_{2}}: V_{j_{1}} \cap V_{j_{2}} \rightarrow E$ be given that satisfy the cocycle condition $d_{j_{1}, j_{2}}+d_{j_{2}, j_{3}}=d_{j_{1}, j_{3}}$ whenever $V_{j_{1}} \cap V_{j_{2}} \cap V_{j_{3}} \neq \emptyset$ and estimates

$$
\left\|d_{j_{1}, j_{2}}(x)\right\|_{E}=O\left(\exp \left(-\gamma /|x|^{1 / s}\right)\right)
$$

for $j_{1}, j_{2} \in\{1, \ldots, m\}$ and $x \in V_{j_{1}} \cap V_{j_{2}}$ with some constants $s, \gamma>0$.

Then there exist bounded holomorphic functions $f_{j}: V_{j} \rightarrow E$ such that $d_{j_{1}, j_{2}}=f_{j_{1}}-f_{j_{2}}$ whenever $V_{j_{1}} \cap V_{j_{2}} \neq \emptyset$; moreover the functions $f_{j}$ have common s-Gevrey asymptotic expansions.

3.2. Monomial asymptotics. In [CMS $]$ the notion of monomial asymptotics in two variables was introduced in order to study doubly singular differential equations. We want to extend this notion to an arbitrary number of variables.

In the sequel, let $\boldsymbol{x}^{\boldsymbol{\alpha}}=x_{1}^{\alpha_{1}} \cdots x_{d}^{\alpha_{d}}$ denote a monomial in the $d$ variables $x_{1}, \ldots, x_{d}$. We begin by restating Corollary 2.5 in a slightly different way: $\mathbb{C}$ is replaced by an arbitrary $\mathbb{C}$-vector space $E$ and $P=\boldsymbol{x}^{\boldsymbol{\alpha}}$. Accordingly, we shall denote $\Delta\left(\boldsymbol{x}^{\boldsymbol{\alpha}}, E\right)=\left\{g=\sum g_{\boldsymbol{\beta}} x^{\boldsymbol{\beta}} \in E \llbracket \boldsymbol{x} \rrbracket ; g_{\boldsymbol{\beta}}=\right.$ 0 if $\left.\boldsymbol{\beta} \in \boldsymbol{\alpha}+\mathbb{N}^{d}\right\}$, as in (2.4).

Lemma 3.6. For any vector space E, there exists a canonical isomorphism

$$
T: E \llbracket \boldsymbol{x} \rrbracket \rightarrow \Delta\left(\boldsymbol{x}^{\boldsymbol{\alpha}}, E\right) \llbracket t \rrbracket
$$

with the property $(T f)\left(\boldsymbol{x}^{\boldsymbol{\alpha}}\right)=f$ for all series $f \in E \llbracket \boldsymbol{x} \rrbracket$. Here the symbol $(T f)\left(\boldsymbol{x}^{\boldsymbol{\alpha}}\right)$ means that $t$ is replaced by $\boldsymbol{x}^{\boldsymbol{\alpha}}$ in the series $T f$. 
In this statement, the name canonical emphasizes the independence of the linear form $\ell$. By abuse of notation, we use the same symbol $T$ for the analogous isomorphism $T: E\{\boldsymbol{x}\} \rightarrow \Delta\left(\boldsymbol{x}^{\boldsymbol{\alpha}}, E\right)\{t\}$ if $E$ is a normed vector space (and consequently, there is a notion of convergence).

For $r>0$ let $\mathcal{E}_{r}$ denote the Banach space of all functions $f \in \mathcal{O}_{b}(D(\mathbf{0} ; r))$ the series expansion of which $J(f) \in \Delta\left(\boldsymbol{x}^{\boldsymbol{\alpha}}\right)$. If $r^{\prime}<r$ there is a natural restriction map $\mathcal{E}_{r} \rightarrow \mathcal{E}_{r^{\prime}}$, linear and continuous. The image of $f \in \mathcal{E}_{r}$ will be denoted $\left.f\right|_{\mathcal{E}_{r^{\prime}}}$. Similarly, if $f(t)=\sum_{n=0}^{\infty} f_{n} t^{n} \in \mathcal{E}_{r} \llbracket t \rrbracket$ is a formal series, $\left.f(t)\right|_{\mathcal{E}_{r^{\prime}}}$ will represent $\left.\sum_{n=0}^{\infty} f_{n}\right|_{\mathcal{E}_{r^{\prime}}} t^{n}$.

In the subsequent lemma, we establish an analogue of the operator $T$ for functions defined on sectors in a monomial. This lemma generalizes the construction below Lemma 3.5 of $[\mathbf{C M S}]$ to an arbitrary number of variables.

We call "sector in $\boldsymbol{x}^{\boldsymbol{\alpha}}$ ", or $\boldsymbol{x}^{\boldsymbol{\alpha}}$-sector, a set $\Pi=\Pi(a, b ; \boldsymbol{R}) \subseteq(\mathbb{C} \backslash\{0\})^{d}$, $\left.\left.\boldsymbol{R}=\left(R_{1}, \ldots, R_{d}\right) \in\right] 0, \infty\right]^{d}$,

$$
\Pi=\left\{\boldsymbol{x} \in \mathbb{C}^{d}\left|a<\arg \left(\boldsymbol{x}^{\boldsymbol{\alpha}}\right)<b, 0<\right| x_{j} \mid<R_{j}, j=1, \ldots, d\right\} .
$$

Remark 3.7. Here and throughout this work, we will only consider sectors in $\mathbb{C}$, i.e. of opening not greater than $2 \pi$. So, in the definition of a $\boldsymbol{x}^{\boldsymbol{\alpha}}$-sector, and in subsequent definitions, we will assume implicitly that $b-a \leq 2 \pi$.

Lemma 3.8. Let $\Pi=\Pi(a, b ; \boldsymbol{R})$ a sector in $\boldsymbol{x}^{\boldsymbol{\alpha}}$ and $f: \Pi \rightarrow \mathbb{C} a$ holomorphic function. Then there is a uniquely determined holomorphic function $T f: V\left(a, b ; \boldsymbol{R}^{\boldsymbol{\alpha}}\right) \times D(\mathbf{0} ; \boldsymbol{R}) \rightarrow \mathbb{C}$ such that $J((T f)(t,).) \in$ $\Delta\left(\boldsymbol{x}^{\boldsymbol{\alpha}}\right)$ for any $t$ and $(T f)\left(\boldsymbol{x}^{\boldsymbol{\alpha}}, \boldsymbol{x}\right)=f(\boldsymbol{x})$. Moreover, if there is a function $\left.K:] 0, \boldsymbol{R}^{\boldsymbol{\alpha}}\right] \rightarrow \mathbb{R}_{+}$such that $|f(\boldsymbol{x})| \leq K\left(\left|\boldsymbol{x}^{\boldsymbol{\alpha}}\right|\right)$ for $\boldsymbol{x} \in \Pi$, then

$$
|(T f)(t, \boldsymbol{x})| \leq \frac{\boldsymbol{R}^{\alpha}}{|t|} K(|t|) \prod_{j=1}^{d}\left(1-\frac{\left|x_{j}\right|}{R_{j}}\right)^{-1} \text { for } t \in V\left(a, b ; \boldsymbol{R}^{\boldsymbol{\alpha}}\right), \boldsymbol{x} \in D(\mathbf{0} ; \boldsymbol{R}) .
$$

Remark 3.9. (1) Thus for $t \in V\left(a, b ; \boldsymbol{R}^{\boldsymbol{\alpha}}\right)$, the mapping $\boldsymbol{x} \mapsto(T f)(t, \boldsymbol{x})$ defines an element of any $\mathcal{E}_{r}, 0<r<\min _{j} R_{j}$. This element will be denoted by $\left.T f(t)\right|_{\mathcal{E}_{r}}$. Clearly $\left.T f\right|_{\mathcal{E}_{r}}: V\left(a, b ; \boldsymbol{R}^{\boldsymbol{\alpha}}\right) \rightarrow \mathcal{E}_{r}$ is holomorphic.

(2) The estimate could be improved by multiplying with $\left(1-\frac{\left|\boldsymbol{x}^{\boldsymbol{\alpha}}\right|}{\boldsymbol{R}^{\boldsymbol{\alpha}}}\right)$ on the right. We omit this factor, as it has no advantages in applications of the lemma.

Proof of Lemma 3.8: If $f: \Pi(a, b ; \boldsymbol{R}) \rightarrow \mathbb{C}$ is a holomorphic function and $\Pi\left(a^{\prime}, b^{\prime} ; \boldsymbol{R}^{\prime}\right)$ is some proper $\boldsymbol{x}^{\boldsymbol{\alpha}}$-subsector of $\Pi(a, b ; \boldsymbol{R})$, that is $a<a^{\prime}<$ $b^{\prime}<b$ and $0<R_{j}^{\prime}<R_{j}$ for $j=1, \ldots, d$, then we can establish a function $\left.K:] 0, \boldsymbol{R}^{\prime \boldsymbol{\alpha}}\right] \rightarrow \mathbb{R}_{+}$such that $|f(\boldsymbol{x})| \leq K\left(\left|\boldsymbol{x}^{\boldsymbol{\alpha}}\right|\right)$ for $\boldsymbol{x} \in \Pi\left(a^{\prime}, b^{\prime} ; \boldsymbol{R}^{\prime}\right)$. We 
can simply put

$$
K(s)=\max \{|f(\boldsymbol{x})| \mid \boldsymbol{x} \in \mathcal{M}(s)\},
$$

where $\mathcal{M}(s)=\left\{\boldsymbol{x} \in \operatorname{cl}\left(\Pi\left(a^{\prime}, b^{\prime} ; \boldsymbol{R}^{\prime}\right)\right)|| \boldsymbol{x}^{\boldsymbol{\alpha}} \mid=s\right\}$, because $\mathcal{M}(s)$ is a compact subset of $\Pi(a, b ; \boldsymbol{R})$. To see this observe that for $j=1, \ldots, d$ and $\boldsymbol{x} \in \mathcal{M}(s),\left|x_{j}\right|^{\alpha_{j}} \geq s \prod_{k \neq j} R_{k}^{\prime-\alpha_{k}}$ and hence none of the $x_{j}$ can be too close to 0 .

It is therefore sufficient to prove the lemma under the additional assumption that there exists a function $\left.K:] 0, \boldsymbol{R}^{\boldsymbol{\alpha}}\right] \rightarrow \mathbb{R}_{+}$such that $|f(\boldsymbol{x})| \leq K\left(\left|\boldsymbol{x}^{\boldsymbol{\alpha}}\right|\right)$ for $\boldsymbol{x} \in \Pi$. The uniqueness implies that we can define $T f$ for a holomorphic function on $\Pi(a, b ; \boldsymbol{R})$ by combining all the functions $T \tilde{f}$ obtained for the restrictions of $f$ to proper subsectors of $\Pi(a, b ; \boldsymbol{R})$.

The lemma had been proved in the case of a product of two variables in [CMS]. We give a proof for the general statement. Suppose first that $\boldsymbol{\alpha}=(1,1, \ldots, 1)$, i.e. the monomial is the product $\boldsymbol{x}^{\boldsymbol{\alpha}}=x_{1} \cdots x_{d}$. Then we show the statement with the improved estimate

$$
|(T f)(t, \boldsymbol{x})| \leq K(|t|) \prod_{j=1}^{d}\left(1-\frac{\left|x_{j}\right|}{R_{j}}\right)^{-1} \text { for } t \in V\left(a, b ; \boldsymbol{R}^{\boldsymbol{\alpha}}\right), \boldsymbol{x} \in D(\mathbf{0} ; \boldsymbol{R}) .
$$

Observe that we can assume without loss of generality that the radii coincide: $R_{j}=R$ for $j=1, \ldots, d$. Otherwise put $R=R_{1}$ and consider the function $\tilde{f}\left(x_{1}, \ldots, x_{d}\right)=f\left(x_{1}, x_{2} \frac{R_{2}}{R}, \ldots, x_{d} \frac{R_{d}}{R}\right)$ and $\tilde{K}(t)=$ $K\left(t R_{2} \cdots R_{d} / R^{d-1}\right)$; the radii are all reduced to $R$ now.

We now proceed similarly to $[\mathbf{C M S}]$, but have to treat Laurent series in several variables. Put $g\left(t, z_{2}, \ldots, z_{d}\right):=f\left(\frac{t}{z_{2} \cdots z_{d}}, z_{2}, \ldots, z_{d}\right)$. Then for fixed $t \in V:=V\left(a, b ; R^{d}\right), g\left(\boldsymbol{z}^{\prime} ; t\right)$ (with notation as in 2.1) is defined on the set of all $\boldsymbol{z}^{\prime}=\left(z_{2}, \ldots, z_{d}\right) \in \mathbb{C}^{d-1}$ such that $\left|\boldsymbol{z}^{\prime}\right|=$ $\max \left(\left|z_{2}\right|, \ldots,\left|z_{d}\right|\right)<R$ and $\left|z_{2} \cdots z_{d}\right|>\frac{|t|}{R}$. Applying several times the theorem on Laurent series expansions, we obtain that $g\left(\boldsymbol{z}^{\prime} ; t\right)=$ $\sum_{\boldsymbol{m} \in \mathbb{Z}^{d-1}} g_{\boldsymbol{m}}(t) \boldsymbol{z}^{\prime \boldsymbol{m}}$ with coefficients $g_{\boldsymbol{m}}(t)$ holomorphic on $V$ and that

$$
\left|g_{\boldsymbol{m}}(t)\right| \leq K(|t|) r_{2}^{-m_{2}} \cdots r_{d}^{-m_{d}}
$$

whenever $0<r_{2}, \ldots, r_{d}<R$ are such that their product $r_{2} \cdots r_{d}>\frac{|t|}{R}$. In order to get good estimates for these coefficients, we have to choose the $r_{j}$ in an optimal way.

In the case that one of the $m_{j}$ is negative, we choose $\ell$ such that the minimum of $m_{2}, \ldots, m_{d}$ is $m_{\ell}<0$ and rewrite (3.5) as

$$
\left|g_{\boldsymbol{m}}(t)\right| \leq K(|t|)\left(r_{2} \cdots r_{d}\right)^{-m_{\ell}} r_{2}^{m_{\ell}-m_{2}} \cdots r_{d}^{m_{\ell}-m_{d}} .
$$

As the differences $m_{\ell}-m_{j} \leq 0$ and one of them equals 0 in case $j=\ell$, we can choose $r_{j}=R$ if $j \neq \ell$ and $r_{\ell}$ arbitrary such that 
$r_{2} \cdots r_{d}>\frac{|t|}{R}$. Going over to the limit, we can as well assume that $r_{\ell}$ is chosen such that $r_{2} \cdots r_{d}=\frac{|t|}{R}$. Introducing the notation $\mu(\boldsymbol{m})=$ $m_{\ell}=\min \left(m_{2}, \ldots, m_{d}, 0\right)$ and $|\boldsymbol{m}|_{1}=m_{2}+\cdots+m_{d}$, we thus obtain in this case

$$
\left|g_{\boldsymbol{m}}(t)\right| \leq K(|t|)|t|^{-\mu(\boldsymbol{m})} R^{d \mu(\boldsymbol{m})-|\boldsymbol{m}|_{1}} .
$$

In the case where all $m_{j}$ are nonnegative, we choose $r_{j}=R$ for all $j$ and obtain $\left|g_{\boldsymbol{m}}(t)\right| \leq K(|t|) R^{-|\boldsymbol{m}|_{1}}$. So, (3.6) is valid for all $\boldsymbol{m} \in \mathbb{Z}^{d-1}$.

Now we put $h_{\boldsymbol{m}}(t):=t^{\mu(\boldsymbol{m})} g_{\boldsymbol{m}}(t)$ and obtain that $h_{\boldsymbol{m}}$ are holomorphic on $V$ and $\left|h_{\boldsymbol{m}}(t)\right| \leq K(|t|) R^{d \mu(\boldsymbol{m})-|\boldsymbol{m}|}$ for $t \in V$ and $\boldsymbol{m} \in \mathbb{Z}^{d-1}$. It is convenient to introduce $\phi: \mathbb{Z}^{d-1} \rightarrow \mathbb{N}^{d}$ by $\phi(\boldsymbol{m})=\left(-\mu(\boldsymbol{m}), m_{2}-\right.$ $\left.\mu(\boldsymbol{m}), \ldots, m_{d}-\mu(\boldsymbol{m})\right)$. Observe that $\phi$ is a bijection between $\mathbb{Z}^{d-1}$ and the set $\mathcal{M}_{d}$ of all $\boldsymbol{n}=\left(n_{1}, \ldots, n_{d}\right) \in \mathbb{N}^{d}$ such that at least one of the $n_{j}$ vanishes; moreover $|\phi(\boldsymbol{m})|_{1}=|\boldsymbol{m}|_{1}-d \mu(\boldsymbol{m})$. Now we define for $t \in V$

$$
(T f)(t, \boldsymbol{x})=\sum_{\boldsymbol{m} \in \mathbb{Z}^{d-1}} h_{\boldsymbol{m}}(t) \boldsymbol{x}^{\phi(\boldsymbol{m})} .
$$

As all $\phi(\boldsymbol{m})$ are in $\mathcal{M}_{d}$, we obtain $J((T f)(t,).) \in \Delta\left(\boldsymbol{x}^{\boldsymbol{\alpha}}\right)$. Next, we have to show the convergence of the series if $\left|x_{j}\right|<R$ for all $j$. Using $\phi\left(\mathbb{Z}^{d-1}\right)=\mathcal{M}_{d}$, we estimate

$$
\sum_{\boldsymbol{m} \in \mathbb{Z}^{d-1}}\left|h_{\boldsymbol{m}}(t)\right|\left|\boldsymbol{x}^{\phi(\boldsymbol{m})}\right| \leq K(|t|) \sum_{\boldsymbol{n} \in \mathcal{M}_{d}}\left(\frac{|\boldsymbol{x}|}{R}\right)^{|\boldsymbol{n}|_{1}} \leq K(|t|) \prod_{j=1}^{d}\left(1-\frac{\left|x_{j}\right|}{R}\right)^{-1}
$$

and thus the convergence of the series and the estimate of the theorem. This also implies that $T f$ is analytic for $t \in V, \boldsymbol{x} \in D(\mathbf{0} ; R)$. The fact that $(T f)\left(\boldsymbol{x}^{\boldsymbol{\alpha}}, \boldsymbol{x}\right)=f(\boldsymbol{x})$ follows easily from the construction

$$
\begin{aligned}
\sum_{\boldsymbol{m} \in \mathbb{Z}^{d-1}} h_{\boldsymbol{m}}\left(\boldsymbol{x}^{\boldsymbol{\alpha}}\right) \boldsymbol{x}^{\phi(\boldsymbol{m})} & =\sum_{\boldsymbol{m} \in \mathbb{Z}^{d-1}} g_{\boldsymbol{m}}\left(\boldsymbol{x}^{\boldsymbol{\alpha}}\right) \boldsymbol{x}^{\mu(\boldsymbol{m}) \boldsymbol{\alpha}+\phi(\boldsymbol{m})} \\
& =\sum_{\boldsymbol{m} \in \mathbb{Z}^{d-1}} g_{\boldsymbol{m}}\left(\boldsymbol{x}^{\boldsymbol{\alpha}}\right) \boldsymbol{x}^{\prime \boldsymbol{m}}=f(\boldsymbol{x}) .
\end{aligned}
$$

We now reduce the general case to the one treated above. Suppose that $\alpha_{1}>1$ and let $\xi=e^{2 \pi i / \alpha_{1}}$. Observe that $\boldsymbol{x} \in \Pi$ implies $\boldsymbol{x}^{(k)}:=\left(\xi^{k} x_{1}, \boldsymbol{x}^{\prime}\right) \in \Pi$ for $k=0, \ldots, \alpha_{1}-1$ and therefore there are uniquely determined functions $F_{0}, \ldots, F_{\alpha_{1}-1}$ defined on the sector $a<\arg \left(z x_{2}^{\alpha_{2}} \cdots x_{d}^{\alpha_{d}}\right)<b, 0<|z|<R_{1}^{\alpha_{1}}, 0<\left|x_{j}\right|<R_{j}$, for $j=2, \ldots, d$ in the monomial $z x_{2}^{\alpha_{2}} \cdots x_{d}^{\alpha_{d}}$ such that

$$
f(\boldsymbol{x})=\sum_{j=0}^{\alpha_{1}-1} x_{1}^{j} F_{j}\left(x_{1}^{\alpha_{1}}, \boldsymbol{x}^{\prime}\right)
$$


The functions $F_{j}$ can be determined by the Vandermonde system

$$
f\left(\boldsymbol{x}^{(k)}\right)=\sum_{j=0}^{\alpha_{1}-1} \xi^{j k} x_{1}^{j} F_{j}\left(x_{1}^{\alpha_{1}}, \boldsymbol{x}^{\prime}\right), \quad k=0, \ldots, \alpha_{1}-1 .
$$

Hence,

$$
x_{1}^{j} F_{j}\left(x_{1}^{\alpha_{1}}, \boldsymbol{x}^{\prime}\right)=\frac{1}{\alpha_{1}} \sum_{k=0}^{\alpha_{1}-1} \xi^{-j k} f\left(\boldsymbol{x}^{(k)}\right), \quad j=0, \ldots, \alpha_{1}-1,
$$

and therefore $\left|x_{1}^{\alpha_{1}} F_{j}\left(x_{1}^{\alpha_{1}}, x_{2}, \ldots, x_{d}\right)\right| \leq R_{1}^{\alpha_{1}-j} K\left(\left|\boldsymbol{x}^{\boldsymbol{\alpha}}\right|\right)$ for $j=0, \ldots, \alpha_{1}-$ $1, \boldsymbol{x} \in \Pi$. Continuing in this way we prove that

$$
f(x)=\sum_{\mathbf{0} \leq \boldsymbol{\beta}<\boldsymbol{\alpha}} \boldsymbol{x}^{\boldsymbol{\beta}} F_{\boldsymbol{\beta}}\left(x_{1}^{\alpha_{1}}, \ldots, x_{d}^{\alpha_{d}}\right),
$$

where summation is over all integer vectors $\boldsymbol{\beta} \in \mathbb{Z}^{d}, 0 \leq \beta_{j}<\alpha_{j}$ for all $j$, and where the functions $F_{\boldsymbol{\beta}}$ satisfy

$$
\left|\boldsymbol{x}^{\alpha} F_{\boldsymbol{\beta}}\left(x_{1}^{\alpha_{1}}, \ldots, x_{d}^{\alpha_{d}}\right)\right| \leq \boldsymbol{R}^{\boldsymbol{\alpha}-\boldsymbol{\beta}} K\left(\left|\boldsymbol{x}^{\boldsymbol{\alpha}}\right|\right) .
$$

Now the situation is reduced to functions $F_{\boldsymbol{\beta}}$, satisfying

$$
\left|F_{\boldsymbol{\beta}}\left(u_{1}, \ldots, u_{d}\right)\right| \leq \boldsymbol{R}^{\boldsymbol{\alpha}-\boldsymbol{\beta}} \frac{K\left(\left|u_{1} \cdots u_{d}\right|\right)}{\left|u_{1} \cdots u_{d}\right|}
$$

on a $\left(u_{1} \cdots u_{d}\right)$-sector $\tilde{\Pi}$. Using the first part of the proof, especially (3.4) for each $F_{\boldsymbol{\beta}}$, and then combining them using (3.8) implies the statement. We just have to use the formula

$$
\sum_{\mathbf{0} \leq \boldsymbol{\beta}<\boldsymbol{\alpha}} \boldsymbol{x}^{\boldsymbol{\beta}} \boldsymbol{R}^{-\boldsymbol{\beta}} \prod_{j=1}^{d}\left(1-\frac{\left|x_{j}^{\alpha_{j}}\right|}{R_{j}^{\alpha_{j}}}\right)^{-1}=\prod_{j=1}^{d}\left(1-\frac{\left|x_{j}\right|}{R_{j}}\right)^{-1} .
$$

The proof of the uniqueness can be given following the same steps as in the construction of $T f$. Details are left to the reader. An alternative proof is given, in the context of asymptotic expansions with respect to a germ, at the end of the proof of Theorem 4.7, at the end of Section 5.

Example 3.10. The following example due to S. Kamimoto shows that if one of the $\alpha_{i}>1$, then the estimate for $T f$ cannot be as good as in (3.4) in the case of a "simple" product $x_{1} \cdots x_{d}$.

Consider the monomial $\boldsymbol{x}^{\boldsymbol{\alpha}}=x_{1}^{2} x_{2}$ and a small $\boldsymbol{x}^{\boldsymbol{\alpha}}$-sector $\Pi=$ $\Pi(-\delta, \delta ; R), \delta, R>0$. Define $f: \Pi \rightarrow \mathbb{C}$ by the principal value $x_{2}^{1 / 2}$ if $\arg x_{1}$ and $\arg x_{2}$ are both small and extend this function to all of $\Pi$ by analytical continuation. This is possible as for any path $\gamma:[0,1] \rightarrow \Pi$, $\gamma(s)=\left(\gamma_{1}(s), \gamma_{2}(s)\right)$, we must have $\left|2 \arg \gamma_{1}(s)+\arg \gamma_{2}(s)\right|<\delta$. Hence if we start with $\arg \gamma_{j}(s) \approx 0$ and $\gamma_{2}$ has made one tour of $x_{2}=0$ and thus reached $\arg \gamma_{2}(s) \approx 2 \pi$, then we have $\arg \gamma_{1}(s) \approx-\pi$ and are far 
away from the starting point of the path. After two tours of $\gamma_{2}$ around $x_{2}=0$, we have $\arg \gamma_{1}(s) \approx 2 \pi$ and $\arg \gamma_{2}(s) \approx 4 \pi$ and are again (with respect to the arguments) close to the starting points of the path. The values of $f$ obtained by analytic continuation of $x_{2}^{1 / 2}$ are also close to the original ones as $\arg x_{2}$ has been changed by about $4 \pi$.

Thus we have an analytic function $f: \Pi \rightarrow \mathbb{C}$ that is bounded and satisfies $f\left(-x_{1}, x_{2}\right)=-f\left(x_{1}, x_{2}\right)$ for $\left(x_{1}, x_{2}\right) \in \Pi$. The unique function $T f$ of Lemma 3.8 is apparently $\operatorname{Tf}\left(t,\left(x_{1}, x_{2}\right)\right)=t^{-1 / 2} x_{1} x_{2}$ (with the principal value of $t^{-1 / 2}$ ) and this function is not bounded as $t \rightarrow 0$.

In the above example, the $P$-sector is connected. A simpler example where $\Pi$ has several connected components is given in the one variable case by the monomial $x^{2}$. Consider the $x^{2}$-sector $\Pi(-\delta, \delta, R)$ which has the two components $|\arg x|<\delta / 2$ respectively $|\arg x-\pi|<\delta / 2$. A bounded holomorphic function can be defined by having the value 1 on one component and the value -1 on the other. The corresponding function $T f$ is apparently $T f(t, x)=t^{-1 / 2} x$ and also unbounded as $t \rightarrow 0$.

Now we are in a position to define monomial asymptotics.

Definition/Proposition 3.11. Let $f$ be a bounded holomorphic function on $\Pi=\Pi(a, b ; R)$ and $\hat{f} \in \hat{\mathcal{O}}$. We will say that $f$ has $\hat{f}$ as asymptotic expansion at the origin in $\boldsymbol{x}^{\boldsymbol{\alpha}}$ if there exists $0<\tilde{R} \leq R$ such that $T \hat{f}(t)=\sum_{n=0}^{\infty} g_{n} t^{n} \in \mathcal{E}_{\tilde{R}} \llbracket t \rrbracket$ and one of the following equivalent conditions is satisfied:

(1) For every $r \in] 0, \tilde{R}\left[\right.$ one has $\left.\left.T f(t)\right|_{\mathcal{E}_{r}} \sim T \hat{f}(t)\right|_{\mathcal{E}_{r}}$ as $V\left(a, b ; r^{n}\right) \ni$ $t \rightarrow 0$ in the sense of (3.1).

(2) For every $0<r<\tilde{R}$ and every $N$, there exists $C(N, r)$ such that for all $\boldsymbol{x} \in \Pi(a, b ; r)$

$$
\left|f(\boldsymbol{x})-\sum_{n=0}^{N-1} g_{n}(\boldsymbol{x}) \boldsymbol{x}^{n \boldsymbol{\alpha}}\right| \leq C(N, r)\left|\boldsymbol{x}^{N \boldsymbol{\alpha}}\right| .
$$

Analogously, we define the notion of $s$-Gevrey asymptotic expansion if $T \hat{f}$ is an $s$-Gevrey formal series (with coefficients in $\mathcal{E}_{\tilde{R}}$ ) and $T f \sim_{s} T \hat{f}$ or, equivalently, $C(N, r)$ can be chosen as $L(r) A(r)^{N} N !^{s}$.

Proof: It suffices to prove that the second condition implies the first, the converse is trivial. For that purpose, consider the function $\delta(\boldsymbol{x})=$ $f(\boldsymbol{x})-\sum_{n=0}^{N} g_{n}(\boldsymbol{x}) \boldsymbol{x}^{n \boldsymbol{\alpha}}$. We can apply Lemma 3.8 with $K(u)=u^{N+1}$ and obtain for $0<r^{\prime}<r<\tilde{R}$

$$
\left|(T f)(t, \boldsymbol{x})-\sum_{n=0}^{N} g_{n}(\boldsymbol{x}) t^{n}\right| \leq C(N+1, r)|t|^{N}\left(1-\frac{r^{\prime}}{r}\right)^{-d} .
$$


So, we have

$$
\begin{aligned}
\left|(T f)(t, \boldsymbol{x})-\sum_{n=0}^{N-1} g_{n}(\boldsymbol{x}) t^{n}\right| & \leq\left|(T f)(t, \boldsymbol{x})-\sum_{n=0}^{N} g_{n}(\boldsymbol{x}) t^{n}\right|+\left|g_{N}(\boldsymbol{x}) t^{N}\right| \\
& \leq C(N+1, r)|t|^{N}\left(1-\frac{r^{\prime}}{r}\right)^{-d}+\left\|g_{N}(\boldsymbol{x})\right\||t|^{N} .
\end{aligned}
$$

Remark 3.12. Let us note that, in the Gevrey case, the series $T \hat{f}(t)$ automatically turns out to be $s$-Gevrey. In fact, from the inequalities

$$
\left|(T f)(t, \boldsymbol{x})-\sum_{n=0}^{N} g_{n}(\boldsymbol{x}) t^{n}\right| \leq L(r) A(r)^{N+1}(N+1) !^{s}|t|^{N}\left(1-\frac{r^{\prime}}{r}\right)^{-d}
$$

we obtain that

$$
\begin{aligned}
\left|g_{N}(\boldsymbol{x})\right| & \leq L(r) A(r)^{N+1}(N+1) !^{s}\left(1-\frac{r^{\prime}}{r}\right)^{-d}+L(r) A(r)^{N} N !^{s}\left(1-\frac{r^{\prime}}{r}\right)^{-d} \frac{1}{|t|} \\
& =L(r)\left(1-\frac{r^{\prime}}{r}\right)^{-d} A(r)^{N} N !^{s}\left[A(r)(N+1)^{s}+\frac{1}{|t|}\right] .
\end{aligned}
$$

Fixing $t$ with $|t|=\frac{r}{2}$ yields Gevrey bounds for $g_{N}(\boldsymbol{x})$.

In the rest of this subsection, we recall the properties of Gevrey asymptotic expansions in a monomial from [CMS], but state and prove them in the general setting - whereas [CMS] only consider the monomial $x_{1} x_{2}$. Since we have the main Lemma 3.8 in the general setting, the generalization is straightforward.

As in the single variable case, functions Gevrey asymptotic to 0 in a monomial are exponentially small.

Lemma 3.13. If $f \in \mathcal{O}(\Pi ; E)$ has an $s$-Gevrey asymptotic expansion in $\boldsymbol{x}^{\boldsymbol{\alpha}}$ where $\hat{f}=0$, then, for all sufficiently small $R^{\prime}>0$ there exist $C, B>0$ such that on $\tilde{\Pi}$

$$
|f(\boldsymbol{x})| \leq C \exp \left(-\frac{B}{\left|\boldsymbol{x}^{\boldsymbol{\alpha}}\right|^{1 / s}}\right) \quad \text { for } \boldsymbol{x} \in \Pi,|\boldsymbol{x}|<R^{\prime} .
$$

Proof: As in the classical case, we choose $N$ close to the optimal value $\left(A\left|\boldsymbol{x}^{\boldsymbol{\alpha}}\right|\right)^{-1 / s}$ in the definition of an $s$-Gevrey asymptotic expansion in $\boldsymbol{x}^{\boldsymbol{\alpha}}$. Stirling's formula yields the statement.

Using the first condition in the definition of an $s$-Gevrey asymptotic expansion in $\boldsymbol{x}^{\boldsymbol{\alpha}}$ and using Lemma 3.8 with $K(u)=\exp \left(-\gamma / u^{1 / s}\right)$, the theorem of Ramis-Sibuya (Theorem 3.4) (together with Theorem 3.1 (1)) immediately implies: 
Theorem 3.14. Suppose that the sectors $\Pi_{j}=\Pi\left(a_{j}, b_{j} ; r\right), 1 \leq j \leq m$ in $\boldsymbol{x}^{\boldsymbol{\alpha}}$, form a cover of $D(\mathbf{0} ; r) \backslash\left\{\boldsymbol{x} ; \boldsymbol{x}^{\boldsymbol{\alpha}}=\mathbf{0}\right\}$. Given $f_{j}: \Pi_{j} \rightarrow E$ bounded and analytic, assume that for every subsector $\Pi^{\prime}$ of $\Pi_{j_{1}} \cap \Pi_{j_{2}}$ (provided that $\Pi_{j_{1}} \cap \Pi_{j_{2}} \neq \emptyset$ ) there is a constant $\gamma\left(\Pi^{\prime}\right)>0$ such that

$$
\left|f_{j_{1}}(\boldsymbol{x})-f_{j_{2}}(\boldsymbol{x})\right|=O\left(\exp \left(-\gamma\left(\Pi^{\prime}\right) /\left|\boldsymbol{x}^{\boldsymbol{\alpha}}\right|^{1 / s}\right)\right)
$$

for $\boldsymbol{x} \in \Pi^{\prime}$. Then the functions $f_{j}$ have asymptotic expansions in $\boldsymbol{x}^{\boldsymbol{\alpha}}$ with a common right hand side and the expansions are $s$-Gevrey.

Conversely, if a function $f: \Pi \rightarrow E$ having an $s$-Gevrey asymptotic expansion in $\boldsymbol{x}^{\boldsymbol{\alpha}}$ is given, then a cover $\Pi_{j}, 1 \leq j \leq m$ and functions $f_{j}: \Pi_{j} \rightarrow E$ can be found that satisfy estimates like (3.9) and $f=f_{1}$.

As a consequence, Gevrey asymptotics in a monomial are compatible with the elementary operations (sum, product,...). This is not obvious from the definition, except for addition.

Also, a Watson's lemma for Gevrey asymptotics in a monomial follows from Lemma 3.8 and the one-variable version in Theorem 3.1 (2).

Theorem 3.15. Let $\Pi=\Pi(a, b ; R)$ be a sector in $\boldsymbol{x}^{\boldsymbol{\alpha}}$ with $b-a>s \pi$ and suppose that $f \in \mathcal{O}(\Pi ; E)$ has $\hat{f}=0$ as its $s$-Gevrey asymptotic expansion. Then $f \equiv 0$.

Definition 3.16. Let $s>0, k=1 / s$ and a formal series $\hat{f}(\boldsymbol{x})=$ $\sum_{\boldsymbol{m} \in \mathbb{N}^{d}} a_{\boldsymbol{m}} x^{\boldsymbol{m}}$ be given.

(1) We say that $\hat{f}$ is $k$-summable in $\boldsymbol{x}^{\boldsymbol{\alpha}}$ on $\Pi=\Pi(a, b ; R)$ if $b-a>s \pi$ and there exists a holomorphic bounded function $f: \Pi \rightarrow E$ such that $f$ has $\hat{f}$ as its $s$-Gevrey asymptotic expansion in $\boldsymbol{x}^{\boldsymbol{\alpha}}$ on $\Pi$ in the sense of Definition/Proposition 3.11. Then $f$ is called the $k$-sum of $\hat{f}$ in $\boldsymbol{x}^{\boldsymbol{\alpha}}$ on $\Pi$. If it exists, it is unique, by Theorem 3.15.

(2) The formal series $\hat{f}$ is called $k$-summable in $\boldsymbol{x}^{\boldsymbol{\alpha}}$ in the direction $\theta \in$ $\mathbb{R}$, if there exist $\delta, r>0$ such that $\hat{f}$ is $k$-summable in $\boldsymbol{x}^{\boldsymbol{\alpha}}$ on the sector $\Pi\left(\theta-s \frac{\pi}{2}-\delta, \theta+s \frac{\pi}{2}+\delta ; r\right)$ in $\boldsymbol{x}^{\boldsymbol{\alpha}}$.

(3) The formal series $\hat{f}$ is simply called $k$-summable, if it is $k$-summable in every direction $\theta \in \mathbb{R}$ with finitely many exceptions mod $2 \pi$ (called singular directions).

The first condition in Definition/Proposition 3.11 shows that $\hat{f}$ is $k$-summable in $\boldsymbol{x}^{\boldsymbol{\alpha}}$ on $\Pi(a, b ; R)$ if and only if the formal series $T \hat{f}=$ $\sum_{n=0}^{\infty} g_{n} t^{n}$ has coefficients in $\mathcal{E}_{r}$ and if it is $k$-summable on $V\left(a, b ; r^{d}\right)$ for $r>0$ sufficiently small as series in one variable with coefficients in a Banach space. This allows us to carry over classical theorems to $k$-summability in a monomial. 
It would be tempting to define summability in a monomial (and also Gevrey asymptotics in a monomial) using only a fixed radius $r>0$, but an example in [CMS] shows that $r$ might have to be chosen smaller and smaller if the direction $\theta$ approaches a singular direction.

\section{Asymptotics with respect to an analytic germ}

Consider a germ of analytic function $P(\boldsymbol{x}) \in \mathcal{O}=\mathbb{C}\left\{x_{1}, \ldots, x_{d}\right\}$, not a unit (i.e. $P(\mathbf{0})=0$ ) and not identically vanishing, defined in some neighbourhood of $\mathbf{0} \in \mathbb{C}^{d}$, say in $D(\mathbf{0} ; \rho)$.

Definition 4.1. A sequence $\left\{f_{n}\right\}_{n=0}^{\infty}$ in $\mathcal{O}_{b}(D(0 ; \rho))$ is an asymptotic sequence (for $\hat{f})$ if $J\left(f_{n}\right)$ converges in the $\mathfrak{m}$-adic topology of $\hat{\mathcal{O}}=\mathbb{C} \llbracket \boldsymbol{x} \rrbracket$ towards an element $\hat{f} \in \hat{\mathcal{O}}$.

If, moreover, $J\left(f_{n}\right) \equiv \hat{f} \bmod P^{n} \hat{\mathcal{O}}$ for all $n$, then we will say that $\left\{f_{n}\right\}_{n}$ is a $P$-asymptotic sequence (for $\left.\hat{f}\right)$.

If $\hat{f} \in \hat{\mathcal{O}}$ is the limit of some $P$-asymptotic sequence, then we say that $\hat{f}$ is a P-asymptotic series.

Definition 4.2. Given $a<b, 0<R_{j} \leq+\infty, j=1, \ldots, d, \boldsymbol{R}=$ $\left(R_{1}, \ldots, R_{d}\right)$, the $P$-sector $\Pi_{P}(a, b ; \boldsymbol{R})$ is the set

$$
\Pi_{P}(a, b ; \boldsymbol{R})=\left\{\boldsymbol{x} \in \mathbb{C}^{d} ; a<\arg P(\boldsymbol{x})<b, 0<\left|x_{j}\right|<R_{j} \text { for } j=1, \ldots, d\right\} .
$$

By abuse of notation, we sometimes write $\Pi_{P}(a, b ; R)$ for $\Pi_{P}(a, b$; $(R, R, \ldots, R))$.

Definition 4.3. Given a $P$-sector $\Pi, f \in \mathcal{O}(\Pi)$, and $\hat{f} \in \mathbb{C} \llbracket \boldsymbol{x} \rrbracket$, we will say that $\hat{f}$ is the $P$-asymptotic expansion of $f$ on $\Pi$ if there exist $\rho>0$ and a $P$-asymptotic sequence $\left\{f_{n}\right\}_{n=1}^{\infty}$ in $\mathcal{O}_{b}(D(\mathbf{0} ; \rho))$ for $\hat{f}$, such that, for every $n \in \mathbb{N}$, there exists $K_{n}>0$ such that

$$
\left|f(\boldsymbol{x})-f_{n}(\boldsymbol{x})\right| \leq K_{n}|P(\boldsymbol{x})|^{n}
$$

on $D(\mathbf{0} ; \rho) \cap \Pi$. We will denote this by $f \sim_{\Pi}^{P} \hat{f}$. Observe that $\hat{f}$ is a $P$-asymptotic series in this case.

Remark 4.4. (1) In Theorem 4.9, we will show that the above definition is equivalent to statements that reduce to Definition/Proposition 3.11 in the case of a monomial.

(2) If $U$ is a unit and $Q=U P$ then it is immediate to verify that $f$ has a series $\hat{f}$ as a $P$-asymptotic expansion if and only if $f$ has the same series as $Q$-asymptotic expansion.

(3) Specialization. Consider a disk $D\left(\mathbf{0} ; \rho^{\prime}\right) \in \mathbb{C}^{m}$, and $Q: D\left(\mathbf{0} ; \rho^{\prime}\right) \rightarrow \mathbb{C}^{d}$ such that $Q(\mathbf{0}) \in D(\mathbf{0} ; \rho) \subseteq \mathbb{C}^{d}$, and $P \circ Q(\mathbf{0})=0$ but $P \circ Q \not \equiv 0$. Let 
$f \in \mathcal{O}\left(\Pi_{P}(a, b ; R)\right)$ and consider a $P$-asymptotic sequence $\left\{f_{n}\right\}_{n=1}^{\infty}$ for $f$. There exists $R^{\prime}>0$ such that, if $\mathbf{y} \in \mathbb{C}^{m}$ verifies $0<\left|y_{i}\right|<R^{\prime}$ for every $i, 1 \leq i \leq m$, then $Q(\mathbf{y}) \in D(\mathbf{0} ; R) \subseteq \mathbb{C}^{d}$. Under these conditions, $f \circ Q$ is well defined on a $P \circ Q$-sector $\tilde{\Pi}=\Pi_{P \circ Q}\left(a, b ; R^{\prime}\right)$ and has the sequence $\left\{f_{n} \circ Q\right\}_{n}$ as $P \circ Q$-asymptotic sequence.

This applies in particular when $Q(\mathbf{0})=\mathbf{0}$. Another interesting special case of this property can be given in the context of monomial asymptotic expansions, i.e. $P=\boldsymbol{x}^{\boldsymbol{\alpha}}$, and $Q: \mathbb{C} \rightarrow \mathbb{C}^{d}$ is defined by $Q(x)=\left(x, t_{2}, \ldots, t_{d}\right)$, with $\left(t_{2}, \ldots, t_{d}\right) \in \mathbb{C}^{d-1}, 0<\left|t_{i}\right|<R$. We obtain that monomial asymptotic expansions can be specialized, fixing the values of some of the variables.

(4) The notion of $P$-asymptotic expansion agrees with the usual notion of asymptotic expansion in one variable if $P=x$. Indeed, suppose that $f$ is a holomorphic function defined on a sector $V$, and that there is a family of holomorphic functions $\left\{f_{n}\right\}_{n}$, defined on a common neighbourhood of the origin $D\left(\mathbf{0}^{\prime} ; \rho\right)$, and such that there exists $C_{n}$ with

$$
\left|f(x)-f_{n}(x)\right| \leq C_{n}|x|^{n}
$$

on $V \cap D(\mathbf{0} ; \rho)$. The sequence $\left\{f_{n}\right\}_{n}$ turns out to be an asymptotic sequence. Indeed, observe that

$$
\left|f_{n}(x)-f_{n+1}(x)\right| \leq\left|f_{n}(x)-f(x)\right|+\left|f(x)-f_{n+1}(x)\right| \leq\left(C_{n}+C_{n+1}|x|\right)|x|^{n},
$$

and therefore the meromorphic functions $\left(f_{n}(x)-f_{n+1}(x)\right) / x^{n}$ are bounded on $V \cap D(\mathbf{0} ; \rho)$, thus holomorphic at the origin. Therefore we have $J_{n-1}\left(f_{n}\right)=J_{n-1}\left(f_{n+1}\right)$ for all $n$ and $J\left(f_{n}\right)$ converges in the m-adic topology of $\mathbb{C} \llbracket x \rrbracket$ towards some series $\hat{f}$, such that $J_{n-1}\left(f_{m}\right)=J_{n-1}(\hat{f})$ whenever $m \geq n$.

As we have $\left|f_{n}(x)-J_{n-1}\left(f_{n}\right)(x)\right| \leq K_{n}|x|^{n}$ for every $n \in \mathbb{N}$ with some $K_{n}$, we finally obtain

$\left|f(x)-J_{n-1}(\hat{f})(x)\right| \leq\left|f(x)-f_{n}(x)\right|+\left|f_{n}(x)-J_{n-1}\left(f_{n}\right)(x)\right| \leq\left(C_{n}+K_{n}\right)|x|^{n}$, on $V \cap D\left(\mathbf{0} ; \rho^{\prime}\right)$.

The converse is trivial.

Lemma 4.5. (1) If a sequence $\left\{f_{n}\right\}_{n}$ of functions on some polydisk $D(\mathbf{0} ; \rho)$ and a function $f$ on some P-sector satisfy the inequalities (4.1), then $\left\{f_{n}\right\}_{n}$ is a P-asymptotic sequence.

(2) The P-asymptotic expansion of a function $f$ on a P-sector, if it exists, is unique.

Proof: For (1): Such a sequence satisfies for all $n \in \mathbb{N}$

$$
\begin{aligned}
\left|f_{n}(\boldsymbol{x})-f_{n+1}(\boldsymbol{x})\right| & \leq\left|f_{n}(\boldsymbol{x})-f(\boldsymbol{x})\right|+\left|f(\boldsymbol{x})-f_{n+1}(\boldsymbol{x})\right| \\
& \leq\left(K_{n}+K_{n+1}|P(\boldsymbol{x})|\right)|P(\boldsymbol{x})|^{n} \leq K_{n}^{\prime}|P(\boldsymbol{x})|^{n}
\end{aligned}
$$


on the $P$-sector $\Pi$ mentioned in the statement. By Lemma 2.2, $P(\boldsymbol{x})^{n}$ divides $f_{n}(\boldsymbol{x})-f_{n+1}(\boldsymbol{x})$ for all $n$. As $P(\mathbf{0})=0,\left\{f_{n}\right\}_{n \in \mathbb{N}}$ is a Cauchy sequence for the $\mathfrak{m}$-adic topology and converges to some $\hat{f} \in \hat{\mathcal{O}}$. Moreover, $f_{n} \equiv \hat{f} \bmod P^{n} \hat{\mathcal{O}}$ for all $n$ and the statement follows.

For (2): Let $\left\{f_{n}\right\}_{n},\left\{\tilde{f}_{n}\right\}_{n}$ be two asymptotic sequences on a $P$-sector $\Pi$, such that a family of constants $C_{n}>0$ exists satisfying

$$
\begin{aligned}
\left|f(\boldsymbol{x})-f_{n}(\boldsymbol{x})\right| & \leq C_{n}|P(\boldsymbol{x})|^{n}, \\
\left|f(\boldsymbol{x})-\tilde{f}_{n}(\boldsymbol{x})\right| & \leq C_{n}|P(\boldsymbol{x})|^{n} .
\end{aligned}
$$

Then,

$$
\left|f_{n}(\boldsymbol{x})-\tilde{f}_{n}(\boldsymbol{x})\right| \leq 2 C_{n}|P(\boldsymbol{x})|^{n}
$$

on $\Pi$, and by Lemma $2.2, P(\boldsymbol{x})^{n}$ divides $f_{n}(\boldsymbol{x})-\tilde{f}_{n}(\boldsymbol{x})$. So, the families $\left\{f_{n}\right\}_{n},\left\{\tilde{f}_{n}\right\}$ have the same limit in the $\mathfrak{m}$-topology.

Let us see now that Definition 4.3 is independent of the chosen $P$-asymptotic sequence with limit $\hat{f}$. Assume that $\left\{f_{n}\right\}_{n}$ in $\mathcal{O}_{b}(D(\mathbf{0} ; \rho))$ is a $P$-asymptotic sequence, $f \in \mathcal{O}(\Pi), \Pi$ a $P$-sector, such that for all $n \in \mathbb{N}$

$$
\left|f(\boldsymbol{x})-f_{n}(\boldsymbol{x})\right| \leq K_{n}|P(\boldsymbol{x})|^{n},
$$

for $\boldsymbol{x} \in \Pi \cap D(\mathbf{0} ; \rho)$, where $K_{n}>0$ are certain constants.

Let $\left\{\tilde{f}_{n}\right\}_{n}$ be another $P$-asymptotic sequence with $\tilde{f}_{n} \in \mathcal{O}_{b}(D(\mathbf{0} ; \tilde{\rho}))$ and such that $\left\{f_{n}\right\}_{n}$ and $\left\{\tilde{f}_{n}\right\}_{n}$ have the same limit in the $\mathfrak{m}$-adic topology. Without loss of generality we may assume that $\tilde{\rho}=\rho$.

For any given $n \in \mathbb{N}$, we have $J\left(f_{n}\right) \equiv J\left(\tilde{f}_{n}\right) \bmod P^{n} \hat{\mathcal{O}}$. Applying Lemma 2.4 for formal and convergent power series, it follows that actually $J\left(f_{n}\right) \equiv J\left(\tilde{f}_{n}\right) \bmod P^{n} \mathcal{O}$ for all $n$. Applying Lemma 2.6, it follows that there exists some positive $\rho^{\prime}<\rho$ such that for every $n \in \mathbb{N}$ we can write $f_{n}-\tilde{f}_{n}=h_{n} P^{n}$ with some $h_{n} \in \mathcal{O}_{b}\left(D\left(\mathbf{0} ; \rho^{\prime}\right)\right)$.

On $D\left(\mathbf{0} ; \rho^{\prime}\right) \cap \Pi$ we have

$$
\begin{aligned}
\left|f(\boldsymbol{x})-\tilde{f}_{n}(\boldsymbol{x})\right| & \leq\left|f(\boldsymbol{x})-f_{n}(\boldsymbol{x})\right|+\left|h_{n}(\boldsymbol{x})\right||P(\boldsymbol{x})|^{n} \\
& \leq\left(K_{n}+C_{n}\right)|P(\boldsymbol{x})|^{n},
\end{aligned}
$$

where $C_{n}$ denotes some bound of $h_{n}$ on $D\left(\mathbf{0} ; \rho^{\prime}\right)$. This proves that $\left\{\tilde{f}_{n}\right\}_{n}$ also satisfies the inequalities (4.1) and thus can be used to define $f \sim_{\Pi}^{P} \hat{f}$ on $\Pi$.

Contrary to monomial asymptotics, there is no canonical expansion (like in Definition/Proposition 3.11). Using Generalized Weierstrass Division in the form of Lemma 2.6, we are going to present standard expansions in an expression, but they cannot be called canonical, as they depend on the choice of the linear form $\ell$ or equivalently on the choice of the leading monomial of the analytic germ. 
The only case where this expansion is canonical is precisely when this leading monomial does not depend upon the linear form $\ell$, or, in geometric terms, when the Newton polyhedron of $P(\boldsymbol{x})$ has only one vertex. In this case, $P(\boldsymbol{x})=\boldsymbol{x}^{\boldsymbol{\alpha}} U(\boldsymbol{x})$ with some unit $U(\boldsymbol{x})$, and Remark 4.4 (2) reduces the situation to the monomial case.

It is convenient to construct operators $T_{\ell}$ (for injective linear forms $\ell: \mathbb{N}^{d} \rightarrow \mathbb{R}_{+}$) analogous to the operator $T$ used in monomial asymptotics. First we restate Corollaries 2.5 and 2.7 in a slightly different way: $\mathbb{C}$ is replaced by an arbitrary $\mathbb{C}$-vector space $E$. For an injective linear form $\ell: \mathbb{N}^{d} \rightarrow \mathbb{R}_{+}, P \in \mathcal{O} \backslash\{0\}, P(\mathbf{0})=0$, and a vector space $E$, let $\Delta_{\ell}(P, E)$ denote the subset of $E \llbracket \boldsymbol{x} \rrbracket$ defined analogously to (2.4). We abbreviate $\Delta_{\ell}(P)=\Delta_{\ell}(P, \mathbb{C})$.

Lemma 4.6. Let $\ell: \mathbb{N}^{d} \rightarrow \mathbb{R}_{+}$an injective linear form, $P \in \mathcal{O} \backslash\{0\}$, $P(\mathbf{0})=0$. For any vector space $E$, there exists an isomorphism

$$
T_{\ell}: E \llbracket \boldsymbol{x} \rrbracket \rightarrow \Delta_{\ell}(P, E) \llbracket t \rrbracket
$$

with the property $\left(T_{\ell} f\right)(P)=f$ for all series $f \in E \llbracket \boldsymbol{x} \rrbracket$. Here the symbol $\left(T_{\ell} f\right)(P)$ means that $t$ is replaced by $J(P)$ in the series $T_{\ell} f$. If $E$ is a normed vector space and $f \in E\{\boldsymbol{x}\}$ then $T_{\ell} f \in \mathcal{E}_{\ell}\{t\}$, where $\mathcal{E}_{\ell}=\Delta_{\ell}(P, E) \cap E\{\boldsymbol{x}\}$.

For $r>0$ let $\mathcal{E}_{\ell, r}$ denote the Banach space of all functions $f \in$ $\mathcal{O}_{b}(D(\mathbf{0} ; r))$ the series expansion of which $J(f) \in \Delta_{\ell}(P)$. If $r^{\prime}<r$ there is a natural restriction map $\mathcal{E}_{\ell, r} \rightarrow \mathcal{E}_{\ell, r^{\prime}}$, linear and continuous. The image of $f \in \mathcal{E}_{\ell, r}$ will be denoted $\left.f\right|_{\mathcal{E}_{\ell, r^{\prime}}}$. Similarly, if $f(t)=\sum_{n=0}^{\infty} f_{n} t^{n} \in$ $\mathcal{E}_{\ell, r} \llbracket t \rrbracket$ is a formal series, $\left.f(t)\right|_{\mathcal{E}_{\ell, r^{\prime}}}$ will represent $\left.\sum_{n=0}^{\infty} f_{n}\right|_{\mathcal{E}_{\ell, r^{\prime}}} t^{n}$.

In the subsequent theorem, we establish an analogue of the operator $T_{\ell}$ for functions defined on sectors in a germ. This theorem generalizes Lemma 3.8 to arbitrary germs.

Theorem 4.7. Let $\ell: \mathbb{N}^{d} \rightarrow \mathbb{R}_{+}$an injective linear form, $P \in \mathcal{O} \backslash\{0\}$, $P(\mathbf{0})=0$. Let $\Pi=\Pi_{P}(a, b ; \boldsymbol{R})$ a sector in $P$. Then there exists $\rho, \sigma, L>0$ with $P(D(\mathbf{0}, \rho)) \subset D(\mathbf{0}, \sigma)$ and the following properties:

(1) If $f: \Pi \rightarrow \mathbb{C}$ is a holomorphic function on $\Pi$, then there exists a uniquely determined holomorphic function $T_{\ell} f: V(a, b ; \sigma) \times$ $D(\mathbf{0} ; \rho) \rightarrow \mathbb{C}$ such that $J\left(\left(T_{\ell} f\right)(t,).\right) \in \Delta_{\ell}(P)$ for any $t$ and $\left(T_{\ell} f\right)(P(\boldsymbol{x}), \boldsymbol{x})=f(\boldsymbol{x})$ for all $\boldsymbol{x} \in \Pi,|\boldsymbol{x}|<\rho$.

(2) Moreover, given a function $K:] 0, S] \rightarrow \mathbb{R}_{+}, S \geq \sup _{\boldsymbol{x} \in \Pi}|P(\boldsymbol{x})|$, such that $|f(\boldsymbol{x})| \leq K(|P(\boldsymbol{x})|)$ for $\boldsymbol{x} \in \Pi$ we have

$$
\left|\left(T_{\ell} f\right)(t, \boldsymbol{x})\right| \leq \frac{L}{|t|} K(|t|) \quad \text { for } t \in V(a, b, \sigma), \boldsymbol{x} \in D(\mathbf{0} ; \rho) .
$$

Theorem 4.7 will be proved in the next section. 
Remark 4.8. (1) It is important in some applications, that the numbers $\sigma$, $\rho, L$ are independent of the function $f$ to which $T_{\ell}$ is to be applied.

(2) Unfortunately, $T_{\ell} f$ is in general defined on a small set only unlike $T f$ in Lemma 3.8 for monomial asymptotics. As in our theory of asymptotics in a germ, the radius of the sectors or polydisks has to be reduced frequently, this is not crucial. The authors were surprised that such an operator $T_{\ell}$ for asymptotics in a germ exists.

(3) The unicity of $T_{\ell} f$ in statement (1) implies that the operator $T_{\ell}$ is independent of the given $P$-sector in the following sense: If $a \leq a^{\prime}<b^{\prime} \leq b$, $f \in \mathcal{O}_{b}\left(\Pi_{P}(a, b ; \boldsymbol{R})\right), F_{1}=T_{\ell}^{a, b} f: V(a, b, \sigma) \times D(\mathbf{0} ; \rho)$ is the function of statement (1) and $F_{2}=\left.T_{\ell}^{a^{\prime}, b^{\prime}} f\right|_{\Pi_{P}\left(a^{\prime}, b^{\prime} ; \boldsymbol{R}\right)}: V\left(a^{\prime}, b^{\prime}, \sigma^{\prime}\right) \times D\left(\mathbf{0} ; \rho^{\prime}\right)$ is the function of statement (1) for $f$ restricted to the $P$-subsector $\Pi_{P}\left(a^{\prime}, b^{\prime} ; \boldsymbol{R}\right)$, then the restrictions of $F_{1}$ and $F_{2}$ to $V\left(a^{\prime}, b^{\prime}, \tilde{\sigma}\right) \times D(\mathbf{0} ; \tilde{\rho})$, $\tilde{\sigma}=\min \left(\sigma, \sigma^{\prime}\right), \tilde{\rho}=\min \left(\rho, \rho^{\prime}\right)$ coincide. This justifies our notation and will become important later.

The first crucial application of the above theorem generalizes Definition/Proposition 3.11 to asymptotics with respect to an analytic germ.

Theorem 4.9. Let $\ell: \mathbb{N}^{d} \rightarrow \mathbb{R}_{+}$be an injective linear form, $P \in \mathcal{O} \backslash\{0\}$, $P(\mathbf{0})=0$ and let $\Delta(P)$ be defined by (2.4). Let $\Pi$ be a P-sector, $f \in$ $\mathcal{O}(\Pi)$ and $\hat{f} \in \hat{\mathcal{O}}$. Then $f$ has $\hat{f}$ as $P$-asymptotic expansion on $\Pi$ if and only if there exists $\rho>0$ such that $T_{\ell} \hat{f} \in \mathcal{O}_{b}(D(\mathbf{0} ; \rho)) \llbracket t \rrbracket$ and one of the following two equivalent conditions holds:

(1) $T_{\ell} \hat{f}=\sum_{n=0}^{\infty} g_{n}(\boldsymbol{x}) t^{n}$ and for every $N$ there exists $L_{N}>0$ such that $\left|f(\boldsymbol{x})-\sum_{n=0}^{N-1} g_{n}(\boldsymbol{x}) P(\boldsymbol{x})^{n}\right| \leq L_{N}|P(\boldsymbol{x})|^{N} \quad$ for $\boldsymbol{x} \in \Pi \cap D(\mathbf{0} ; \rho)$.

(2) The function $T_{\ell} f$ from Theorem 4.7 is defined on $V(a, b ; \sigma) \times$ $D(\mathbf{0} ; \rho) \rightarrow \mathbb{C}$ for some positive $\sigma$ and satisfies

$$
T_{\ell} f \sim T_{\ell} \hat{f} \quad \text { as } V(a, b ; \sigma) \ni t \rightarrow 0 .
$$

It is worth noting separately that series that are $P$-asymptotic expansions, i.e. $P$-asymptotic series, cannot be arbitrary. The theorem will be proved after the subsequent corollary and several remarks.

Corollary 4.10. If $\hat{f}$ is a $P$-asymptotic series then there exists $\rho>0$ such that $T_{\ell} \hat{f} \in \mathcal{O}_{b}\left(D_{\rho}\right) \llbracket t \rrbracket$, i.e. if $\hat{f}$ is written according to Corollary 2.5

$$
\hat{f}=\sum_{n=0}^{\infty} g_{n} P^{n}, \quad g_{n} \in \Delta_{\ell}(P),
$$

then there exists $\rho>0$ such that for all $n \in \mathbb{N}, g_{n}$ defines an element of $\mathcal{O}_{b}(D(\mathbf{0} ; \rho))$. 
Remark 4.11. (1) The converse is also true. Indeed, $f_{n}=\sum_{k=0}^{n} g_{k} P^{k}$ defines a $P$-asymptotic sequence converging to $\hat{f}$.

(2) The set of the above series is a subset of the completion of $\mathbb{C}\{\boldsymbol{x}\}$ with respect to the valuation defined by the powers of the ideal $(P)$. Observe that their union over all $\rho>0$ does not exhaust the completion: the latter also contains series $\sum_{n=0}^{\infty} g_{n} P^{n}$, where the radii of convergence of the $g_{n}$ tend to 0 .

(3) In the case of a monomial $P=\boldsymbol{x}^{\alpha}$, Theorem 4.9 and Corollary 4.10 confirm that the "new" Definition 4.3 of $\boldsymbol{x}^{\alpha}$-asymptotic expansions is equivalent to the "classical" Definition 3.11 from [CMS].

(4) If $f \in \mathcal{O}_{b}(D(\mathbf{0} ; \rho))$, and $\hat{f}$ is the Taylor expansion of $f$ at the origin, $f$ has $\hat{f}$ as $P$-asymptotic expansion, as $f$ can be written in powers of $P$ by Corollary 2.7 of the Division theorem (Lemma 2.4).

(5) With $\mathcal{E}=\Delta_{\ell}(P) \cap \mathbb{C}\{\boldsymbol{x}\}$, the set $\mathcal{E} \llbracket t \rrbracket$ is not an algebra, as it is not closed under multiplication. Nevertheless, from Definition 4.3 it can be seen that the product of functions having a $P$-asymptotic expansion also has a $P$-asymptotic expansion. Indeed, consider functions $f, g$ on some $P$-asymptotic sector and $P$-asymptotic sequences $\left\{f_{n}\right\}_{n},\left\{g_{n}\right\}_{n}$ satisfying (4.1) corresponding to $P$-asymptotic expansions of $f, g$. Then we can write

$$
f(\boldsymbol{x}) g(\boldsymbol{x})-f_{n}(\boldsymbol{x}) g_{n}(\boldsymbol{x})=f(\boldsymbol{x})\left(g(\boldsymbol{x})-g_{n}(\boldsymbol{x})\right)+\left(f(\boldsymbol{x})-f_{n}(\boldsymbol{x})\right) g_{n}(\boldsymbol{x}) .
$$

So $\left\{f_{n}(\boldsymbol{x}) g_{n}(\boldsymbol{x})\right\}_{n}$ is a $P$-asymptotic sequence converging to $\hat{f}(\boldsymbol{x}) \hat{g}(\boldsymbol{x})$. In fact, if $\left(T_{\ell} \hat{f}\right)(t, \boldsymbol{x})=\sum_{n=0}^{\infty} a_{n}(\boldsymbol{x}) t^{n},\left(T_{\ell} \hat{g}\right)(t, \boldsymbol{x})=\sum_{n=0}^{\infty} b_{n}(\boldsymbol{x}) t^{n}$, and decompose

$$
\sum_{k=0}^{n} a_{k}(\boldsymbol{x}) b_{n-k}(\boldsymbol{x})=\sum_{m=0}^{\infty} h_{n m}(\boldsymbol{x}) P(\boldsymbol{x})^{m},
$$

with $h_{n m} \in \mathcal{E}$, we have

$$
T_{\ell}(\hat{f} \hat{g})(t, \boldsymbol{x})=\sum_{n=0}^{\infty}\left(\sum_{m=0}^{n} h_{n-m, m}(\boldsymbol{x})\right) t^{n} .
$$

(6) It is not evident from Definition 4.3 and the characterization given in Theorem 4.9 that the set of functions having a $P$-asymptotic expansion is stable by partial derivatives. Let $\Pi$ be a $P$-sector, $f \in \mathcal{O}(\Pi)$ having $\hat{f} \in$ $\hat{\mathcal{O}}$ as a $P$-asymptotic expansion. Using the notation of Theorem $4.9(2)$, $T_{\ell} f \sim T_{\ell} \hat{f}$. From the equality $f(\boldsymbol{x})=T_{\ell} f(P(\boldsymbol{x}), \boldsymbol{x})$, we deduce that

$$
\frac{\partial f}{\partial x_{i}}(\boldsymbol{x})=\frac{\partial P}{\partial x_{i}}(\boldsymbol{x}) \frac{\partial\left(T_{\ell} f\right)}{\partial t}(P(\boldsymbol{x}), \boldsymbol{x})+\frac{\partial\left(T_{\ell} f\right)}{\partial x_{i}}(P(\boldsymbol{x}), \boldsymbol{x}) .
$$


As $\frac{\partial\left(T_{\ell} f\right)}{\partial t}(t, \boldsymbol{x})$ and $\frac{\partial\left(T_{\ell} f\right)}{\partial x_{i}}(t, \boldsymbol{x})$ have asymptotic expansion with respect to $t$, by Cauchy's formula, considerations about products made in (3) and (4) imply that $\frac{\partial f}{\partial x_{i}}(\boldsymbol{x})$ has a $P$-asymptotic expansion. Moreover, if we write

$$
\left(T_{\ell} \hat{f}\right)(t, \boldsymbol{x})=\sum_{n=0}^{\infty} f_{n}(\boldsymbol{x}) t^{n},
$$

and expand

$$
\frac{\partial P}{\partial x_{i}}(\boldsymbol{x}) f_{n}(\boldsymbol{x})=\sum_{m=0}^{\infty} g_{n m}(\boldsymbol{x}) P(\boldsymbol{x})^{m},
$$

with $g_{n m}(\boldsymbol{x}) \in \Delta_{\ell}(P)$, then a straightforward computation shows that

$$
T_{\ell}\left(\frac{\partial f}{\partial x_{i}}\right)(t, \boldsymbol{x})=\sum_{n=0}^{\infty}\left(\frac{\partial f_{n}}{\partial x_{i}}(\boldsymbol{x})+\sum_{k=1}^{n+1} k g_{k, n-k+1}(\boldsymbol{x})\right) t^{n} .
$$

Observe that $\frac{\partial f_{n}}{\partial x_{i}}(\boldsymbol{x}) \in \Delta_{\ell}(P)$.

Proof of Theorem 4.9: Assume that $\left\{f_{n}\right\}_{n \in \mathbb{N}}$ is a $P$-asymptotic sequence defined on $D(\mathbf{0} ; R)$ for some positive $R$ satisfying the inequalities (4.1) of Definition 4.3 with the constants $K_{n}$ :

$$
\left|f(\boldsymbol{x})-f_{n}(\boldsymbol{x})\right| \leq K_{n}|P(\boldsymbol{x})|^{n}
$$

for $\boldsymbol{x} \in D(\mathbf{0} ; R) \cap \Pi$, $\Pi$ some $P$-sector, and such that $J\left(f_{n}\right) \equiv \hat{f} \bmod P^{n} \hat{\mathcal{O}}$.

According to Lemma 2.6, we can choose $\mu>0$ such that $D_{\mu} \subset D(\mathbf{0} ; R)$ for the set $D_{\mu}$ of Lemma 2.6; let $Q, R$ denote the operators on $\mathcal{O}_{b}\left(D_{\mu}\right)$ introduced there. Then we can write for all $m \in \mathbb{N}$

$$
f_{n}(\boldsymbol{x})=\sum_{\nu=0}^{m-1} R Q^{\nu}\left(f_{n}\right) P(\boldsymbol{x})^{\nu}+Q^{m}\left(f_{n}\right) P(\boldsymbol{x})^{m} .
$$

As in the proof of Lemma 4.5, we find that $f_{n} \equiv f_{n+1} \bmod P^{n} \mathcal{O}_{b}\left(D_{\mu}\right)$ and hence $R Q^{\nu}\left(f_{n}\right)=R Q^{\nu}\left(f_{m}\right)$, if $\nu<n \leq m$. So, define $g_{n}:=$ $R Q^{n}\left(f_{n+1}\right) \in \mathcal{O}_{b}\left(D_{\mu}\right)$. We have $J\left(g_{n}\right) \in \Delta_{\ell}(P)$ and $g_{n}=R Q^{n}\left(f_{m}\right)$ for all $m>n$.

Then for all $n$,

$$
\begin{aligned}
& \left|f_{n}(\boldsymbol{x})-\sum_{\nu=0}^{n-1} g_{\nu}(\boldsymbol{x}) P^{\nu}(\boldsymbol{x})\right|=\left|f_{n}(\boldsymbol{x})-\sum_{\nu=0}^{n-1} R Q^{\nu}\left(f_{\nu+1}\right) P^{\nu}(\boldsymbol{x})\right| \\
= & \left|f_{n}(\boldsymbol{x})-\sum_{\nu=0}^{n-1} R Q^{\nu}\left(f_{n}\right) P^{\nu}(\boldsymbol{x})\right|=\left|Q^{n}\left(f_{n}\right) P^{n}(\boldsymbol{x})\right| \leq M_{n}|P(\boldsymbol{x})|^{n},
\end{aligned}
$$

for $\boldsymbol{x} \in D_{\mu}$ with some constant $M_{n}$. This first implies that $\hat{f}(\boldsymbol{x})=$ $\sum_{n=0}^{\infty} g_{n}(\boldsymbol{x}) P(\boldsymbol{x})^{n}$ and hence $T_{\ell} \hat{f} \in \mathcal{O}_{b}\left(D_{\mu}\right) \llbracket t \rrbracket$. Together with (4.1), 
this yields

$$
\left|f(\boldsymbol{x})-\sum_{\nu=0}^{n-1} g_{\nu}(\boldsymbol{x}) P^{\nu}(\boldsymbol{x})\right| \leq\left(K_{n}+M_{n}\right)|P(\boldsymbol{x})|^{n}
$$

for $n \in \mathbb{N}$ and $\boldsymbol{x} \in \Pi$. Thus we have proved (1). Application of Theorem 4.7 to $(4.2)$ with $K(s)=\left(K_{n}+M_{n}\right) s^{n}$ yields the existence of some positive $\sigma, \tilde{\rho} \leq \rho$ and $L$ such that

$$
\left|\left(T_{\ell} f\right)(t, \boldsymbol{x})-\sum_{\nu=0}^{n-1} g_{\nu}(\boldsymbol{x}) t^{\nu}\right| \leq L\left(K_{n}+M_{n}\right)|t|^{n-1}
$$

for $(t, \boldsymbol{x}) \in V(a, b ; \sigma) \times D(\mathbf{0} ; \tilde{\rho})$. This proves $(2)$.

The proof of the converses is trivial.

Corollary 4.10 raises the question, whether all formal series $\sum g_{n} P^{n}$, the coefficients $g_{n} \in \mathcal{O}_{b}(D(\mathbf{0} ; \rho))$ of which satisfy $J\left(g_{n}\right) \in \Delta_{\ell}(P)$ can be attained as $P$-asymptotic expansions of some function $f$ on an arbitrary $P$-sector. Using the classical Borel-Ritt Theorem 3.1 (1), it follows easily that this "Borel-Ritt theorem for asymptotics in a germ" is valid. Details are left to the reader.

\section{Proof of Theorem 4.7}

The main problem is to find any function $F$ analytic on $V(a, b ; \sigma) \times$ $D(\mathbf{0} ; \rho)$ satisfying $F(P(\boldsymbol{x}), \boldsymbol{x})=f(\boldsymbol{x})$ for small $\boldsymbol{x}$ in $\Pi_{P}(a, b ; \boldsymbol{R})$ because subsequently Corollary 2.7 can be applied to $F(t,$.$) . In the construc-$ tion of such a function $F$ using induction on $h(P)$, we need to study functions $F$ satisfying $F(P(\boldsymbol{x}), \boldsymbol{x})=0$. This will be done in the two subsequent lemmas.

In this section, we fix a linear form $\ell$ and a germ $P$ as in the hypothesis of the theorem and suppose that $P \in \mathcal{O}(D(\mathbf{0} ; R))$ for some $R>0$. We begin with a simple observation.

Lemma 5.1. Let $a, b, r>0$ and $\mathcal{D} \subset D(\mathbf{0} ; \boldsymbol{R})$ be some domain. For every analytic $F: V(a, b ; r) \times \mathcal{D} \rightarrow \mathbb{C}$ satisfying $F(P(\boldsymbol{x}), \boldsymbol{x})=0$ for $\boldsymbol{x} \in \mathcal{D}$ with $P(\boldsymbol{x}) \in V(a, b ; r)$, there exists a unique analytic function $H: V(a, b ; r) \times \mathcal{D} \rightarrow \mathbb{C}$ such that

$$
F(t, \boldsymbol{x})=(t-P(\boldsymbol{x})) H(t, \boldsymbol{x}) \quad \text { for all } t \in V(a, b ; r), \boldsymbol{x} \in \mathcal{D} .
$$

Proof: $H$ is determined by $H(t, \boldsymbol{x})=F(t, \boldsymbol{x}) /(t-P(\boldsymbol{x}))$ on the set of $(t, \boldsymbol{x})$ with $t \neq P(\boldsymbol{x})$. If there is no $\boldsymbol{x} \in \mathcal{D}$ with $P(\boldsymbol{x}) \in V(a, b ; r)$ then $H$ is obviously analytic on $V(a, b ; r) \times \mathcal{D}$.

If there exists $\boldsymbol{x} \in \mathcal{D}$ such that $P(\boldsymbol{x}) \in V(a, b ; r)$, then the hypothesis implies that $\lim _{t \rightarrow P(\boldsymbol{x})} H(t, \boldsymbol{x})=\frac{\partial F}{\partial t}(P(\boldsymbol{x}), \boldsymbol{x})$ exists. Using Riemann's 
theorem on removable singularities, this shows that, for any fixed $\boldsymbol{x}$, the function $t \mapsto H(t, \boldsymbol{x})$ can be analytically continued to a function holomorphic on $V(a, b ; r)$.

The simplest way to establish analyticity of this continuation with respect to $(t, \boldsymbol{x})$ in the neighborhood of some "critical " point of the form $\left(P\left(\boldsymbol{x}_{0}\right), \boldsymbol{x}_{0}\right) \in V(a, b ; r) \times \mathcal{D}$ is to write

$$
H(t, \boldsymbol{x})=\int_{0}^{1} \frac{\partial F}{\partial t}(\tau t+(1-\tau) P(\boldsymbol{x}), \boldsymbol{x}) d \tau
$$

for all $(t, \boldsymbol{x})$ in its neighborhood.

Lemma 5.2. Let $a, b, r>0$ and $\mathcal{D}, \mathcal{D}^{\prime} \subset \mathbb{C}^{d}$ be two domains such that the closure of $\mathcal{D}^{\prime}$ is compact and contained in $\mathcal{D}$. Then there exists $L>0$ with the following property: For every analytic $F: V(a, b ; r) \times$ $\mathcal{D} \rightarrow \mathbb{C}$ satisfying $F(P(\boldsymbol{x}), \boldsymbol{x})=0$ for $\boldsymbol{x} \in \mathcal{D}$ with $P(\boldsymbol{x}) \in V(a, b ; r)$ and $\sup _{\boldsymbol{x} \in \mathcal{D}}|F(t, \boldsymbol{x})| \leq K(t)$ with some $K: V(a, b ; r) \rightarrow \mathbb{R}_{+}$, the unique analytic function $H: V(a, b ; r) \times \mathcal{D} \rightarrow \mathbb{C}$ of Lemma 5.1 with (5.1) satisfies

$$
\sup _{\boldsymbol{x} \in \mathcal{D}^{\prime}}|H(t, \boldsymbol{x})| \leq L K(t) \quad \text { for } t \in V(a, b ; r) .
$$

Remark 5.3. Lemma 5.1 implies that $t-P(\boldsymbol{x})$ divides $F(t, \boldsymbol{x})$. Therefore Lemma 2.3 could be applied to these functions of $(t, \boldsymbol{x})$. Unfortunately this does not yield the desired result as we would have estimates for $H$ only on $(t, \boldsymbol{x})$-subsets compactly contained in $V(a, b, r) \times \mathcal{D}$ which cannot have points with $t=0$ on their boundary.

Proof: It is close to that of Lemma 2.3, but use of Lemma 2.6 in this special situation improves the domains of validity of the estimates.

By a classical argument of compactness, it is sufficient to prove that for every $t_{0}$ in the closure of $V(a, b, r)$ and every $\boldsymbol{x}_{0} \in \mathcal{D}$, there exist $\delta, L>0$ and a neighborhood $\mathcal{U} \subset \mathcal{D}$ of $\boldsymbol{x}_{0}$ such that for every function $F: V(a, b ; r) \times \mathcal{D} \rightarrow \mathbb{C}$ fulfilling the hypothesis of the theorem with some majorant $K$, the quotient $H$ from the previous lemma satisfies

$$
\sup _{\boldsymbol{x} \in \mathcal{U}}|H(t, \boldsymbol{x})| \leq L K(t) \quad \text { for } t \in V(a, b ; r) \cap D\left(t_{0}, \delta\right) .
$$

For the proof of this statement, we have to distinguish two cases.

If $P\left(\boldsymbol{x}_{0}\right) \neq t_{0}$, then $|t-P(\boldsymbol{x})|$ is bounded below by some positive constant if $t$ is sufficiently close to $t_{0}$ and $\boldsymbol{x}$ sufficiently close to $\boldsymbol{x}_{0}$. In this case, the existence of $\delta, L, \mathcal{U}$ is immediate.

If $P\left(\boldsymbol{x}_{0}\right)=t_{0}$, then we apply the results of Subsection 2.3 to $\tilde{P}(\boldsymbol{x})=$ $P\left(\boldsymbol{x}_{0}+\boldsymbol{x}\right)-t_{0}$. We choose some injective linear form $\tilde{\ell}$ and define $\Delta_{\tilde{\ell}}(\tilde{P})$ accordingly (see (2.4)). We choose some neighborhood $\tilde{\mathcal{U}}$ of $\mathbf{0}$ such that Lemma 2.6 can be applied. This yields bounded linear operators $\tilde{Q}, \tilde{R}: \mathcal{O}_{b}(\mathcal{U}) \rightarrow \mathcal{O}_{b}(\mathcal{U}), \mathcal{U}:=\boldsymbol{x}_{0}+\tilde{\mathcal{U}}$, such that for all functions $g, q, r \in$ 
$\mathcal{O}_{b}(\mathcal{U})$, we have $g=\left(P-t_{0}\right) q+r, J_{\boldsymbol{x}_{0}}(r) \in \Delta_{\tilde{\ell}}(\tilde{P})$ if and only if $q=\tilde{Q}(g)$ and $r=\tilde{R}(g)$. Here $J_{\boldsymbol{x}_{0}}(r)$ denotes the Taylor expansion of the function $\boldsymbol{x} \rightarrow r\left(\boldsymbol{x}_{0}+\boldsymbol{x}\right)$.

Equation (5.1) is equivalent to

$$
\left(t-t_{0}\right) H(t, \boldsymbol{x})-F(t, \boldsymbol{x})=\left(P(\boldsymbol{x})-t_{0}\right) H(t, \boldsymbol{x})+0
$$

for all $(t, \boldsymbol{x})$. Thus for $t \in V(a, b, r)$, the functions $h, f: \mathcal{U} \rightarrow \mathbb{C}$ defined by $h(\boldsymbol{x})=H(t, \boldsymbol{x}), f(\boldsymbol{x})=F(t, \boldsymbol{x})$ for $\boldsymbol{x} \in \mathcal{U}$ satisfy

$$
h=\left(t-t_{0}\right) \tilde{Q}(h)-\tilde{Q}(f),
$$

with the above operator $\tilde{Q}$ on $\mathcal{O}_{b}(\mathcal{U})$. If $\delta>0$ is sufficiently small, the fixed point principle can be applied to (5.2) if $\left|t-t_{0}\right|<\delta$ and yields that $\|h\| \leq \frac{\|\tilde{Q}\|}{1-\delta\|\tilde{Q}\|}\|f\|$, where $\|\cdot\|$ denotes the maximum norm. This yields

$$
\sup _{\boldsymbol{x} \in \mathcal{U}}|H(t, \boldsymbol{x})| \leq \frac{\|\tilde{Q}\|}{1-\delta\|\tilde{Q}\|} \sup _{\boldsymbol{x} \in \mathcal{U}}|F(t, \boldsymbol{x})|
$$

if $t \in V(a, b, r),\left|t-t_{0}\right|<\delta$. Hence we can choose the above $\delta, L=\frac{\|\tilde{Q}\|}{1-\delta\|\tilde{Q}\|}$ and the above neighborhood $\mathcal{U}$ of $\boldsymbol{x}_{0}$ to obtain the wanted statement. This completes the proof.

The main step is:

Lemma 5.4. Let $P \in \mathcal{O} \backslash\{0\}, P(\mathbf{0})=0$ and let $\Pi=\Pi_{P}(a, b ; \boldsymbol{R}) a$ sector in $P$. Then there exist $\rho, \sigma, L>0$ with $P(D(\mathbf{0} ; \rho)) \subset D(\mathbf{0} ; \sigma)$ and the following properties:

(1) If $f: \Pi \rightarrow \mathbb{C}$ is a holomorphic function, then there exists a holomorphic function $F: V(a, b ; \sigma) \times D(\mathbf{0} ; \rho) \rightarrow \mathbb{C}$ such that $F(P(\boldsymbol{x}), \boldsymbol{x})=$ $f(\boldsymbol{x})$ for all $\boldsymbol{x} \in \Pi,|\boldsymbol{x}|<\rho$.

(2) Moreover, given a function $K:] 0, S] \rightarrow \mathbb{R}_{+}, S \geq \sup _{\boldsymbol{x} \in \Pi}|P(\boldsymbol{x})|$, such that $|f(\boldsymbol{x})| \leq K(|P(\boldsymbol{x})|)$ for $\boldsymbol{x} \in \Pi$, the function $F$ of statement (1) satisfies

$$
|F(t, \boldsymbol{x})| \leq \frac{L}{|t|} K(|t|) \quad \text { for } t \in V(a, b ; \sigma), \boldsymbol{x} \in D(\mathbf{0} ; \rho) .
$$

Proof: The statements can be formally combined if we allow a function $K$ with $K(s) \equiv \infty$. Thus we prove both statements together by induction on $h(P)$ using Lemma 2.1.

If $h(P)=0$ then $P$ has normal crossings and the statement can be reduced to the monomial version, where Lemma 3.8 even gives a better result. Assume now that the statement is true whenever $h(Q) \leq m$ and prove it if $h(P)=m+1$. As the statement does not change by right composition of $P$ with a diffeomorphism, we can assume that $h\left(P \circ b_{\xi}\right) \leq$ $m$ for all $\xi \in \mathbb{P}_{\mathbb{C}}^{1}$ or $h\left(P \circ r_{k}\right) \leq m$ for some $k \in \mathbb{N}$. See Subsection 2.2 for notation. 
We first assume that the statement is true for all $P \circ b_{\xi}, \xi \in \mathbb{P}_{\mathbb{C}}^{1}$. Then for every $\xi \in \mathbb{P}_{\mathbb{C}}^{1}$ and every sector $\Pi_{\xi}:=\Pi_{P \circ b_{\xi}}(a, b ; \tilde{\boldsymbol{R}})$, there exist $\rho_{\xi}, \sigma_{\xi}, L_{\xi}>0$ such that for every holomorphic $\tilde{f}: \Pi_{\xi} \rightarrow \mathbb{C}$ and $\left.K:] 0, \sigma_{\xi}\right] \rightarrow \mathbb{R}_{+} \cup\{\infty\}$ with $|\tilde{f}(\boldsymbol{z})| \leq K\left(\left|\left(P \circ b_{\xi}\right)(\boldsymbol{z})\right|\right)$ for $\boldsymbol{z} \in \Pi_{\xi}$ there exists a holomorphic $\tilde{F}: V\left(a, b ; \sigma_{\xi}\right) \times D\left(\mathbf{0} ; \rho_{\xi}\right) \rightarrow \mathbb{C}$ with

$$
\tilde{f}(\boldsymbol{z})=\tilde{F}\left(\left(P \circ b_{\xi}\right)(\boldsymbol{z}), \boldsymbol{z}\right) \text { for } \boldsymbol{z} \in \Pi_{\xi} \cap D\left(\mathbf{0}, \rho_{\xi}\right)
$$

and $|\tilde{F}(t, \boldsymbol{z})| \leq \frac{L_{\xi}}{|t|} K(|t|)$ for $(t, \boldsymbol{z}) \in V\left(a, b ; \sigma_{\xi}\right) \times D\left(\mathbf{0} ; \rho_{\xi}\right)$.

Given some analytic $f: \Pi \rightarrow \mathbb{C}$ and $K:] 0, S] \rightarrow \mathbb{R}_{+} \cup\{\infty\}$ with $|f(\boldsymbol{x})| \leq K(|P(\boldsymbol{x})|)$ for $\boldsymbol{x} \in \Pi$, let $F_{\xi}$ denote the holomorphic function on $V\left(a, b ; \sigma_{\xi}\right) \times D\left(\mathbf{0} ; \rho_{\xi}\right)$ corresponding to $\tilde{f}=f \circ b_{\xi}$. As before we use $\phi_{\xi}$ to carry over these statements to neighborhoods of points of the exceptional divisor. So define $G_{\xi}$ on $V\left(a, b ; \sigma_{\xi}\right) \times U_{\xi}, U_{\xi}=\phi_{\xi}^{-1}\left(D\left(\mathbf{0}, \rho_{\xi}\right)\right)$ such that $G_{\xi}(t, p)=F_{\xi}\left(t, \phi_{\xi}(p)\right)$. By construction, we have

$$
G_{\xi}((P \circ b)(p), p)=(f \circ b)(p) \quad \text { for } p \in U_{\xi}, \phi_{\xi}(p) \in \Pi_{\xi}
$$

and $\left|G_{\xi}(t, p)\right| \leq \frac{L_{\xi}}{|t|} K(|t|)$ for $(t, p) \in V\left(a, b, \sigma_{\xi}\right) \times U_{\xi}$. The differences $D_{\xi \eta}(t, p):=G_{\xi}(t, p)-G_{\eta}(t, p)$ are then defined and holomorphic for $t \in V\left(a, b ; \sigma_{\xi \eta}\right), \sigma_{\xi \eta}:=\min \left(\sigma_{\xi}, \sigma_{\eta}\right)$, and $p \in U_{\xi} \cap U_{\eta}$. They satisfy

$$
\left.D_{\xi \eta}((P \circ b)(p), p)=0 \quad \text { for small } p \in U_{\xi} \cap U_{\eta}, \arg (P(b(p))) \in\right] a, b[
$$

and $\left|D_{\xi \eta}(t, p)\right| \leq \frac{L_{\xi}+L_{\eta}}{|t|} K(|t|)$ for $\left.t \in V\left(a, b ; \sigma_{\xi \eta}\right)\right), p \in U_{\xi} \cap U_{\eta}$.

In order to apply Lemma 5.2 (resp. Lemma 5.1 in the case $K(s) \equiv \infty$ ), we also consider $\hat{U}_{\xi}=\phi_{\xi}^{-1}\left(D\left(0 ; \hat{r}_{\xi}\right)\right)$ with some positive $\hat{r}_{\xi}<r_{\xi}$. Then this lemma, applied to $D_{\xi \eta}$ - more precisely to their right composition with $\phi_{\xi}$ - yields holomorphic functions $Q_{\xi \eta}: V\left(a, b ; \sigma_{\xi \eta}\right) \times\left(\hat{U}_{\xi} \cap \hat{U}_{\eta}\right) \rightarrow \mathbb{C}$ satisfying

$$
D_{\xi \eta}(t, p)=(t-(P \circ b)(p)) Q_{\xi \eta}(t, p)
$$

and $\left|Q_{\xi \eta}(t, p)\right| \leq C_{\xi \eta}\left(L_{\xi}+L_{\eta}\right) \frac{1}{|t|} K(|t|)$ on the domain of $Q_{\xi \eta}$ with some constant $C_{\xi \eta}$ depending only upon $a, b, \sigma_{\xi \eta}, U_{\xi} \cap U_{\eta}$, and $\hat{U}_{\xi} \cap \hat{U}_{\eta}$.

As the $\hat{U}_{\xi}, \xi \in \mathbb{P}_{\mathbb{C}}^{1}$ cover the exceptional divisor in the blow-up variety $M$, there exists a finite subcover, say corresponding to $\xi_{j}, j=$ $0, \ldots, N$. We now apply Lemma 2.10 and Remark 2.11 to the collection $\left(Q_{\xi_{j} \xi_{k}}\right)_{j, k=0, \ldots, N}$ of holomorphic functions $Q_{\xi_{j} \xi_{k}}: V(a, b ; \tilde{\sigma}) \times\left(\hat{U}_{\xi_{j}} \cap\right.$ $\left.\hat{U}_{\xi_{k}}\right) \rightarrow \mathbb{C}, j, k=0, \ldots, N, \tilde{\sigma}$ the minimum of $\sigma_{\xi_{j}}, j=0, \ldots, N$. We obtain a collection of holomorphic functions $R_{\xi_{j}}: V(a, b ; \tilde{\sigma}) \times \tilde{U}_{\xi_{j}} \rightarrow \mathbb{C}$, satisfying

$$
Q_{\xi_{i}, \xi_{j}}(t, p)=R_{\xi_{i}}(t, p)-R_{\xi_{j}}(t, p) \quad \text { for }(t, p) \in V(a, b ; \tilde{\sigma}) \times\left(\tilde{U}_{\xi_{i}} \cap \tilde{U}_{\xi_{j}}\right)
$$


and $\sup _{p \in \tilde{U}_{\xi_{j}}}\left|R_{\xi_{j}}(t, p)\right| \leq \frac{\tilde{C}}{|t|} K(|t|)$ for $t \in V(a, b, \tilde{\sigma})$. Here $\tilde{U}_{\xi_{j}} \subset$ $M$ are the open subsets of $U_{\xi_{j}}$ in Lemma 2.10 covering $E$ and with the constant $C$ from Lemma 2.10 , the constant $\tilde{C}$ is the maximum of $C C_{\xi_{j} \xi_{k}}\left(L_{\xi_{j}}+L_{\xi_{k}}\right), j, k=0, \ldots, N$.

Now we can define holomorphic functions $\tilde{G}_{\xi_{j}}: V(a, b ; \tilde{\sigma}) \times \tilde{U}_{\xi_{j}} \rightarrow \mathbb{C}$, $j=0, \ldots, N$ by

$$
\tilde{G}_{\xi_{j}}(t, p)=G_{\xi_{j}}(t, p)-(t-(P \circ b)(p)) R_{\xi_{j}}(t, p) .
$$

By the construction of $R_{\xi_{j}}$, the family $\tilde{G}_{\xi_{j}}$ glues together, i.e. $\tilde{G}_{\xi_{i}}(t, p)=$ $\tilde{G}_{\xi_{j}}(t, p)$ whenever $p \in \tilde{U}_{\xi_{i}} \cap \tilde{U}_{\xi_{j}}$. As at the end of the proof of Lemma 2.2, this implies that there exists a positive $\bar{\rho}$ and a holomorphic function $F: V(a, b ; \tilde{\sigma}) \times D(\mathbf{0}, \bar{\rho}) \rightarrow \mathbb{C}$ such that $F(t, b(p))=\tilde{G}_{\xi_{j}}(t, p)$ for $t \in$ $V(a, b ; \tilde{\sigma})$ and $p \in \tilde{U}_{\xi_{j}}$ with $b(p) \in D(\mathbf{0}, \bar{\rho})$. We can assume without loss of generality that $P(D(\mathbf{0} ; \bar{\rho})) \subset D(\mathbf{0} ; \tilde{\sigma})$. An easy calculation shows that $F(P(\boldsymbol{x}), \boldsymbol{x})=f(\boldsymbol{x})$ for $\boldsymbol{x} \in \Pi_{P}(a, b ; \bar{\rho})$.

By their definition, we have $\sup _{p \in \tilde{U}_{\xi_{j}}}\left|\tilde{G}_{\xi_{j}}(t, p)\right| \leq\left(L_{\xi_{j}}+2 \tilde{\sigma} \tilde{C}\right) \frac{1}{|t|} K(|t|)$ for $j=0, \ldots, N$ and hence

$$
\sup _{\boldsymbol{x} \in D(0 ; \bar{\rho})}|F(t, \boldsymbol{x})| \leq\left(\max _{j=0, \ldots, N} L_{\xi_{j}}+2 \tilde{\sigma} \tilde{C}\right) \frac{1}{|t|} K(|t|)
$$

for $t \in V(a, b ; \tilde{\sigma})$. This completes the proof of the lemma in the case of blow-ups.

The case of a ramification is much simpler and left to the reader.

Now we are in a position to prove Theorem 4.7, combining the two statements as in the above proof of Lemma 5.4. This lemma provides positive $\tilde{\rho}, \tilde{\sigma}, \tilde{L}$ and, for given holomorphic $f: \Pi \rightarrow \mathbb{C}$ with an estimate $|f(\boldsymbol{x})| \leq K(|P(\boldsymbol{x})|)$ on $\Pi$, it yields a holomorphic function $F: V(a, b ; \tilde{\sigma}) \times D(\mathbf{0} ; \tilde{\rho}) \rightarrow \mathbb{C}$ satisfying $F(P(\boldsymbol{x}), \boldsymbol{x})=f(\boldsymbol{x})$ for $\boldsymbol{x} \in D(\mathbf{0} ; \tilde{\rho})$ and $|F(t, \boldsymbol{x})| \leq \frac{\tilde{L}}{|t|} K(|t|)$ on $V(a, b ; \tilde{\sigma}) \times D(\mathbf{0} ; \tilde{\rho})$. In order to apply Lemma 2.6, we restrict $F$ to $V(a, b ; \tilde{\sigma}) \times D_{s}$, where $D_{s} \subset D(\mathbf{0} ; \tilde{\rho})$ is chosen such that the operators $Q, R$ of Lemma 2.6 are defined.

As in the proof of Corollary 2.7, we can write

$$
F(t, \boldsymbol{x})=\sum_{n=0}^{N-1}\left(\left(R Q^{n}\right)(F(t, .))\right)(\boldsymbol{x}) P(\boldsymbol{x})^{n}+\left(Q^{N} F(t, .)\right)(\boldsymbol{x}) P(\boldsymbol{x})^{N}
$$

for $t \in V(a, b ; \tilde{\sigma}), N \in \mathbb{N}$ and $\boldsymbol{x} \in D_{s}$ by repeated application of Lemma 2.6. If $\rho>0$ is so small that $D(\mathbf{0} ; \rho) \subset D_{s}$ and $B=\sup \{|P(\boldsymbol{x})| ; \boldsymbol{x} \in D(\mathbf{0} ; \rho)\}<$ $\frac{1}{\|Q\|}$ then $\left|\left(Q^{N} F(t,).\right)(\boldsymbol{x}) P(\boldsymbol{x})^{N}\right| \leq(B\|Q\|)^{N}\|F(t,).\| \rightarrow 0$ as $N \rightarrow \infty$ for $\boldsymbol{x} \in D(\mathbf{0}, \rho)$ and we obtain $F(t, \boldsymbol{x})=\sum_{n=0}^{\infty}\left(\left(R Q^{n}\right)(F(t,)).\right)(\boldsymbol{x}) P(\boldsymbol{x})^{n}$ 
for these $\boldsymbol{x}$. Now we define the desired function $T_{\ell} f$ by

$$
\left(T_{\ell} f\right)(t, \boldsymbol{x})=\sum_{n=0}^{\infty}\left(\left(R Q^{n}\right)(F(t, .))\right)(\boldsymbol{x}) t^{n}
$$

for $t \in V(a, b ; \sigma), \boldsymbol{x} \in D(\mathbf{0} ; \rho)$; here $\sigma=\min (\tilde{\sigma}, B)$ and we reduce $\rho$ if necessary so that $P(D(\mathbf{0} ; \rho)) \subset D(\mathbf{0} ; \sigma)$. The function $T_{\ell} f$ is independent of the choice of $F$ in Lemma 5.4. This follows from the uniqueness established at the end of this proof. Note also that the choice of $F$ does not depend on the linear form $\ell$. By construction, we then have $\left(T_{\ell} f\right)(P(\boldsymbol{x}), \boldsymbol{x})=F(P(\boldsymbol{x}), \boldsymbol{x})=f(\boldsymbol{x})$ for $\boldsymbol{x} \in \Pi_{P}(a, b, \rho)$. As

$$
\sup _{\boldsymbol{x} \in D(\mathbf{0}, \rho)}\left|\left(\left(R Q^{n}\right)(F(t, .))\right)(\boldsymbol{x})\right| \leq\|R\|\|Q\|^{n} \sup _{\boldsymbol{x} \in D(\mathbf{0}, \tilde{\rho})}|F(t, \boldsymbol{x})|,
$$

we obtain that

$$
\sup _{\boldsymbol{x} \in D(\mathbf{0}, \rho)}\left|\left(T_{\ell} f\right)\right|(t, \boldsymbol{x}) \leq \frac{\|R\|}{1-B\|Q\|} \sup _{\boldsymbol{x} \in D(\mathbf{0}, \tilde{\rho})}|F(t, \boldsymbol{x})| \leq \frac{L}{|t|} K(|t|)
$$

for $t \in V(a, b, \sigma)$, where $L=\frac{\tilde{L}\|R\|}{1-B\|Q\|}$. By the definition of $R$, the expansion $J\left(\left(R Q^{n}\right)(F(t,)\right.$.$) is in \Delta_{\ell}(P)$ for any $t, n$. Hence also $J\left(\left(T_{\ell} f\right)(t,).\right) \in$ $\Delta_{\ell}(P)$ for $t \in V(a, b, \sigma)$ as desired. Hence the function $T_{\ell} f$ satisfies the properties wanted in the theorem. It is defined and holomorphic on $V(a, b, \sigma) \times D(\mathbf{0}, \rho)$ and the above construction of $\sigma, \rho, L$ is independent of $f$.

Thus it remains to show the uniqueness of $T_{\ell} f$. If $G: V(a, b, \sigma) \times$ $D(\mathbf{0}, \rho) \rightarrow \mathbb{C}$ is another holomorphic function satisfying $G(P(\boldsymbol{x}), \boldsymbol{x})=$ $f(\boldsymbol{x})$ for sufficiently small $\boldsymbol{x} \in \Pi_{P}(a, b, R)$ and $J(G(t,).) \in \Delta_{\ell}(P)$ for $t \in V(a, b, \sigma)$, then $\delta=T_{\ell} f-G: V(a, b, \sigma) \times D(\mathbf{0}, \rho) \rightarrow \mathbb{C}$ satisfies

$$
\begin{array}{ll}
J(\delta(t, .)) \in \Delta_{\ell}(P) & \text { for } t \in V(a, b, \sigma), \text { and } \\
\delta(P(\boldsymbol{x}), \boldsymbol{x})=0 & \text { for small } \boldsymbol{x} \in \Pi_{P}(a, b, \mu), \text { if } \mu>0 \text { is small enough. }
\end{array}
$$

We will show that this implies $\delta=0$.

Indeed, let $H: V(a, b, \sigma) \times D(\mathbf{0}, \rho) \rightarrow \mathbb{C}$ denote the function of Lemma 5.1 with

$$
\delta(t, \boldsymbol{x})=(t-P(\boldsymbol{x})) H(t, \boldsymbol{x})
$$

for all $t, \boldsymbol{x}$. For sufficiently small positive $s$, the operators $Q, R$ of Lemma 2.6 are defined on $\mathcal{O}_{b}\left(D_{s}\right)$ and the restriction $h_{t}$ of $H(t,$.$) to D_{s}$ is bounded, i.e. in $\mathcal{O}_{b}\left(D_{s}\right)$. For each fixed $t$, we can apply $Q$ to equation (5.3) and obtain

$$
h_{t}=t Q\left(h_{t}\right)
$$

because $J(\delta(t,).) \in \Delta_{\ell}(P)$ implies $Q\left(\left.\delta(t,)\right|_{.D_{s}}\right)=0$. As $Q$ is a bounded linear operator on $\mathcal{O}_{b}\left(D_{s}\right)$, this is only possible if $h_{t}=0$, provided $t$ is sufficiently small. This means that $H(t, \boldsymbol{x})=0$ for all sufficiently small $t$ and all $\boldsymbol{x} \in D_{s}$. By the identity theorem, $H$ must vanish and hence also $\delta$. This proves that $G=T_{\ell} f$ and thus the last assertion of the theorem. 


\section{Behaviour under blow-ups and ramification}

In this section, we study how the notion of asymptotics with respect to an analytic germ behaves under blow-ups and ramification. This will be useful to reduce the notion to monomial asymptotics when necessary. The statements and proofs of this section also prepare analogous ones in Section 7.

Consider a nonzero germ $P \in \mathcal{O}=\mathbb{C}\left\{x_{1}, \ldots, x_{d}\right\}$, not a unit, and suppose it is defined on $D(\mathbf{0} ; R)$. All radii of polydisks in this section are assumed to be smaller than $R$, but we will not mention this below. Consider some $P$-sector $\Pi=\Pi_{P}(\alpha, \beta ; \rho)$ and a function $f$ holomorphic on $\Pi$.

If we suppose that some $\hat{f}$ is the $P$-asymptotic expansion of $f$ on $\Pi$, then it is straightforward that $\hat{f} \circ b_{\xi}$ is the $\left(P \circ b_{\xi}\right)$-asymptotic expansion of $f \circ b_{\xi}$ on any $\left(P \circ b_{\xi}\right)$-sector $\Pi_{P \circ b_{\xi}}(\alpha, \beta ; r)$ with sufficiently small $r>0$. The converse is much more interesting, also for applications.

Proposition 6.1. Consider $P$ on $D(\mathbf{0} ; \rho), \Pi=\Pi_{P}(\alpha, \beta ; \rho)$, and $f: \Pi \rightarrow$ $\mathbb{C}$ holomorphic as above. Suppose that for every $\xi \in \mathbb{P}_{\mathbb{C}}^{1}$, the function $f \circ$ $b_{\xi}$, restricted to $\Pi_{\xi}=\Pi_{P \circ b_{\xi}}\left(\alpha, \beta ; r_{\xi}\right)$ with some sufficiently small $r_{\xi}$, has some formal series $\hat{g}_{\xi} \in \hat{\mathcal{O}}$ as $\left(P \circ b_{\xi}\right)$-asymptotic expansion on its domain.

Then there exists a formal series $\hat{f} \in \hat{\mathcal{O}}$ that is the P-asymptotic expansion of $f$ on $\Pi$ and it satisfies $\hat{f} \circ b_{\xi}=\hat{g}_{\xi}$ for all $\xi \in \mathbb{P}_{\mathbb{C}}^{1}$.

Proof: Using Definition 4.3, we can assume that, for every $\xi \in \mathbb{P}_{\mathbb{C}}^{1}$, there are sequences $\left\{g_{n}^{(\xi)}\right\}_{n}$ of bounded holomorphic functions $g_{n}^{(\xi)}: D\left(\mathbf{0} ; r_{\xi}\right) \rightarrow$ $\mathbb{C}$ with $J\left(g_{n}^{(\xi)}\right) \rightarrow \hat{g}_{\xi}$ as $n \rightarrow \infty$ and positive constants $C_{n}^{(\xi)}$ such that

$$
\left|\left(f \circ b_{\xi}\right)(\mathbf{v})-g_{n}^{(\xi)}(\mathbf{v})\right| \leq C_{n}^{(\xi)}\left|\left(P \circ b_{\xi}\right)(\mathbf{v})\right|^{n}
$$

for $n \in \mathbb{N}$ and $\mathbf{v} \in \Pi_{\xi}$.

As in the proof of Lemma 2.2, we consider the neighborhoods $U_{\xi}=$ $\phi_{\xi}^{-1}\left(D\left(0 ; r_{\xi}\right)\right)$ of $(\xi, \mathbf{0})$ in $M$ and the functions $G_{n}^{(\xi)}=g_{n}^{(\xi)} \circ \phi_{\xi}$ holomorphic on $U_{\xi}$. In order to apply Lemma 2.3 at some point, we also consider $\tilde{U}_{\xi}=\phi_{\xi}^{-1}\left(D\left(0 ; \tilde{r}_{\xi}\right)\right)$ with some positive $\tilde{r}_{\xi}<r_{\xi}$.

By (6.1), we have

$$
\left|G_{n}^{(\xi)}-G_{n}^{(\zeta)}\right|(p) \leq\left(C_{n}^{(\xi)}+C_{n}^{(\zeta)}\right)|P(b(p))|^{n}
$$

for $\xi, \zeta \in \mathbb{P}_{\mathbb{C}}^{1}, p \in U_{\xi} \cap U_{\zeta}$ with $\alpha<\arg (P(b(p)))<\beta$. Now we apply Lemma 2.3 - more precisely after right composition with $\phi_{\xi}$ - and obtain 
that there are bounded holomorphic functions $H_{n}^{(\xi, \zeta)}: \tilde{U}_{\xi} \cap \tilde{U}_{\zeta} \rightarrow \mathbb{C}$ such that

$$
G_{n}^{(\xi)}-G_{n}^{(\zeta)}=H_{n}^{(\xi, \zeta)}(P \circ b)^{n} .
$$

More precisely, we have $\left\|H_{n}^{(\xi, \zeta)}\right\| \leq L^{(\xi, \zeta)}\left(C_{n}^{(\xi)}+C_{n}^{(\zeta)}\right)$, where $L^{(\xi, \zeta)}$ denotes the constant of Lemma 2.3 for the domains $U_{\xi} \cap U_{\zeta}$ and $\tilde{U}_{\xi} \cap \tilde{U}_{\zeta}$, and, as usual, $\|\cdot\|$ denotes the maximum norm.

Now the neighborhoods $\tilde{U}_{\xi}, \xi \in \mathbb{P}_{\mathbb{C}}^{1}$ of $(\xi, \mathbf{0})$ cover the compact set $E=\mathbb{P}_{\mathbb{C}}^{1} \times\{\mathbf{0}\}$ and therefore there is a finite subcover, say corresponding to $\xi_{0}, \ldots, \xi_{K}$.

At this point, Lemma 2.10 can be applied. It yields open sets $\bar{U}_{\xi_{j}} \subset$ $\tilde{U}_{\xi_{j}}$ forming an open cover of $E$ and a constant $C$ used later in the estimates. For $n \in \mathbb{N}$, we now apply it to the family $H_{n}^{\left(\xi_{i}, \xi_{j}\right)}, i, j=$ $0, \ldots, K$, and obtain bounded holomorphic functions $L_{n}^{\left(\xi_{j}\right)}: \bar{U}_{\xi_{j}} \rightarrow \mathbb{C}$, $j=0, \ldots, K$ satisfying

$$
\left\|L_{n}^{\left(\xi_{j}\right)}\right\|_{\infty} \leq C \max \left\{\left\|H_{n}^{\left(\xi_{\ell}, \xi_{k}\right)}\right\|_{\infty} \mid \ell, k \in\{0, \ldots, K\}\right\}
$$

and $H_{n}^{\left(\xi_{j}, \xi_{k}\right)}=L_{n}^{\left(\xi_{j}\right)}-L_{n}^{\left(\xi_{k}\right)}$ for $j, k=0, \ldots, K$.

With these functions $L_{n}^{\left(\xi_{j}\right)}$ we now define

$$
F_{n}^{\left(\xi_{j}\right)}=G_{n}^{\left(\xi_{j}\right)}-L_{n}^{\left(\xi_{j}\right)}(P \circ b)^{n}
$$

for $j=0, \ldots, K, n \in \mathbb{N}$. Then $F_{n}^{\left(\xi_{j}\right)}$ are defined on $\bar{U}_{\xi_{j}}$ for $j=0, \ldots, K$. The domains of the functions $F_{n}^{\left(\xi_{j}\right)}, j=0, \ldots, K$ again cover a neighborhood $\tilde{V}$ of the exceptional divisor $E$ in $M$. By construction, the functions satisfy

$$
\left|f(b(p))-F_{n}^{\left(\xi_{j}\right)}(p)\right| \leq\left(C_{n}^{\left(\xi_{j}\right)}+\left\|L_{n}^{\left(\xi_{j}\right)}\right\|_{\infty}\right)|P(b(p))|^{n}
$$

for $n \in \mathbb{N}, j=1, \ldots, K$ and $p$ in the domain of $F_{n}^{\left(\xi_{j}\right)}, \alpha<\arg (P(b(p)))<$ $\beta$. Again by construction, the functions $F_{n}^{\left(\xi_{j}\right)}, j=0, \ldots, K$ coincide on the intersections of their domains, i.e. they glue together to functions $F_{n}: \tilde{V} \rightarrow \mathbb{C}$. As in the proof of Lemma 2.2, this implies that there are holomorphic bounded functions $f_{n}: V \rightarrow \mathbb{C}, n \in \mathbb{N}$, defined on the neighborhood $V=b(\tilde{V})$ of $\mathbf{0}$ in $\mathbb{C}^{d}$ such that $F_{n}=f_{n} \circ b$ on $\tilde{V}$. The inequalities (6.4) yield that

$$
\left|f(\boldsymbol{x})-f_{n}(\boldsymbol{x})\right| \leq B_{n}|P(x)|^{n} \quad \text { for } \boldsymbol{x} \in V, \alpha<\arg (P(\boldsymbol{x}))<\beta
$$

i.e. on $V \cap \Pi$, where $B_{n}$ is the maximum of the above constants $C_{n}^{\left(\xi_{j}\right)}+$ $\left\|L_{n}^{\left(\xi_{j}\right)}\right\|_{\infty}, j=0, \ldots, K$. Taking the above estimates into account, we find altogether

$$
B_{n} \leq(1+2 L C) \max \left\{C_{n}^{\left(\xi_{j}\right)} \mid j=0, \ldots, K\right\},
$$


where $L$ denotes the maximum of the constants $L^{\left(\xi_{k}, \xi_{\ell}\right)}, k, \ell \in\{0, \ldots, K\}$ associated in Lemma 2.3 to the domains $U_{\xi_{k}} \cap U_{\xi_{\ell}}$ and $\tilde{U}_{\xi_{k}} \cap \tilde{U}_{\xi_{\ell}}$ and $C$ denotes the constant associated to the cover $\left\{\tilde{U}_{\xi_{j}}\right\}_{j=0, \ldots, K}$ in Lemma 2.10.

By Lemma $4.5(1)$, the sequence $\left\{f_{n}\right\}_{n}$ is a $P$-asymptotic sequence, i.e. there exists $\hat{f} \in \mathcal{O}$ satisfying $J\left(f_{n}\right) \equiv \hat{f}$ for $n \in \mathbb{N}$ that is the $P$-asymptotic expansion of $f$ on $V \cap \Pi$. As we have seen this implies for every $\xi \in \mathbb{P}_{\mathbb{C}}^{1}$ that $\hat{f} \circ b_{\xi}$ is the $\left(P \circ b_{\xi}\right)$-asymptotic expansion of $f \circ b_{\xi}$ on any $\left(P \circ b_{\xi}\right)$-sector $\Pi_{P \circ b_{\xi}}(\alpha, \beta ; \mu)$ if $\mu$ is sufficiently small. The uniqueness of the $\left(P \circ b_{\xi}\right)$-asymptotic expansion (see Lemma $\left.4.5(2)\right)$ now implies that $\hat{f} \circ b_{\xi}=\hat{g}_{\xi}$ and the last assertion of the theorem is proved.

In the sequel, we want to improve Proposition 6.1 and also give a variant for $P$-asymptotic series. For this purpose, we need some additional notation and exploit the crucial observation that for any formal series $\hat{f} \in \hat{\mathcal{O}}$, the compositions $\hat{f} \circ b_{\xi}(\mathbf{v}), \xi \in \mathbb{P}_{\mathbb{C}}^{1}$, are not arbitrary formal series. Indeed, by the formulas of Subsection 2.2, we see that for each term in the series expansion of such a composition, the exponent of $v_{1}$ is smaller or equal to the one of $v_{2}$. Our charts were chosen so that we have this property for $\xi \in \mathbb{C}$ and for $\xi=\infty$. As a consequence, if we write $\hat{f} \circ b_{\xi}(\mathbf{v})$ as a series in powers of $v_{2}, \ldots, v_{d}$, the coefficients are polynomials of $v_{1}$, i.e.

$$
\hat{f} \circ b_{\xi}(\mathbf{v}) \in \mathbb{C}\left[v_{1}\right] \llbracket v_{2}, \ldots, v_{d} \rrbracket \quad \text { for } \hat{f} \in \hat{\mathcal{O}}, \xi \in \mathbb{P}_{\mathbb{C}}^{1} .
$$

We will work in a larger set than $\mathbb{C}\left[v_{1}\right] \llbracket v_{2}, \ldots, v_{d} \rrbracket$. We consider for positive $\rho$ the algebra $\mathcal{A}_{\rho}=\mathcal{O}_{b}(D(0, \rho)) \llbracket v_{2}, \ldots, v_{d} \rrbracket$ and regard it as a subset of $\hat{\mathcal{O}}$. It is endowed with the $\mathfrak{m}^{\prime}$-adic topology, where $\mathfrak{m}^{\prime}=$ $\left(v_{2}, \ldots, v_{d}\right) . \mathcal{A}_{\rho}$ is complete for this topology. It is finer than the one inherited from $\hat{\mathcal{O}}$ : Any sequence converging for the $\mathfrak{m}^{\prime}$-adic topology also converges for the $\mathfrak{m}$-adic topology. As $P \circ b_{\xi} \in \mathfrak{m}^{\prime}$ for any $\xi$, the $P \circ b_{\xi}$-adic topology generated by the powers of the ideal $\left(P \circ b_{\xi}(\mathbf{v})\right) \mathcal{A}_{\rho}$ is even finer than the $\mathfrak{m}^{\prime}$-adic topology. It is readily shown that $\mathcal{A}_{\rho}$ is also complete for the $P \circ b_{\xi}$-adic topology.

Lemma 6.2. Consider $\hat{g} \in \mathcal{A}_{\rho}$ for some positive $\rho$ and $\xi \in \mathbb{P}_{\mathbb{C}}^{1}$. Then there exists a $\left.\rho^{\prime} \in\right] 0, \rho[$ such that for all $\zeta \in \mathbb{C}$ sufficiently close to $\xi$, the composition $\hat{g} \circ\left(\phi_{\xi} \circ \phi_{\zeta}^{-1}\right)(\mathbf{v})$ is well defined and an element of $\mathcal{A}_{\rho^{\prime}}$.

Proof: In the case $\xi \in \mathbb{C}$ this follows immediately from the formula $\phi_{\xi} \circ \phi_{\zeta}^{-1}(\mathbf{v})=\left(v_{1}+\zeta-\xi, \mathbf{v}^{\prime}\right)$.

In the case $\xi=\infty$, we have $\phi_{\xi} \circ \phi_{\zeta}^{-1}(\mathbf{v})=\left(\left(\zeta+v_{1}\right)^{-1},\left(\zeta+v_{1}\right) v_{2}, \mathbf{v}^{\prime \prime}\right)$. If $f_{\boldsymbol{\beta}}\left(v_{1}\right), \boldsymbol{\beta} \in \mathbb{N}^{d-1}$, denotes the coefficient of $\hat{f}(\mathbf{v})$ in front of $\left(\mathbf{v}^{\prime}\right)^{\boldsymbol{\beta}}$ then the corresponding coefficient of $\hat{f} \circ\left(\phi_{\xi} \circ \phi_{\zeta}^{-1}\right)(\mathbf{v})$ is of the form $(\zeta+$ 
$\left.v_{1}\right)^{\beta_{2}} f_{\boldsymbol{\beta}}\left(\left(\zeta+v_{1}\right)^{-1}\right)$ which defines elements of $O_{b}\left(D\left(0, \rho^{\prime}\right)\right)$ for sufficiently small $\rho^{\prime}$ if $\zeta \in \mathbb{C}$ is sufficiently large.

Another consequence of (6.6) is used in Generalized Weierstrass Division (see Subsection 2.3). For a given injective linear form $\ell: \mathbb{N}^{d} \rightarrow \mathbb{R}_{+}$, the minimal exponent of a series $g$ is important in this context and determines the set $\Delta_{\ell}(g)$ used in the statements. It will be important for us to have an injective linear form such that the minimal exponent of $P \circ b_{\xi}(\mathbf{v})$ is the same for all but finitely many $\xi \in \mathbb{P}_{\mathbb{C}}^{1}$.

In the case $d=2$, we simply consider the leading terms of $P\left(x_{1}, x_{2}\right)$ with respect to the homogeneous valuation. Let $H\left(x_{1}, x_{2}\right)$ be their sum and $h>0$ their valuation. As $H \circ b_{\xi}\left(v_{1}, v_{2}\right)=H\left(1, \xi+v_{1}\right) v_{2}^{h}$ for $\xi \in \mathbb{C}$ and $H \circ b_{\xi}\left(v_{1}, v_{2}\right)=H\left(v_{1}, 1\right) v_{2}^{h}$ for $\xi=\infty$, the leading term is $v_{2}^{h}$ whatever the choice of $\ell$ provided $H(\xi) \neq 0$. (As we have identified $\xi \equiv[1, \xi]$ in the case $\xi \in \mathbb{C}$, resp. $\infty \equiv[0,1]$, we use here and in the sequel $H(\xi)=H(1, \xi)$, resp. $H(\infty)=H(0,1)$.) Observe that $\xi$ with $H(\xi)=0$ correspond to the tangent directions of the curve $P\left(x_{1}, x_{2}\right)=0$ in the origin. Geometrically, we have shown above that the germ $P$ is monomialized by blow-ups for all but some of these tangent directions. The tangent directions are also the intersection points of the exceptional divisor with the strict transform of $P\left(x_{1}, x_{2}\right)=0$ under blow up.

In the case $d>2$, consider any injective linear form $\mathcal{L}: \mathbb{N}^{d-2} \rightarrow \mathbb{R}_{+}$. Let $\mathcal{M}$ be the set of exponents $\boldsymbol{m}=\left(m_{3}, \ldots, m_{d}\right) \in \mathbb{N}^{d-2}$ such that there exists a nonvanishing term $\alpha x_{1}^{m_{1}} x_{2}^{m_{2}}\left(\boldsymbol{x}^{\prime \prime}\right)^{\boldsymbol{m}}$ in the series of $P(\boldsymbol{x})$ and denote by $\boldsymbol{a}$ the minimum of $\mathcal{M}$ with respect to the ordering $<_{\mathcal{L}}$ induced by $\mathcal{L}$. Now consider the sum $P_{\mathcal{L}}\left(x_{1}, x_{2}\right)$ of all terms in the series of $P(\boldsymbol{x})$ contain $\left(\boldsymbol{x}^{\prime \prime}\right)^{\boldsymbol{a}}$ and let $h \geq 0$ denote the homogenous valuation of $P_{\mathcal{L}}\left(x_{1}, x_{2}\right)$. Denote by $H_{\mathcal{L}}\left(x_{1}, x_{2}\right)$ the terms of $P_{\mathcal{L}}\left(x_{1}, x_{2}\right)$ of valuation $h$. In order to extend $\mathcal{L}$ to an injective linear form $\ell: \mathbb{N}^{d} \rightarrow \mathbb{R}_{+}$, choose a convenient $\ell_{2}$ such that

$$
\ell_{2} h<\min _{\boldsymbol{\beta} \in \mathcal{M} \backslash\{\boldsymbol{a}\}} \mathcal{L}(\boldsymbol{\beta})-\mathcal{L}(\boldsymbol{a})
$$

and with a convenient $\ell_{1}$ the linear form

$$
\ell(\boldsymbol{\beta})=\ell_{1} \beta_{1}+\ell_{2} \beta_{2}+\mathcal{L}\left(\boldsymbol{\beta}^{\prime \prime}\right) .
$$

Evaluating $H_{\mathcal{L}} \circ b_{\xi}\left(v_{1}, v_{2}\right)$ as above, it follows that $v_{2}^{h}\left(\mathbf{v}^{\prime \prime}\right)^{\boldsymbol{a}}$ is the minimal term of $P \circ b_{\xi}(\mathbf{v})$ for the ordering induced by $\ell$ provided $H_{\mathcal{L}}(\xi) \neq 0$. Observe that $H_{\mathcal{L}}$ can be a nonzero constant in the case $d>3$. In order to combine the cases $d=2$ and $d>2$, we put $\mathcal{L}=\emptyset$ in the former case and choose an arbitrary injective linear form $\ell$.

We summarize the discussion in the following lemma. 
Lemma 6.3. Consider an injective linear form $\mathcal{L}: \mathbb{N}^{d-2} \rightarrow \mathbb{R}_{+}$in the case $d>2$ and $\mathcal{L}=\emptyset$ in the case $d=2$. There exist an injective linear form $\ell: \mathbb{N}^{d} \rightarrow \mathbb{R}_{+}$extending $\mathcal{L}$ as in (6.7), a nonnegative integer $h$ and a homogeneous polynomial $H_{\mathcal{L}}\left(x_{1}, x_{2}\right)$ of degree $h$ such that for $\xi \in \mathbb{P}_{\mathbb{C}}^{1}$ the dominant term of $P \circ b_{\xi}(\mathbf{v})$ is $H_{\mathcal{L}}(\xi) v_{2}^{h}\left(\mathbf{v}^{\prime \prime}\right)^{a}$ provided $H_{\mathcal{L}}(\xi) \neq 0$.

Our next goal is to establish a statement analogous to Proposition 6.1 for $P$-asymptotic series. The first step is some kind of continuity for the notion of $P \circ b_{\xi}$-asymptotic sequences with respect to $\xi \in \mathbb{P}_{\mathbb{C}}^{1}$.

Lemma 6.4. Suppose that $\xi \in \mathbb{P}_{\mathbb{C}}^{1}$ and $\left\{g_{n}\right\}_{n \in \mathbb{N}}$ is a $P \circ b_{\xi}$-asymptotic sequence in $\mathcal{O}_{b}(D(\mathbf{0}, \rho))$ for some formal series $\hat{g} \in \hat{\mathcal{O}}$. Then there exists a $\left.\rho^{\prime} \in\right] 0, \rho\left[\right.$ such that $\hat{g} \in \mathcal{A}_{\rho^{\prime}}$ and hence there exists a neighborhood $\mathcal{V}$ of $\xi$ in $\mathbb{P}_{\mathbb{C}}^{1}$ such that for $\zeta \in \mathcal{V}, \hat{g}_{\zeta}(\mathbf{v})=\hat{g}\left(\phi_{\xi} \circ \phi_{\zeta}^{-1}(\mathbf{v})\right)$ defines a series in $\mathcal{A}_{\rho_{\zeta}}$ with some $\rho_{\zeta}>0$ and such that $\tilde{g}_{n}^{(\zeta)}(\mathbf{v})=g_{n}\left(\phi_{\xi} \circ \phi_{\zeta}^{-1}(\mathbf{v})\right)$ defines a $P \circ b_{\zeta}$-asymptotic sequence for $\hat{g}_{\zeta}$ in $\mathcal{O}_{b}\left(D\left(\mathbf{0} ; \rho_{\zeta}\right)\right)$.

Proof: By definition, we have $J\left(g_{n}\right) \equiv \hat{g} \bmod \left(P \circ b_{\xi}\right)^{n} \hat{\mathcal{O}}$ and thus $J\left(g_{n}-\right.$ $\left.g_{n+1}\right) \equiv 0 \bmod \left(P \circ b_{\xi}\right)^{n} \hat{\mathcal{O}}$ for all $n$. It is well known that this implies that $J\left(g_{n}-g_{n+1}\right) \equiv 0 \bmod \left(P \circ b_{\xi}\right)^{n} \mathcal{O}$ for all $n$. This is a very special case of Artin's approximation theorem. In the context of our work, it also follows using Lemma 2.4 for some arbitrary injective linear form $\ell$ in the cases $S=\mathbb{C} \llbracket \mathbf{v} \rrbracket$ and $S=\mathbb{C}\{\mathbf{v}\}$.

Here we apply Corollary 2.7 with the same $\ell$ for $P \circ b_{\xi}$ and obtain some $D_{s}, s$ small, such that the linear operators $Q, R: \mathcal{O}_{b}\left(D_{s}\right) \rightarrow \mathcal{O}_{b}\left(D_{s}\right)$ are continuous. Applying them several times, we obtain the existence of $q_{n} \in \mathcal{O}_{b}\left(D_{s}\right)$ such that $g_{n}-g_{n+1}=q_{n}\left(P \circ b_{\xi}\right)^{n}$ for all $n \in \mathbb{N}$. If $\rho^{\prime} \in$ ] $0, \rho$ is sufficiently small, this relation remains valid for the restrictions to $D\left(\mathbf{0}, \rho^{\prime}\right)$.

This means that $\left\{g_{n}\right\}_{n \in \mathbb{N}}$ is a Cauchy sequence in the $P \circ b_{\xi}$-adic topology of $\mathcal{A}_{\rho^{\prime}}$ and thus has a limit $\hat{h} \in \mathcal{A}_{\rho^{\prime}}$ in that topology, i.e.

$$
g_{n} \equiv \hat{h} \quad \bmod \left(P \circ b_{\xi}\right)^{n} \mathcal{A}_{\rho^{\prime}} \quad \text { for } n \in \mathbb{N} .
$$

Therefore also $J\left(g_{n}\right) \equiv \hat{h} \bmod \mathfrak{m}^{n}$ for all $n$. By the uniqueness of the limit we obtain that $\hat{g}=\hat{h}$ and hence the first statement.

If $\zeta \in \mathbb{P}_{\mathbb{C}}^{1}$ is sufficiently close to $\xi$ then right composition of every term in equations (6.8) with $\phi_{\xi} \circ \phi_{\zeta}^{-1}$ is well defined by Lemma 6.2. This implies that $\tilde{g}_{n}^{(\zeta)}$ tends to $\hat{g}_{\zeta}$ in the $P \circ b_{\zeta}$-adic topology of $\mathcal{A}_{\rho_{\zeta}}$ for some small $\rho_{\zeta}$ and hence the second statement.

Now we can prove the announced statement for $P$-asymptotic series analogous to Proposition 6.1. 
Proposition 6.5. Let $\hat{f} \in \hat{\mathcal{O}}$ be such that for all $\xi \in \mathbb{P}_{\mathbb{C}}^{1}$, the composition $\hat{f} \circ b_{\xi}$ is a $P \circ b_{\xi}$-asymptotic series. Then $\hat{f}$ is a P-asymptotic series.

Proof: By definition, for every $\xi \in \mathbb{P}_{\mathbb{C}}^{1}$ there exist $\rho_{\xi}>0$ and a sequence $\left\{g_{n}^{(\xi)}\right\}_{n}$ in $\mathcal{O}_{b}\left(D\left(\mathbf{0} ; \rho_{\xi}\right)\right)$ such that $g_{n}^{(\xi)} \equiv \hat{f} \circ b_{\xi} \bmod \left(P \circ b_{\xi}\right)^{n} \hat{\mathcal{O}}$. By the above lemma, $g_{n \zeta}^{(\xi)}:=g_{n}^{(\xi)} \circ\left(\phi_{\xi} \circ \phi_{\zeta}^{-1}\right)$ are well defined for $\zeta$ in some neighborhood $\mathcal{V}_{\xi}$ of $\xi$ in $\mathbb{P}_{\mathbb{C}}^{1}$ and they satisfy

$$
g_{n \zeta}^{(\xi)} \equiv \hat{f} \circ b_{\zeta} \quad \bmod \left(P \circ b_{\zeta}\right)^{n} \hat{\mathcal{O}} \quad \text { for } \zeta \in \mathcal{V}_{\xi} .
$$

Consider now the neighborhoods $U_{\xi}=\phi_{\xi}^{-1}\left(D\left(\mathbf{0} ; \rho_{\xi}\right)\right)$ of $(\xi, \mathbf{0})$ in the blow-up variety $M$. By reducing $\mathcal{V}_{\xi}$ or $\rho_{\xi}$, if necessary, we can assume that $\mathcal{V}_{\xi} \times\{\mathbf{0}\}=U_{\xi} \cap E$ (recall that $E \cong \mathbb{P}_{\mathbb{C}}^{1} \subseteq M$ is the exceptional divisor of the blow-up). Since the family $U_{\xi}, \xi \in \mathbb{P}_{\mathbb{C}}^{1}$, covers the exceptional divisor, a compact set, we can choose a finite subcover, say $U_{i}, i=$ $0, \ldots, K$, where $U_{i}$ corresponds to a certain $\xi_{i} \in \mathbb{P}_{\mathbb{C}}^{1}$. For the sake of brevity of notation, we put $g_{n}^{i}=g_{n}^{\left(\xi_{i}\right)}, \phi_{i}=\phi_{\xi_{i}}, \mathcal{V}_{i}=\mathcal{V}_{\xi_{i}}$, and $G_{n}^{i}=g_{n}^{i} \circ \phi_{i}$. Then (6.9) can be written

$$
G_{n}^{i} \circ \phi_{\zeta}^{-1} \equiv \hat{f} \circ b_{\zeta} \quad \bmod \left(P \circ b_{\zeta}\right)^{n} \hat{\mathcal{O}} \quad \text { for } \zeta \in \mathcal{V}_{i}, i=0, \ldots, K .
$$

This implies that

$$
\left(G_{n}^{i}-G_{n}^{j}\right) \circ \phi_{\zeta}^{-1} \equiv 0 \bmod \left(P \circ b_{\zeta}\right)^{n} \hat{\mathcal{O}}
$$

for all $n$ and $\zeta \in \mathcal{V}_{i} \cap \mathcal{V}_{j}, i, j \in\{0, \ldots, K\}$. Unfortunately this does not allow us to conclude that $G_{n}^{i}-G_{n}^{j}$ can be divided by $(P \circ b)^{n}$ on the intersections $U_{i} \cap U_{j}$ of their domains. These domains have to be reduced.

For every $\xi \in \mathbb{P}_{\mathbb{C}}^{1}$, we can choose an open neighborhood $D(\xi)$ of $(\xi, \mathbf{0})$ in $M$ with the following properties:

(1) For every $i \in\{0, \ldots, K\}$, if $\xi \in \mathcal{V}_{i}$ then $\operatorname{cl}(D(\xi)) \subset U_{i}$.

(2) The image $\phi_{\xi}\left(D_{\xi}\right)=D_{s(\xi)}$ is a neighborhood of 0 such that Lemma 2.6 is valid for $P \circ b_{\xi}$ and some arbitrarily chosen injective linear form $\ell$.

We obtain from (6.11) that for $i, j=0, \ldots, K$ and $\xi \in \mathcal{V}_{i} \cap \mathcal{V}_{j}$ there exists holomorphic functions $H_{n, \xi}^{i, j} \in \mathcal{O}_{b}(D(\xi)), n \in \mathbb{N}$, such that

$$
\left(G_{n}^{i}-G_{n}^{j}\right)(p)=H_{n, \xi}^{i, j}(p)(P \circ b(p))^{n} \quad \text { for } p \in D(\xi) .
$$

We just apply the operator $Q$ of Lemma 2.6 several times to $\left(G_{n}^{i}-G_{n}^{j}\right) \circ$ $\phi_{\xi}^{-1}$ and use (6.11).

On nonempty intersections $D(\xi) \cap D(\zeta)$, equations (6.12) imply that $H_{n, \xi}^{i, j}(p)=H_{n, \zeta}^{i, j}(p)$ for $p$ in the dense subset of all $p$ such that $(P \circ b)(p) \neq$ 
0. By continuity, we obtain that $H_{n, \xi}^{i, j}$ and $H_{n, \zeta}^{i, j}$ coincide on such an intersection $D(\xi) \cap D(\zeta)$. This allows us to define $U_{i}^{\prime}=\cup_{\xi \in \mathcal{V}_{i}} D(\xi)$ and holomorphic functions $H_{n}^{i, j}: U_{i}^{\prime} \cap U_{j}^{\prime} \rightarrow \mathbb{C}$ by

$$
H_{n}^{i, j}(p)=H_{n, \xi}^{i, j}(p) \quad \text { if } p \in U_{i}^{\prime} \cap U_{j}^{\prime}, p \in D(\xi) .
$$

By construction, the sets $U_{i}^{\prime}, i=0, \ldots, K$ are open, satisfy $U_{i}^{\prime} \cap E=$ $\mathcal{V}_{i} \times\{\mathbf{0}\}$ and $U_{i}^{\prime} \subset U_{i}$, and the restrictions of $G_{n}^{i}, n \in \mathbb{N}$, which we denote by the same name, satisfy

$$
G_{n}^{i}-G_{n}^{j}=H_{n}^{i, j}(P \circ b)^{n} \quad \text { for all } n \in \mathbb{N}, i, j \in\{0, \ldots, K\} .
$$

Now we can proceed as in the proof of Proposition 6.1.

For $i=0, \ldots, K$, we choose open sets $\tilde{U}_{i}$ such that $\operatorname{cl}\left(\tilde{U}_{i}\right) \subset U_{i}^{\prime}$ and the $\tilde{U}_{i}$ still form a cover of $E$. This is possible by the compactness of $E$. Then the functions $H_{n}^{j, k}$ are bounded on $\tilde{U}_{j} \cap \tilde{U}_{k}$ for all $n \in \mathbb{N}$ and all $j, k$. By Lemma 2.10 we find bounded holomorphic functions $L_{n}^{j}$, $j=0, \ldots, K$, on some open sets $\bar{U}_{j} \subset \tilde{U}_{j}$ forming an open cover of $E$ satisfying $H_{n}^{j, k}=L_{n}^{j}-L_{n}^{k}$ for all $n$ and all $j, k$. Then we define $F_{n}^{j}=$ $G_{n}^{j}-L_{n}^{j}(P \circ b)^{n}$ on $\bar{U}_{j}, j=0, \ldots, K$.

By construction, it follows that the functions $F_{n}^{j}, j=0, \ldots, K$, glue together and are hence restrictions of some holomorphic functions $F_{n}: \tilde{V} \rightarrow$ $\mathbb{C}$, where $\tilde{V}$ is some neighborhood of $E$. Hence they come from some holomorphic functions $f_{n}: V \rightarrow \mathbb{C}, V=b(\tilde{V})$, i.e. $F_{n}=f_{n} \circ b$. Observe that $\tilde{V}$ and $V$ (as the neighborhoods used before) are independent of $n$. By their construction and (6.10), it is easily verified that for all $\xi \in \mathbb{P}_{\mathbb{C}}^{1}$ and $n \in \mathbb{N}$, we have

$$
f_{n} \circ b_{\xi} \equiv \hat{f} \circ b_{\xi} \quad \bmod \left(P \circ b_{\xi}\right)^{n} \hat{\mathcal{O}} .
$$

Unfortunately it is not clear how to deduce directly that $f_{n} \equiv \hat{f} \bmod P^{n} \hat{\mathcal{O}}$ for all $n$.

It is more convenient to consider the sequence $\left\{f_{n}-f_{n+1}\right\}_{n \in \mathbb{N}}$. From (6.13) and Lemma 2.4 applied for $S=\hat{\mathcal{O}}$ and $S=\mathcal{O}$, we find that

$$
\left(f_{n}-f_{n+1}\right) \circ b_{\xi} \in\left(P \circ b_{\xi}\right)^{n} \mathcal{O}
$$

for all $n, \xi$. As in the proof of Lemma 2.2 this implies that $f_{n}-f_{n+1} \in$ $P^{n} \mathcal{O}$ for $n$. Hence $\left\{f_{n}\right\}_{n}$ is a Cauchy sequence for the $P$-adic topology and hence has a limit $\hat{g} \in \hat{\mathcal{O}}$ in it, i.e. $f_{n} \equiv \hat{g} \bmod P^{n} \hat{\mathcal{O}}$ for all $n$. By (6.13) and the uniqueness of the limit we obtain that $\hat{g} \circ b_{\xi}=\hat{f} \circ b_{\xi}$ for all $\xi \in \mathbb{P}_{\mathbb{C}}^{1}$. This implies that $\hat{g}=\hat{f}$ and completes the proof. 
Observe that if $\left|g_{n}^{(\xi)}\right| \leq K_{n}^{(\xi)}$ on $D\left(\mathbf{0} ; \rho_{\xi}\right)$, then $\left|H_{n}^{j, k}\right| \leq M^{j, k}\left(K_{n}^{\left(\xi_{j}\right)}+\right.$ $\left.K_{n}^{\left(\xi_{k}\right)}\right)$, where $M^{j, k}$ denotes the constant of Lemma 2.3 for $U_{j}^{\prime} \cap U_{k}^{\prime}$ and $\tilde{U}_{j} \cap \tilde{U}_{k}$. This implies with

$$
\left|L_{n}^{j}\right| \leq C \max \left\{\left|H_{n}^{j, k}\right| \mid j, k=0, \ldots, K\right\},
$$

where $C$ is the constant of Lemma 2.10 for the cover $\tilde{U}_{j}, j=0, \ldots, K$, that

(6.14) $\left|F_{n}^{j}\right| \leq K_{n}^{\left(\xi_{j}\right)}+\left(\sup _{\bar{U}_{j}}|P \circ b|\right)^{n} C \max \left\{M^{k, l}\left(K_{n}^{\left(\xi_{k}\right)}+K_{n}^{\left(\xi_{l}\right)}\right) \mid k, l=0, \ldots, K\right\}$.

This will be used later.

In order to improve Proposition 6.1, we first note a consequence of Lemma 6.4.

Lemma 6.6. Suppose that $g$ is holomorphic on $\Pi_{P \circ b_{\xi}}(a, b, R)$ and has some $P \circ b_{\xi}$-asymptotic expansion $\hat{g}_{\xi}$ on it. Then for $\zeta \in \mathbb{P}_{\mathbb{C}}^{1}$ close the $\xi$, the composition $\hat{g}_{\xi}\left(\phi_{\xi} \circ \phi_{\zeta}^{-1}(\mathbf{v})\right)$ is well defined and it is the $P \circ b_{\zeta^{-}}$asymptotic expansion of $g \circ\left(\phi_{\xi} \circ \phi_{\zeta}^{-1}\right)$ on $\Pi_{P \circ b_{\zeta}}\left(a, b, \rho_{\zeta}\right)$ for sufficiently small positive $\rho_{\zeta}$.

In particular, if $f$ holomorphic on $\Pi_{P}(a, b, R)$ and $\xi \in \mathbb{P}_{\mathbb{C}}^{1}$ such that $f \circ b_{\xi}=g$ satisfies the assumption, then the statement holds for $g \circ\left(\phi_{\xi} \circ\right.$ $\left.\phi_{\zeta}^{-1}\right)=f \circ b_{\zeta}$ and $\zeta$ close to $\xi$.

Proof: By definition, there exists $\rho>0$ and a $P \circ b_{\xi}$-asymptotic sequence $\left\{g_{n}\right\}_{n}$ for $\hat{g}_{\xi}$ on $\mathcal{O}_{b}(D(\mathbf{0} ; \rho))$ such that

$$
\left|f \circ b_{\xi}(\mathbf{v})-g_{n}(\mathbf{v})\right| \leq K_{n}\left|P \circ b_{\xi}(\mathbf{v})\right|^{n}
$$

for $\mathbf{v} \in \Pi_{P \circ b_{\xi}}(a, b, \rho)$. By the above lemma, $\tilde{g}_{n}^{(\zeta)}(\mathbf{v})=g_{n}\left(\phi_{\xi} \circ \phi_{\zeta}^{-1}(\mathbf{v})\right)$ defines a $P \circ b_{\zeta}$-asymptotic sequence for $\hat{g}_{\xi}\left(\phi_{\xi} \circ \phi_{\zeta}^{-1}(\mathbf{v})\right)$. Substitution of $\phi_{\xi} \circ \phi_{\zeta}^{-1}(\mathbf{v})$ for $\mathbf{v}$ in (6.15) yields that

$$
\left|f \circ b_{\zeta}(\mathbf{v})-\tilde{g}_{n}^{(\zeta)}(\mathbf{v})\right| \leq K_{n}\left|P \circ b_{\zeta}(\mathbf{v})\right|^{n}
$$

and the corollary is proved. Observe that for $\zeta$ close to $\xi$ and small $\mathbf{v}$ with $\left.\arg \left(P \circ b_{\zeta}(\mathbf{v})\right) \in\right] a, b\left[\right.$, we have $\phi_{\xi} \circ \phi_{\zeta}^{-1}(\mathbf{v}) \in \Pi_{P \circ b_{\xi}}(a, b, \rho)$. (Indeed, using the formulas for $\phi_{\xi} \circ \phi_{\zeta}^{-1}(\mathbf{v})$, we find that $\phi_{\xi} \circ \phi_{\zeta}^{-1}(\mathbf{0})$ is small if $\zeta$ is close to $\xi$. Hence by continuity, there is a $\left.\rho^{\prime} \in\right] 0, \rho[$ such that $\left|\phi_{\xi} \circ \phi_{\zeta}^{-1}(\mathbf{v})\right|<\rho$ if $|\mathbf{v}|<\rho^{\prime}$. The rest follows from $\left(P \circ b_{\xi}\right) \circ\left(\phi_{\xi} \circ \phi_{\zeta}^{-1}\right)=$ $\left.P \circ b_{\zeta}.\right)$

Next, we need a statement concerning the $\xi$-dependence of $T_{\ell}\left(f \circ b_{\xi}\right)$. 
Lemma 6.7. Consider $\mathcal{L}, \ell, h, H_{\mathcal{L}}$ as in Lemma 6.3 and let $\mathcal{Z}_{\mathcal{L}}$ denote the set of zeros of $H_{\mathcal{L}}$ in $\mathbb{P}_{\mathbb{C}}^{1}$. Let a bounded function $f \in \Pi_{P}(a, b, R)$ be given. Then for any $\mathcal{D}$ with $\operatorname{cl}(\mathcal{D})$ compact and contained in $\mathbb{C} \backslash \mathcal{Z}_{\mathcal{L}}$, there exist $\sigma, \rho>0$ and a function $G_{\mathcal{L}}: V(a, b, \sigma) \times \Omega \rightarrow \mathbb{C}, \Omega=\mathcal{D} \times$ $D(0 ; \rho)^{d-1}$, such that for $\xi \in \mathcal{D}$, the function $F_{\xi}(t, \mathbf{v})=T_{\ell}\left(f \circ b_{\xi}\right)(t, \mathbf{v})$ of Theorem 4.7 defined for $P \circ b_{\xi}$ satisfies $F_{\xi}(t, \mathbf{v})=G_{\mathcal{L}}\left(t,\left(v_{1}+\xi, \mathbf{v}^{\prime}\right)\right)$ for $t \in V(a, b, \sigma)$ and small $\mathbf{v}$.

This means essentially that the functions $T_{\ell}\left(f \circ b_{\xi}\right), \xi \in \mathbb{C} \backslash \mathcal{Z}_{\mathcal{L}}$ glue together to a single function except for shifts in the variable $v_{1}$.

Proof: Applying Theorem 4.7 for $\xi \in \mathbb{C} \backslash \mathcal{Z}_{\mathcal{L}}$ and with $P$ replaced by $P \circ b_{\xi}$ yields positive $\sigma_{\xi}, \rho_{\xi}$ and functions $F_{\xi}: V\left(a, b, \sigma_{\xi}\right) \times D\left(\mathbf{0} ; \rho_{\xi}\right) \rightarrow \mathbb{C}$ such that for any $t$, we have $J\left(F_{\xi}(t,).\right) \in \Delta_{\ell}\left(P \circ b_{\xi}\right)$ and $F_{\xi}\left(P \circ b_{\xi}(\mathbf{v}), \mathbf{v}\right)=$ $f\left(b_{\xi}(\mathbf{v})\right)$ for $\mathbf{v} \in \Pi_{P \circ b_{\xi}}\left(a, b, \rho_{\xi}\right)$. Observe that $F_{\xi}$ are uniquely determined by this property except for restrictions.

Putting $\tilde{F}_{\zeta}(t, \mathbf{v})=F\left(t, \phi_{\xi} \circ \phi_{\zeta}^{-1}(\mathbf{v})\right)=F_{\xi}\left(t,\left(v_{1}+\zeta-\xi, \mathbf{v}^{\prime}\right)\right)$ for $\zeta$ close to $\xi$ and sufficiently small $\mathbf{v}$, this function has the defining properties of $F_{\zeta}$. By the essential uniqueness of the latter, we obtain

$$
F_{\zeta}(t, \mathbf{v})=F_{\xi}\left(t, v_{1}+\zeta-\xi, \mathbf{v}^{\prime}\right)
$$

for $\zeta$ close to $\xi$ and sufficiently small $\mathbf{v}$. This means that $F_{\xi}$ and $F_{\zeta}$ are analytic continuations of each other, except for a shift in the $v_{1}$ component.

Consider now $\mathcal{D}$ compactly contained in $\mathbb{C} \backslash \mathcal{Z}_{\mathcal{L}}$ and choose a finite subset $A$ of the closure of $\mathcal{D}$ such that the disks $\xi+D\left(0, \rho_{\xi}\right)$ cover $\mathcal{D}$. This allows us to define

$$
G(t, \mathbf{v})=F_{\xi}\left(t, v_{1}-\xi, \mathbf{v}^{\prime}\right) \quad \text { for } t \in V(a, b, \sigma) \text { and } \mathbf{v} \in \Omega,
$$

if $v_{1} \in \xi+D\left(0, \rho_{\xi}\right)$ where $\sigma=\min \left\{\sigma_{\xi} \mid \xi \in A\right\}, \rho=\min \left\{\rho_{\xi} \mid \xi \in A\right\}$. Property (6.16) implies that the value of $G(t, \mathbf{v})$ is independent of the choice of $\xi$ with $v_{1} \in \xi+D\left(0, \rho_{\xi}\right)$. $G$ has the wanted properties by construction.

Now we are in a position to prove that the assumption on $f \circ b_{\xi}$ in Proposition 6.1 is only needed for finitely many $\xi \in \mathbb{P}_{\mathbb{C}}^{1}$.

Theorem 6.8. Consider $\mathcal{L}, \ell, h, H_{\mathcal{L}}, \mathcal{Z}_{\mathcal{L}}$ as in Lemma 6.7 and put $\mathcal{Z}=$ $\mathcal{Z}_{\mathcal{L}}$ if $H_{\mathcal{L}}$ is not a constant, $\mathcal{Z}=\{\xi\}$ with arbitrary $\xi \in \mathbb{P}_{\mathbb{C}}^{1}$ otherwise. If $f$ is holomorphic and bounded on $\Pi=\Pi_{P}(a, b, R)$ such that for $\xi \in$ $\mathcal{Z}$, the composition $f \circ b_{\xi}$ has some $P \circ b_{\xi}$-asymptotic expansion $\hat{g}_{\xi}$ on $\Pi_{P \circ b_{\xi}}(a, b, R)$ then $f$ has a P-asymptotic expansion $\hat{f}$ on $\Pi$ such that $\hat{f} \circ b_{\xi}=\hat{g}_{\xi}$ for $\xi \in \mathcal{Z}$. 
Proof: As we can always achieve this by a linear transformation in the $\left(x_{1}, x_{2}\right)$-space, we assume that $\infty \in \mathcal{Z}_{\mathcal{L}}$ if $H_{\mathcal{L}}$ is not constant. Indeed, if we replace $\left(x_{1}, x_{2}\right)$ by $\left(x_{1}, x_{2}\right) A$ with some invertible $2 \times 2$ matrix $A$, then $\mathcal{Z}_{\mathcal{L}}$ changes to $\mathcal{Z}_{\mathcal{L}} A^{-1}$, the set of all $[\alpha, \beta]$ such that $[\alpha, \beta] A \in \mathcal{Z}_{\mathcal{L}}$. The same transformation allows us to assume that $\mathcal{Z}=\{\infty\}$ in the case that $H_{\mathcal{L}}$ is constant.

This means that we have now $\infty \in \mathcal{Z}$ in all the cases. Put $\mathcal{Z}^{\prime}=$ $\mathcal{Z} \backslash\{\infty\}$. This set may be empty, in which case the proof is a little simplified. We only give the proof when $\mathcal{Z}^{\prime} \neq \emptyset$.

By Lemma $6.6, f \circ b_{\xi}$ has a $P \circ b_{\xi}$-asymptotic expansion for large $\xi \in \mathbb{C}$ and for $\xi$ close to $\mathcal{Z}^{\prime}$. To fix notation assume this is the case for $|\xi|>R / 2$ resp. $\operatorname{dist}\left(\xi, \mathcal{Z}^{\prime}\right)<2 r$.

Then we apply Lemma 6.7 with $\mathcal{D}=D(0, R) \backslash \cup_{\chi \in \mathcal{Z}^{\prime}} \operatorname{cl}(D(\chi, r))$ to $f$. We obtain $\sigma, \rho>0$ and a holomorphic function $G_{\mathcal{L}}: V(a, b, \sigma) \times \Omega \rightarrow \mathbb{C}$, $\Omega=\mathcal{D} \times D(0 ; \rho)^{d-1}$, such that for $\xi \in \mathcal{D}$, the function $T_{\ell}\left(f \circ b_{\xi}\right)(t, \mathbf{v})$ of Theorem 4.7 satisfies

$$
T_{\ell}\left(f \circ b_{\xi}\right)(t, \mathbf{v})=G_{\mathcal{L}}\left(t,\left(v_{1}+\xi, \mathbf{v}^{\prime}\right)\right) \quad \text { for } t \in V(a, b, \sigma) \text { and small } \mathbf{v} .
$$

By Theorem 4.9, $T_{\ell}\left(f \circ b_{\xi}\right)(t, \mathbf{v})$ has a uniform asymptotic expansion as $V\left(a, b, \sigma_{\xi}\right) \ni t \rightarrow 0$ if $\xi$ is in some neighborhood of $\partial \mathcal{D}$, the boundary of $\mathcal{D}$. Using (6.17) and the compactness of $\partial \mathcal{D}$, this implies that there exist some positive $\sigma, \rho$ such that $G(t, \mathbf{v})$ has a uniform limit as $V(a, b, \sigma) \ni t \rightarrow 0$ for $\mathbf{v}$ with $\left|\mathbf{v}^{\prime}\right|<\rho$, $\operatorname{dist}\left(v_{1}, \partial \mathcal{D}\right)<\rho$. By the Cauchy criterion, this is equivalent to

$$
\begin{aligned}
& \forall \varepsilon>0 \exists \delta>0 \forall t_{1}, t_{2} \in V(a, b, \sigma) \\
& \quad\left(\left|t_{1}\right|,\left|t_{2}\right|<\delta,\left|\mathbf{v}^{\prime}\right|<\rho, \operatorname{dist}\left(v_{1}, \partial \mathcal{D}\right)<\rho \Longrightarrow\left|G\left(t_{1}, \mathbf{v}\right)-G\left(t_{2}, \mathbf{v}\right)\right|<\varepsilon\right)
\end{aligned}
$$

Here we can apply the maximum modulus principle to $G(t, \mathbf{v})$ in the variable $v_{1}$ on the domain $\mathcal{D}$. This implies that

$$
\begin{aligned}
\forall \varepsilon>0 \exists \delta>0 \forall t_{1}, t_{2} \in V(a, b, \sigma) \\
\quad\left(\left|t_{1}\right|,\left|t_{2}\right|<\delta,\left|\mathbf{v}^{\prime}\right|<\rho, v_{1} \in \mathcal{D} \Longrightarrow\left|G\left(t_{1}, \mathbf{v}\right)-G\left(t_{2}, \mathbf{v}\right)\right|<\varepsilon\right)
\end{aligned}
$$

This means that $G(t, \mathbf{v})$ has some uniform limit as $V(a, b, \sigma) \ni t \rightarrow 0$, say $g_{0}(\mathbf{v})$, for $\mathbf{v} \in \Omega$.

In the same manner, we show that $\frac{1}{t}\left(G(t, \mathbf{v})-g_{0}(\mathbf{v})\right)$ has a uniform limit as $V(a, b, \sigma) \ni t \rightarrow 0$ for $\mathbf{v} \in \Omega$ etc. and obtain that $G(t, \mathbf{v})$ has an asymptotic expansion as $V(a, b, \sigma) \ni t \rightarrow 0$, uniformly for $\mathbf{v} \in \Omega$. By (6.17) and Theorem 4.9, this means that $f \circ b_{\xi}$ has a $P \circ b_{\xi}$-asymptotic expansion for every $\xi \in \mathcal{D}$. Now this is also the case for the remaining $\xi$ as discussed in the beginning of the proof. Proposition 6.1 implies the statement of the theorem. 
In the last part of this section, we want to improve Proposition 6.5 in a way similar to Theorem 6.8. We first show for any formal series $\hat{f} \in \hat{\mathcal{O}}$ that the coefficients series of all but finitely many of the series $T_{\ell}\left(\hat{f} \circ b_{\xi}\right)$, $\xi \in \mathbb{P}_{\mathbb{C}}^{1}$, can be combined into one formal series.

Lemma 6.9. Consider $\mathcal{L}, \ell, h, H_{\mathcal{L}}$ as in Lemma 6.3 and let $\mathcal{Z}_{\mathcal{L}}$ denote the set of zeros of $H_{\mathcal{L}}$ in $\mathbb{P}_{\mathbb{C}}^{1}$. Let $\mathcal{R}=\mathbb{C}\left[v_{1}, 1 / H_{\mathcal{L}}\left(1, v_{1}\right)\right]$ and $\mathcal{B}=$ $\mathcal{R} \llbracket v_{2}, \ldots, v_{d} \rrbracket$. Finally consider $\hat{f} \in \hat{\mathcal{O}}$. Then there exists a formal series $\hat{G}(t, \mathbf{v})=\sum_{n=0}^{\infty} \hat{g}_{n}(\mathbf{v}) t^{n} \in \mathcal{B} \llbracket t \rrbracket$ with the following property. For all $\xi \in \mathbb{C} \backslash \mathcal{Z}_{\mathcal{L}}$, the series

$$
T_{\ell}\left(\hat{f} \circ b_{\xi}\right)(t, \mathbf{v})=\sum_{n=0}^{\infty} \hat{f}_{n \xi}(\mathbf{v}) t^{n} \in \Delta_{\ell}\left(P \circ b_{\xi}\right) \llbracket t \rrbracket
$$

of Lemma 4.6 applied with $P \circ b_{\xi}$ in place of $P$ satisfies

$$
\hat{f}_{n \xi}(\mathbf{v})=J_{\xi}\left(\hat{g}_{n}\right)(\mathbf{v}) \quad \text { for } n \in \mathbb{N} .
$$

Here $J_{\xi}(h)(\mathbf{v})=\sum_{k=0}^{\infty} \frac{1}{k !} \frac{\partial^{k} h}{\partial\left(v_{1}\right)^{k}}\left(\xi, \mathbf{v}^{\prime}\right) v_{1}^{k}$ is obtained from some element $h \in \mathcal{B}$ by replacing each of its coefficients by its Taylor series in the point $\xi$. Since the coefficients are elements of $\mathcal{R}$ and hence rational functions of $v_{1}$ the denominator of which is a power of $H_{\mathcal{L}}\left(1, v_{1}\right)$, this is possible for $\xi \in \mathbb{C} \backslash \mathcal{Z}_{\mathcal{L}}$. Observe that $J_{\xi}$ is compatible with multiplication.

Proof: We consider the linear operator $B_{0}: \mathbb{C} \llbracket \boldsymbol{x} \rrbracket \rightarrow \mathbb{C}\left[v_{1}\right] \llbracket \mathbf{v}^{\prime} \rrbracket$ determined by $B_{0}\left(\boldsymbol{x}^{\boldsymbol{\alpha}}\right)=v_{1}^{\alpha_{2}} v_{2}^{\alpha_{1}+\alpha_{2}}\left(\mathbf{v}^{\prime \prime}\right)^{\boldsymbol{\alpha}^{\prime \prime}}$ and continuity with respect to $\mathfrak{m}$-adic topology of $\mathbb{C} \llbracket \boldsymbol{x} \rrbracket$ and the $\mathfrak{m}^{\prime}$-adic topology of $\mathbb{C}\left[v_{1}\right] \llbracket \mathbf{v}^{\prime} \rrbracket$.

Observe that $J_{\xi}\left(B_{0}(\hat{g})\right)=\hat{g} \circ b_{\xi} \in \mathbb{C} \llbracket \mathbf{v} \rrbracket$ for $\hat{g} \in \mathbb{C} \llbracket \boldsymbol{x} \rrbracket$. The difference of $B_{0}(\hat{g})$ and $\hat{g} \circ b_{0}$ is essentially that the former is in $\mathbb{C}\left[v_{1}\right] \llbracket \mathbf{v}^{\prime} \rrbracket$ whereas the latter is in $\mathbb{C} \llbracket \mathbf{v} \rrbracket$. Expansion of the polynomial coefficients in their Taylor series at the origin maps $B_{0}(\hat{g})$ to $\hat{g} \circ b_{0}$. The introduction of $B_{0}$ becomes more useful if $\mathbb{C}\left[v_{1}\right] \llbracket \mathbf{v}^{\prime} \rrbracket$ is considered as a subset of $\mathcal{B} ; \mathcal{B}$ cannot always be identified with a subset of $\mathbb{C} \llbracket \mathbf{v} \rrbracket$.

We consider $B_{0}(\hat{f})$ and $B_{0}(P)$ as elements of $\mathcal{B}=\mathcal{R} \llbracket v_{2}, \ldots, v_{d} \rrbracket$ and use the injective linear form $\ell^{\prime}\left(a_{2}, \ldots, a_{d}\right)=\ell_{2} a_{2}+\cdots+\ell_{d} a_{d}$ on $\mathbb{N}^{d-1}$. By Lemma 6.3 , the dominant term of $B_{0}(P)$ is $H_{\mathcal{L}}\left(1, v_{1}\right) v_{2}^{h}\left(\mathbf{v}^{\prime \prime}\right)^{\boldsymbol{a}}$ with $\boldsymbol{a}=\left(a_{3}, \ldots, a_{d}\right)$ and certain nonnegative $a_{j}$.

Now we apply Corollary 2.5 ; this is possible because $H_{\mathcal{L}}\left(1, v_{1}\right)$ is a unit of $\mathcal{R}$ by construction. Therefore we can write uniquely

$$
B_{0}(\hat{f})=\sum_{n=0}^{\infty} \hat{g}_{n} B_{0}(P)^{n}
$$


with certain $\hat{g}_{n} \in \mathcal{B} \cap \Delta_{\ell^{\prime}}\left(B_{0}(P)\right)$. These can be written

$$
\hat{g}_{n}(\mathbf{v})=\sum_{\boldsymbol{\beta} \in \mathbb{N}^{d-1}} g_{n, \boldsymbol{\beta}}\left(v_{1}\right)\left(\mathbf{v}^{\prime}\right)^{\boldsymbol{\beta}}
$$

with certain $g_{n, \boldsymbol{\beta}} \in \mathcal{R}$; by definition of $\Delta_{\ell^{\prime}}\left(B_{0}(P)\right)$ we have $g_{n, \boldsymbol{\beta}}=0$ if $\boldsymbol{\beta} \in(h, \boldsymbol{a})+\mathbb{N}^{d-1}$. We define

$$
\hat{G}(t, \mathbf{v})=\sum_{n=0}^{\infty} \hat{g}_{n}(\mathbf{v}) t^{n} \in \mathcal{B} \llbracket t \rrbracket
$$

and it remains to show that $\hat{G}$ has the wanted properties.

We can apply $J_{\xi}, \xi \in \mathbb{C} \backslash \mathcal{Z}_{\mathcal{L}}$, to equality (6.21) and obtain

$$
\hat{f} \circ b_{\xi}(\mathbf{v})=\sum_{n=0}^{\infty} J_{\xi}\left(\hat{g}_{n}\right)(\mathbf{v})\left(P \circ b_{\xi}(\mathbf{v})\right)^{n} a .
$$

Observe that $\hat{g}_{n} \in \Delta_{\ell^{\prime}}\left(B_{0}(P)\right)$ implies that for $\xi \in \mathbb{C} \backslash \mathcal{Z}_{\mathcal{L}}$, we have $J_{\xi}\left(\hat{g}_{n}\right) \in \Delta_{\ell}\left(P \circ b_{\xi}\right)$ because the leading terms used to define these vector spaces are $v_{2}^{h} v_{3}^{a_{3}} \cdots v_{d}^{a_{d}}$ respectively $v_{1}^{0} v_{2}^{h} v_{3}^{a_{3}} \cdots v_{d}^{a_{d}}$. This implies that (6.22) is actually the unique way to write $\hat{f} \circ b_{\xi}$ as a series $\hat{f} \circ b_{\xi}=$ $\sum_{n=0}^{\infty} \hat{f}_{n \xi}\left(P \circ b_{\xi}\right)^{n}$ with $\hat{f}_{n \xi} \in \Delta_{\ell}\left(P \circ b_{\xi}\right)$ if $\xi \in \mathbb{C} \backslash \mathcal{Z}_{\mathcal{L}}$. This means that $T_{\ell}\left(\hat{f} \circ b_{\xi}\right)(t, \mathbf{v})=\sum_{n=0}^{\infty} J_{\xi}\left(\hat{g}_{n}\right)(\mathbf{v}) t^{n}$ thus proving the lemma.

Now we can also improve Proposition 6.5.

Theorem 6.10. Consider $\mathcal{L}, \ell, h, H_{\mathcal{L}}, \mathcal{Z}_{\mathcal{L}}$, and $\mathcal{Z}$ as in Theorem 6.8. Let $\hat{f} \in \mathcal{O}$ be given such that $\hat{f} \circ b_{\xi}$ is a $P \circ b_{\xi}$-asymptotic series for $\xi \in \mathcal{Z}$. Then $\hat{f}$ is a $P$-asymptotic series.

Hence as in Theorem 6.8 , consideration of $\hat{f} \circ b_{\xi}$ for finitely many $\xi \in$ $\mathbb{P}_{\mathbb{C}}^{1}$ is already sufficient.

Proof: We can again assume that $\infty \in \mathcal{Z}$; otherwise we proceed as in the beginning of the proof of Theorem 6.8. By assumption and Lemma 6.2, $\hat{f} \circ b_{\xi}$ is a $P \circ b_{\xi}$-asymptotic series for large $\xi$ and for $\xi$ close to $\mathcal{Z}^{\prime}=$ $\mathcal{Z} \backslash\{\infty\}$. Therefore in the series expansions

$$
T_{\ell}\left(\hat{f} \circ b_{\xi}\right)=\sum_{n=0}^{\infty} \hat{f}_{n \xi}(\mathbf{v}) t^{n}
$$

of Corollary 4.10, the coefficients $\hat{f}_{n \xi}(\mathbf{v})$ are convergent series with a common radius of convergence, say $\rho_{\xi}>0$, for these $\xi$.

We can also apply the above Lemma 6.9 and obtain a series $\hat{G}(t, \mathbf{v})=$ $\sum_{n=0}^{\infty} \hat{g}_{n}(\mathbf{v}) t^{n} \in \mathcal{B} \llbracket t \rrbracket$ such that for $\xi \in \mathbb{C} \backslash \mathcal{Z}^{\prime}$ we have

$$
T_{\ell}\left(\hat{f} \circ b_{\xi}\right)=\sum_{n=0}^{\infty} J_{\xi}\left(\hat{g}_{n}\right)(\mathbf{v}) t^{n} \text {. }
$$


Here $J_{\xi}\left(\hat{g}_{n}\right)=\hat{f}_{n \xi}$ are convergent series for all $n$ and $\xi$ large or $\xi$ close to $\mathcal{Z}^{\prime}$. Therefore if we write

$$
J_{\xi}\left(\hat{g}_{n}\right)(\mathbf{v})=\sum_{\boldsymbol{\beta} \in \mathbb{N}^{d-1}} h_{n, \boldsymbol{\beta}}^{\xi}\left(v_{1}\right)\left(\mathbf{v}^{\prime}\right)^{\boldsymbol{\beta}}
$$

then there exist for these $\xi$ constants $K_{n, \xi}$ and $\rho_{\xi}>0$ such that

$$
\left|h_{n, \boldsymbol{\beta}}^{\xi}\left(v_{1}\right)\right| \leq K_{n, \xi} \rho_{\xi}^{-|\boldsymbol{\beta}|} \quad \text { for } \boldsymbol{\beta} \in \mathbb{N}^{d-1},\left|v_{1}\right|<\rho_{\xi} .
$$

For the coefficients $H_{n, \boldsymbol{\beta}}\left(v_{1}\right)$ in the expansion

$$
\hat{g}_{n}(\mathbf{v})=\sum_{\boldsymbol{\beta} \in \mathbb{N}^{d}-1} H_{n, \boldsymbol{\beta}}\left(v_{1}\right) \mathbf{v}^{\prime \boldsymbol{\beta}},
$$

which are elements of $\mathcal{R}=\mathbb{C}\left[v_{1}, 1 / H_{\mathcal{L}}\left(1, v_{1}\right)\right]$ and hence can be considered as holomorphic functions on $\mathbb{C} \backslash \mathcal{Z}^{\prime}$, this means that for $\xi$ large or $\xi$ close to $\mathcal{Z}^{\prime}$ there exist positive $\rho_{\xi}$ such that

$$
\left|H_{n, \boldsymbol{\beta}}\left(\xi+v_{1}\right)\right| \leq K_{n, \xi} \rho_{\xi}^{-|\boldsymbol{\beta}|} \quad \text { if }\left|v_{1}\right|<\rho_{\xi} .
$$

Consider now the domain $\mathcal{D}=D(0, R) \backslash \cup_{\chi \in \mathcal{Z}^{\prime}} \operatorname{cl}(D(\chi, r))$ where $r, R$ were chosen such that $(6.24)$ holds for $|\xi|>R / 2$ or $0<\operatorname{dist}\left(\xi, \mathcal{Z}^{\prime}\right)<2 r$. Then $H_{n, \boldsymbol{\beta}}$ is holomorphic on a neighborhood of the closure of $\mathcal{D}$ and, by compactness, there are $K_{n}, \rho>0$ such that for $v_{1}$ on the boundary of $\mathcal{D}$ we have $\left|H_{n, \boldsymbol{\beta}}\left(v_{1}\right)\right| \leq K_{n} \rho^{-|\boldsymbol{\beta}|}$. The maximum modulus principle implies here that

$$
\left|H_{n, \boldsymbol{\beta}}\left(v_{1}\right)\right| \leq K_{n} \rho^{-|\boldsymbol{\beta}|} \quad \text { for all } v_{1} \in \mathcal{D} .
$$

Using that $\hat{g}_{n}(\mathbf{v})=\sum_{\boldsymbol{\beta} \in \mathbb{N}^{d-1}} H_{n \boldsymbol{\beta}}\left(v_{1}\right)\left(\mathbf{v}^{\prime}\right)^{\boldsymbol{\beta}}$, we obtain that the coefficients of $t^{n}$ in (6.23) are convergent series on some common polydisk for all $\xi \in \mathcal{D}$. By Remark 4.11 (1) this proves that $\hat{f} \circ b_{\xi}$ is a $P \circ b_{\xi}$-asymptotic series for every $\xi \in \mathcal{D}$. As we already know this for the remaining $\xi \in \mathbb{P}_{\mathbb{C}}^{1}$, we can apply Proposition 6.5 and finally obtain the statement.

We end this section with a discussion of the compatibility of asymptotics with respect to an analytic germ and ramification. The proofs are much simpler here.

Proposition 6.11. Consider $P$ on $D(0 ; \rho), \Pi=\Pi_{P}(\alpha, \beta ; \rho)$, and $f: \Pi \rightarrow$ $\mathbb{C}$ holomorphic as above. Suppose that for some integer $k \geq 2$, the function $f \circ r_{k}$, restricted to $\Pi_{k}=\Pi_{P \circ r_{k}}(\alpha, \beta ; \tilde{\rho})$ with some sufficiently small $\tilde{\rho}$, has some formal series $\hat{g} \in \hat{\mathcal{O}}$ as $\left(P \circ r_{k}\right)$-asymptotic expansion on its domain.

Then there exists a formal series $\hat{f} \in \hat{\mathcal{O}}$ that is the P-asymptotic expansion of $f$ on $\Pi$ and it satisfies $\hat{f} \circ r_{k}=\hat{g}$. 
Proof: Using Definition 4.3, we can assume that there exists a sequence $\left\{g_{n}\right\}_{n}$ of bounded holomorphic functions $g_{n}: D(\mathbf{0} ; \tilde{\rho}) \rightarrow \mathbb{C}$ with $J\left(g_{n}\right) \rightarrow \hat{g}$ as $n \rightarrow \infty$ and constants $C_{n}$ such that

$$
\left|f \circ r_{k}(\mathbf{v})-g_{n}(\mathbf{v})\right| \leq C_{n}\left|P \circ r_{k}(\mathbf{v})\right|^{n}
$$

for $n \in \mathbb{N}$ and $\mathbf{v} \in \Pi_{k}$.

By construction, $f \circ r_{k}$ and $P \circ r_{k}$ are invariant under right composition with the rotation $R: \mathbf{v} \mapsto\left(e^{2 \pi i / k} v_{1}, \mathbf{v}^{\prime}\right)$ and as a consequence also $\Pi_{k}$. This implies using (6.25) that

$$
\left|f \circ r_{k}(\mathbf{v})-g_{n} \circ R^{j}(\mathbf{v})\right| \leq C_{n}\left|P \circ r_{k}(\mathbf{v})\right|^{n}
$$

for $n \in \mathbb{N}, j=0, \ldots, k-1$, and $\mathbf{v} \in \Pi_{k}$.

Consider now the sequence $\left\{h_{n}\right\}$ in $\mathcal{O}_{b}\left(\Pi_{k}\right)$ defined by $h_{n}=\frac{1}{k} \sum_{j=0}^{k-1} g_{n}$ 。 $R^{j}$. Clearly, we have

$$
\left|f \circ r_{k}(\mathbf{v})-h_{n}(\mathbf{v})\right| \leq C_{n}\left|P \circ r_{k}(\mathbf{v})^{n}\right| \quad \text { for } n \in \mathbb{N}, \mathbf{v} \in \Pi_{k}
$$

and $J\left(h_{n}\right) \rightarrow \hat{h}$ as $n \rightarrow \infty$ with $\hat{h}=\frac{1}{k} \sum_{j=0}^{k-1} \hat{g} \circ R^{j}$. Furthermore, $h_{n}$ and $\hat{h}$ are invariant under right composition with the rotation $R$. Hence there exist bounded holomorphic $f_{n}: \tilde{\Pi} \rightarrow \mathbb{C}, \tilde{\Pi}=\Pi_{P}(\alpha, \beta ; r)$ with some small positive $r$ and $\hat{f} \in \hat{\mathcal{O}}$ such that $f_{n} \circ r_{k}=h_{n}$ and $\hat{f} \circ r_{k}=\hat{h}$.

We obtain the wanted properties

$$
\left|f(\boldsymbol{x})-f_{n}(\boldsymbol{x})\right| \leq C_{n}\left|P(\boldsymbol{x})^{n}\right| \quad \text { for } n \in \mathbb{N}, \boldsymbol{x} \in \tilde{\Pi}
$$

and $J\left(f_{n}\right) \rightarrow \hat{f}$ as $n \rightarrow \infty$. This proves that $f$ has $\hat{f}$ as $P$-asymptotic expansion. The last assertion of the proposition follows from the fact that $f \circ r_{k}$ has $\hat{f} \circ r_{k}$ and $\hat{g}$ as $P \circ r_{k}$-asymptotic expansions.

We can also prove a statement for $P$-asymptotic series analogous to Proposition 6.11.

Proposition 6.12. Let $\hat{f} \in \hat{\mathcal{O}}$ and $k \in \mathbb{N}, k \geq 2$ be such that the composition $\hat{f} \circ r_{k}$ is a Por $_{k}$-asymptotic series. Then $\hat{f}$ is a P-asymptotic series.

Proof: Is it analogous to the previous one. If $\left\{g_{n}\right\}_{n}$ is a $P \circ r_{k}$-asymptotic sequence in $\Pi_{P \circ r_{k}}(\alpha, \beta ; \tilde{\rho})$ for some $\alpha, \beta$, $\tilde{\rho}$ with limit $\hat{f} \circ r_{k}$ then so is $\left\{h_{n}\right\}_{n}$ where $h_{n}=\frac{1}{k} \sum_{j=0}^{k-1} g_{n} \circ R^{j}, R$ the rotation $R: \mathbf{v} \mapsto\left(e^{2 \pi i / k} v_{1}, \mathbf{v}^{\prime}\right)$. Again the sequence $\left\{f_{n}\right\}_{n}$ in $\Pi_{P}(\alpha, \beta ; r), r>0$ sufficiently small, where $f_{n} \circ r_{k}$ is a restriction of $g_{n}$, is a $P$-asymptotic sequence for $\hat{f}$. 


\section{Gevrey asymptotic expansions and summability with respect to a germ}

7.1. Gevrey asymptotic expansions with respect to an analytic germ. We first give a definition analogous to Definition 4.3 and then a characterization analogous to Theorem 4.9. Theorem 4.7 is again crucial in order to establish a relation between asymptotics involving powers of the germ and single variable asymptotics with coefficients in a certain Banach space.

We consider again a nonzero germ of analytic function $P(\boldsymbol{x}) \in \mathcal{O}=$ $\mathbb{C}\left\{x_{1}, \ldots, x_{d}\right\}$, not a unit (i.e. $P(\mathbf{0})=0$ ), defined in $D(\mathbf{0} ; \rho), \rho>0$.

Definition 7.1. Given a $P$-sector $\Pi=\Pi_{P}(a, b ; \boldsymbol{r})$, and $f \in \mathcal{O}(\Pi)$, we will say that $\hat{f} \in \hat{\mathcal{O}}$ is the $P$-Gevrey asymptotic expansion of order $s$ of $f$, or more briefly its " $P$-s-(Gevrey) asymptotic expansion", if there exist $\rho>0$, a family $\left\{f_{n} \in \mathcal{O}_{b}(D(\mathbf{0} ; \rho))\right\}_{n}$, converging to $\hat{f}$ in the $\mathfrak{m}$-adic topology, and constants $K, A>0$ such that:

(1) $\forall n \in \mathbb{N}, \forall \boldsymbol{x} \in D(\mathbf{0} ; \rho),\left|f_{n}(\boldsymbol{x})\right| \leq K A^{n} \Gamma(s n+1)$.

(2) $\forall n \in \mathbb{N}, \forall \boldsymbol{x} \in \Pi \cap D(\mathbf{0} ; \rho),\left|f(\boldsymbol{x})-f_{n}(\boldsymbol{x})\right| \leq K A^{n} \Gamma(s n+1)|P(\boldsymbol{x})|^{n}$. A sequence $\left\{f_{n}\right\}_{n \in \mathbb{N}}$ satisfying (1) and $J\left(f_{n}\right) \equiv \hat{f} \bmod P^{n} \hat{\mathcal{O}}$ will be called a $P$-s-asymptotic sequence for $\hat{f}$.

Remark 7.2. (1) As for Definition 4.3, this definition is compatible with changes of variables and with multiplication of $P$ by a unit $U \in \mathcal{O}$. This is verified in the same way as in Remark 4.4.

(2) Again as for Definition 4.3, the definition is independent of the choice of the $P$-s-asymptotic sequence for $\hat{f}$. Indeed, if $\left\{f_{n}\right\}_{n \in \mathbb{N}}$ is as in the definition and if $\left\{g_{n}\right\}_{n \in \mathbb{N}}$ is another $P$-s-asymptotic sequence for $\hat{f}$, then $J\left(f_{n}\right) \equiv J\left(g_{n}\right) \bmod P^{n} \hat{\mathcal{O}}$ for all $n$. Here Lemma 2.6 can be applied in $\mathcal{O}_{b}\left(D_{s}\right), D_{s}=D\left(\mathbf{0} ;\left(s^{\ell_{1}}, \ldots, s^{\ell_{d}}\right)\right)$, for sufficiently small positive $s$ as it was done below Lemma 4.5. It shows that we can write $f_{n}-g_{n}=h_{n} P^{n}$, $h_{n}=Q^{n}\left(f_{n}-g_{n}\right) \in \mathcal{O}_{b}\left(D_{s}\right)$.

Hence, there exist some positive $\rho^{\prime}<\rho$ and positive constants $L, M$ such that

$$
\left|f_{n}(\boldsymbol{x})-g_{n}(\boldsymbol{x})\right| \leq L M^{n} \Gamma(s n+1)|P(\boldsymbol{x})|^{n}
$$

for all $\boldsymbol{x} \in D\left(\mathbf{0} ; \rho^{\prime}\right)$ and all $n$. This implies that property (2) also holds for $\left\{g_{n}\right\}_{n}$ with certain $K, A$.

(3) This definition agrees with the well-known definition of Gevrey asymptotics in one variable, i.e., if $P=x$. In fact, suppose that $\mid f(x)-$ $\left.f_{n}(x)\left|\leq K A^{n} \Gamma(n s+1)\right| x\right|^{n}$ on a sector $V$, with $f_{n} \in \mathcal{O}_{b}(D(0 ; \rho))$ satisfying $\left|f_{n}(x)\right| \leq K A^{n} \Gamma(n s+1)$. Write $f_{n}(x)=J_{n-1}\left(f_{n}\right)+\tilde{f}_{n}(x)$. Let $S$ 
again denote the operator defined by $S(f)=\frac{f(x)-f(0)}{x}$ on $\mathcal{O}_{b}(D(0 ; \rho))$. The maximum modulus principle implies that $\|S(f)\|_{\infty} \leq \frac{2}{\rho}\|f\|_{\infty}$. Moreover, $\tilde{f}_{n}(x)=x^{n} S^{n}\left(f_{n}\right)$. Hence

$$
\begin{aligned}
\left|f(x)-J_{n-1}(f)\right| & \leq\left|f(x)-f_{n}(x)\right|+\left|\tilde{f}_{n}(x)\right| \\
& \leq K A^{n} \Gamma(n s+1)|x|^{n}+|x|^{n}\left(\frac{2}{\rho}\right)^{n} K A^{n} \Gamma(n s+1),
\end{aligned}
$$

as wanted. The converse follows from the Gevrey property of the formal series $\hat{f}$ and is left to the reader.

As for general asymptotic expansions in a germ (see Theorem 4.9), we want to write Gevrey expansions in an expression in a standard form. Recall that this standard form depends on the choice of $\ell$ in the subsequent theorem.

Theorem 7.3. Let $\ell: \mathbb{N}^{d} \rightarrow \mathbb{R}_{+}$be an injective linear form, $P \in \mathcal{O} \backslash$ $\{0\}, P(\mathbf{0})=0$ and let $T_{\ell}$ be defined by Lemma 4.6 resp. Theorem 4.7. Let $\Pi$ be a P-sector, $f \in \mathcal{O}(\Pi), \hat{f} \in \hat{\mathcal{O}}$, and $s>0$. Then $f$ has $\hat{f}$ as $P$-s-asymptotic expansion on $\Pi$ if and only if there exist $\rho>0$ such that $T_{\ell} \hat{f}=\sum_{n=0}^{\infty} g_{n} t^{n} \in \mathcal{O}_{b}(D(\mathbf{0} ; \rho)) \llbracket t \rrbracket$ is a formal $s$-Gevrey series and one of the following two equivalent conditions holds:

(1) There exist constants $K$ and $A$ such that

$$
\left|f(\boldsymbol{x})-\sum_{n=0}^{N-1} g_{n}(\boldsymbol{x}) P(\boldsymbol{x})^{n}\right| \leq K A^{N} \Gamma(s N+1)|P(\boldsymbol{x})|^{N}
$$

for $\boldsymbol{x} \in \Pi \cap D(\mathbf{0} ; \rho), N \in \mathbb{N}$.

(2) The function $T_{\ell} f$ from Theorem 4.7 is defined on $V(a, b ; \sigma) \times$ $D(\mathbf{0}, \rho) \rightarrow \mathbb{C}$ for some positive $\sigma$ and satisfies

$$
T_{\ell} f \sim_{s} T_{\ell} \hat{f} \quad \text { as } V(a, b, \sigma) \ni t \rightarrow 0 .
$$

Remark 7.4. (1) In the case of $P(\boldsymbol{x})=\boldsymbol{x}^{\boldsymbol{\alpha}}$, statement (1) agrees with the second definition of monomial asymptotics of Gevrey type (see Definition/Proposition 3.11).

(2) As stated for general asymptotic expansions in a germ in Remark 4.11, products of functions having $P$-s-Gevrey asymptotic expansions also have $P$-s-asymptotic expansions. For a proof, consider $P$-s-asymptotic sequences $\left\{f_{n}\right\}_{n \in \mathbb{N}},\left\{g_{n}\right\}_{n \in \mathbb{N}}$ satisfying the inequalities in Definition 7.1 for $f, g$, respectively. Define

$$
h_{n}=\sum_{k=0}^{n} \sum_{j=0}^{k}\left(f_{j}-f_{j-1}\right)\left(g_{k-j}-g_{k-j-1}\right),
$$


(where $f_{-1}=g_{-1}=0$ ). A straightforward computation shows that $f(\boldsymbol{x}) g(\boldsymbol{x})-h_{n}(\boldsymbol{x})=\left(f(\boldsymbol{x})-f_{n}(\boldsymbol{x})\right) g(\boldsymbol{x})+\sum_{k=0}^{n}\left(f_{k}(\boldsymbol{x})-f_{k-1}(\boldsymbol{x})\right)\left(g(\boldsymbol{x})-g_{n-k}(\boldsymbol{x})\right)$.

Then it is first shown that $f_{n}(\boldsymbol{x})-f_{n-1}(\boldsymbol{x})$ and $g_{n}(\boldsymbol{x})-g_{n-1}(\boldsymbol{x})$ satisfy $s$-Gevrey estimates and then using the inequality

$$
\sum_{\nu=0}^{n} \Gamma((n-\nu) s+1) \Gamma(\nu s+1) \leq K_{s} \Gamma(n s+1)
$$

with some constant $K_{s}$ independent of $n, s$-Gevrey bounds for the modulus of this expression are obtained.

For a proof of (7.1), first use that the $\Gamma$-function is logarithmically convex and hence $\Gamma\left(x^{\prime}\right) \Gamma\left(y^{\prime}\right) \leq \Gamma(x) \Gamma(y)$ if $0<x<x^{\prime} \leq y^{\prime}<y$ with $x^{\prime}+y^{\prime}=x+y$. Therefore, if $N$ is the smallest integer $N \geq \frac{1}{s}$ and $n>2 N$ then the left hand side of (7.1) is smaller than $2 N \Gamma(n s+1)+(n-2 N+$ 1) $\Gamma(n s-N s+1) \Gamma(N s+1)$. Since $1<n s-N s+1 \leq n s$ and $n s>2$, we obtain the bound $2 N \Gamma(n s+1)+n \Gamma(n s) \Gamma(N s+1) \leq K_{s} \Gamma(n s+1)$ with $K_{s}=2 N+\frac{1}{s} \Gamma(N s+1)$ provided $n>2 N$. For the remaining finitely many cases it is sufficient to increase $K_{s}$ if necessary.

The compatibility of $P$-s-asymptotic expansions with partial derivatives could also be shown using Definition 7.1, but we prefer to prove it using our generalization Theorem 7.18 of the Ramis-Sibuya theorem.

(3) As is Remark 3.12, the series $T \hat{f}$ turns out to be $s$-Gevrey if we only suppose that it is the $s$-Gevrey asymptotic expansion of $T f$ in the sense of statement (2) of the theorem.

Proof of Theorem 7.3: Suppose $\left\{f_{n}\right\}_{n}$ is a $P$-s-asymptotic sequence for $\hat{f}$ satisfying the conditions of Definition 7.1 on a certain $P$-sector $\Pi$ and a certain polydisk $D(\mathbf{0}, \rho)$. As in the proof of Theorem 4.9, we choose a positive $\mu$ such that $D_{\mu} \subset D(\mathbf{0}, \rho)$ for the set $D_{\mu}$ of Lemma 2.6 and write (the restrictions to $D_{\mu}$ )

$$
f_{n}=\sum_{m=0}^{n-1} g_{m} P^{m}+Q^{n}\left(f_{n}\right) P^{n}
$$

where $g_{m}=R Q^{m}\left(f_{m+1}\right)=R Q^{m}\left(f_{\nu}\right)$ for all $\nu>m$. Then

$$
\begin{aligned}
\left|f_{n}(\boldsymbol{x})-\sum_{\nu=0}^{n-1} g_{\nu}(\boldsymbol{x}) P^{\nu}(\boldsymbol{x})\right| & =\left|Q^{n}\left(f_{n}\right)(\boldsymbol{x}) P(\boldsymbol{x})^{n}\right| \\
& \leq K\|Q\|^{n} A^{n} \Gamma(s n+1)|P(\boldsymbol{x})|^{n}
\end{aligned}
$$

for $n \in \mathbb{N}$ and $\boldsymbol{x} \in \Pi \cap D_{\mu}$. Together with condition (2) of Definition 7.1, this proves (1). 
Application of Theorem 4.7 with $K(t)=K A^{N} \Gamma(s N+1) t^{N}$ to the inequalities in (1) yields (2).

The proof of the converses is again trivial.

The same proof shows the following characterization of $P$-s-asymptotic sequences.

Definition/Proposition 7.5. Let $\ell: \mathbb{N}^{d} \rightarrow \mathbb{R}_{+}$be an injective linear form and let $\Delta_{\ell}(P)$ be defined by (2.4). Let $P \in \mathcal{O} \backslash\{\mathbf{0}\}, P(\mathbf{0})=0$, and $\hat{f} \in \hat{\mathcal{O}}$ be a formal series. Then the following statements are equivalent:

(1) There exists a P-s-asymptotic sequence for $\hat{f}$ in the sense of Definition 7.1.

(2) There exist $\rho>0$ and a sequence $\left\{g_{n}\right\}_{n}$ in $\mathcal{O}_{b}(D(\mathbf{0} ; \rho))^{\mathbb{N}}$ with $J\left(g_{n}\right) \in \Delta(P)$ for all $n$ such that $\hat{f}=\sum_{n=0}^{\infty} J\left(g_{n}\right) J(P)^{n}$ and $T_{\ell} \hat{f}=\sum_{n=0}^{\infty} g_{n} t^{n} \in \mathcal{O}_{b}(D(\mathbf{0} ; \rho)) \llbracket t \rrbracket$ is a formal $s$-Gevrey series.

If one and hence both statements are true, then $\hat{f}$ is called P-s-Gevrey.

As for general $P$-asymptotic expansions, $P$-s-asymptotic expansions are compatible with blow-ups.

Proposition 7.6. Consider $P \in \mathcal{O}(D(\mathbf{0} ; \rho)) \backslash\{0\}, P(\mathbf{0})=0, \Pi_{P}=$ $\Pi_{P}(a, b ; r)$, and $f \in \mathcal{O}\left(\Pi_{P}\right)$. Then, $f$ has a $P$-s-Gevrey asymptotic expansion on $\Pi_{P}$ if and only if for every $\xi \in \mathbb{P}_{\mathbb{C}}^{1}$, there exists a positive $r_{\xi}$ such that $f \circ b_{\xi}$ has a $P \circ b_{\xi}-s$-Gevrey asymptotic expansion on $\Pi_{P \circ b_{\xi}}\left(a, b ; r_{\xi}\right)$.

Proof: We follow the proof of Theorem 6.1, taking $C_{n}^{(\xi)}=C_{\xi} A_{\xi}^{n} \Gamma(n s+1)$. By the compactness of $\mathbb{P}_{\mathbb{C}}^{1}$, it suffices to consider only a finite number of points in $\mathbb{P}_{\mathbb{C}}^{1}$, say $\xi_{0}, \xi_{1}, \ldots, \xi_{K}$, so we can omit the dependence on $\xi$ of the above constants, taking the maximum of their values. Using the notation of Theorem 6.1 , there exists a constant $L$ such that

$$
\left\|H_{n}^{\left(\xi_{i}, \xi_{j}\right)}\right\| \leq L 2 C A^{n} \Gamma(n s+1),
$$

and $\tilde{C}$ such that

$$
\left\|L_{n}^{\left(\xi_{j}\right)}\right\| \leq \tilde{C} L 2 C A^{n} \Gamma(n s+1) .
$$

Similarly, the constants in (6.4) and (6.5) are of $s$-Gevrey type, i.e. the construction in the proof of Theorem 6.1 yields a $P$ - $s$-Gevrey asymptotic expansion if the given expansions of $f \circ b_{\xi}$ are $P \circ b_{\xi^{-}}-$-Gevrey asymptotic expansions for every $\xi$ in $\mathbb{P}_{\mathbb{C}}^{1}$.

As for ordinary $P$-asymptotic expansions, the above proposition can be improved. Its assumption is in fact only necessary for a finite number of $\xi$ in $\mathbb{P}_{\mathbb{C}}^{1}$. 
Theorem 7.7. Consider $\mathcal{L}, \ell, h, H_{\mathcal{L}}, \mathcal{Z}_{\mathcal{L}}$ as in Lemma 6.7 and put $\mathcal{Z}=$ $\mathcal{Z}_{\mathcal{L}}$ if $H_{\mathcal{L}}$ is not a constant, $\mathcal{Z}=\{\xi\}$ with arbitrary $\xi \in \mathbb{P}_{\mathbb{C}}^{1}$ otherwise. Let $s>0$. If $f$ is holomorphic and bounded on $\Pi=\Pi_{P}(a, b, R)$ such that for $\xi \in \mathcal{Z}$, the composition $f \circ b_{\xi}$ has some $P \circ b_{\xi}$-s-asymptotic expansion $\hat{g}_{\xi}$ on $\Pi_{P \circ b_{\xi}}(a, b, R)$ then $f$ has a P-s-asymptotic expansion $\hat{f}$ on $\Pi$ such that $\hat{f} \circ b_{\xi}=\hat{g}_{\xi}$ for $\xi \in \mathcal{Z}$.

Proof: We follow the proof of Theorem 6.8 and essentially add Gevrey estimates. We again can assume that $\infty \in \mathcal{Z}$. We first carry Lemma 6.6 over to $P$-s-Gevrey asymptotics. It suffices to use $K_{n}=C A^{n} \Gamma(s n+1)$ in its proof. As we will need this statement again, we write it down as a lemma.

Lemma 7.8. Suppose that $g$ is holomorphic on $\Pi_{P \circ b_{\xi}}(a, b, R)$ and has some $P \circ b_{\xi}-s$-Gevrey asymptotic expansion $\hat{g}_{\xi}$ on it. Then for $\zeta \in \mathbb{P}_{\mathbb{C}}^{1}$ close the $\xi$, the composition $\hat{g}_{\xi}\left(\phi_{\xi} \circ \phi_{\zeta}^{-1}(\mathbf{v})\right)$ is well defined and it is the $P \circ b_{\zeta}-s$-Gevrey asymptotic expansion of $g \circ\left(\phi_{\xi} \circ \phi_{\zeta}^{-1}\right)$ on $\Pi_{P \circ b_{\zeta}}\left(a, b, \rho_{\zeta}\right)$ for sufficiently small positive $\rho_{\zeta}$.

In particular, if $f$ holomorphic on $\Pi_{P}(a, b, R)$ and $\xi \in \mathbb{P}_{\mathbb{C}}^{1}$ such that $f \circ b_{\xi}=g$ satisfies the assumption, then the statement holds for $g \circ\left(\phi_{\xi} \circ\right.$ $\left.\phi_{\zeta}^{-1}\right)=f \circ b_{\zeta}$ and $\zeta$ close to $\xi$.

We obtain that $f \circ b_{\xi}$ has a $P$-s-Gevrey asymptotic expansion for $\xi$ large or $\xi$ close to $\mathcal{Z}^{\prime}=\mathcal{Z} \backslash\{\infty\}$.

In a second step, we use again Lemma 6.7 on some domain $\mathcal{D}=$ $D(0, R) \backslash \cup_{\chi \in \mathcal{Z}^{\prime}} \operatorname{cl}(D(\chi, r))$. It yields $\sigma, \rho>0$ and a holomorphic function $G_{\mathcal{L}}: V(a, b, \sigma) \times \Omega \rightarrow \mathbb{C}, \Omega=\mathcal{D} \times D(0 ; \rho)^{d-1}$, such that for $\xi \in \mathcal{D}$, the function $T_{\ell}\left(f \circ b_{\xi}\right)(t, \mathbf{v})$ of Theorem 4.7. satisfies

$$
T_{\ell}\left(f \circ b_{\xi}\right)(t, \mathbf{v})=G_{\mathcal{L}}\left(t,\left(v_{1}+\xi, \mathbf{v}^{\prime}\right)\right) \quad \text { for } t \in V(a, b, \sigma) \text { and small } \mathbf{v} \text {. }
$$

It had been shown in the proof of Theorem 4.7 that $G_{\mathcal{L}}(t, \mathbf{v})$ has a uniform asymptotic expansion on $\Omega$ as $V(a, b, \rho) \ni t \rightarrow 0$. Denote it by $G_{\mathcal{L}}(t, \mathbf{v}) \sim \sum_{n=0}^{\infty} g_{n}(\mathbf{v}) t^{n}$. As $T_{\ell}\left(f \circ b_{\xi}\right)(t, \mathbf{v})$ has a Gevrey asymptotic expansion by assumption and Theorem 7.3, provided $\xi$ is close to the boundary of $\mathcal{D}$, we obtain using (7.2) and the compactness of $\partial \mathcal{D}$ again that there are positive constants $K, A$ such that

$$
\left|G_{\mathcal{L}}(t, \mathbf{v})-\sum_{n=0}^{N-1} g_{n}(\mathbf{v}) t^{n}\right| \leq K A^{N} \Gamma(s N+1)|t|^{N}
$$

for all $n \in \mathbb{N}$, all $t \in V(a, b, \rho)$, and all $\mathbf{v}$ such that $\left|\mathbf{v}^{\prime}\right|<\rho$ and $\operatorname{dist}\left(v_{1}, \partial \mathcal{D}\right)<\rho$. Here we use again the maximum modulus principle in the variable $v_{1}$ on the domain $\mathcal{D}$ and obtain that (7.3) is valid for all the above $n, t$ and all $\mathbf{v} \in \Omega$. This shows using (7.2) and Theorem 7.3 
again that $f \circ b_{\xi}$ has a $P \circ b_{\xi^{-}}$-Gevrey asymptotic expansion for all $\xi \in \mathcal{D}$. As this is known for the remaining $\xi$ already, we have it for all $\xi \in \mathbb{P}_{\mathbb{C}}^{1}$. Proposition 7.6 allows us to conclude.

We can also carry over the statements of Section 6 concerning $P$-asymptotic series to $P$-s-Gevrey asymptotic series. We first do so for Proposition 6.5.

Proposition 7.9. Let $\hat{f} \in \hat{\mathcal{O}}$ be such that for all $\xi \in \mathbb{P}_{\mathbb{C}}^{1}$, the composition $\hat{f} \circ b_{\xi}$ is a $P \circ b_{\xi}-s$-Gevrey asymptotic series. Then $\hat{f}$ is a $P$-s-Gevrey asymptotic series.

Proof: The proof of Proposition 6.5 carries over unchanged. Just observe that we have here special constants $K_{n}^{(\xi)}=C_{\xi} A_{\xi}^{n} \Gamma(s n+1)$ with $C_{\xi}, A_{\xi}$ independent of $n$ and that by (6.14), we obtain such Gevrey estimates also for the $F_{n}^{j}$ and hence for the functions $f_{n}$.

As for Proposition 6.5, the above proposition can be improved insofar as it is sufficient to assume the Gevrey character of $\hat{f} \circ b_{\xi}$ for finitely many $\xi$ only.

Theorem 7.10. Consider $\mathcal{L}, \ell, h, H_{\mathcal{L}}, \mathcal{Z}_{\mathcal{L}}$, and $\mathcal{Z}$ as in Theorem 7.7. Let $\hat{f} \in \mathcal{O}$ be given such that $\hat{f} \circ b_{\xi}$ is a $P \circ b_{\xi}-s$-Gevrey asymptotic series for $\xi \in \mathcal{Z}$. Then $\hat{f}$ is a $P$-s-Gevrey asymptotic series.

Proof: In the proof of Theorem 6.10, only the constants have to be modified: We have $K_{n, \xi}=C_{\xi} A_{\xi}^{n} \Gamma(s n+1)$ with some positive $C \xi, A_{\xi}$ independent of $n$ and as a consequence later $K_{n}=C A^{n} \Gamma(s n+1)$ with certain positive $C, A$. Details are left to the readers.

Remark 7.11. Consider the formal series $\hat{f}\left(x_{1}, x_{2}\right)=\sum_{n=0}^{\infty} n ! x_{2}^{2 n}\left(x_{1} x_{2}\right)^{n}$ and the monomial $P\left(x_{1}, x_{2}\right)=x_{1} x_{2}$ as a special germ of an analytic function. Whatever $\ell$, we have $\left(T_{\ell} \hat{f}\right)(t)\left(x_{1}, x_{2}\right)=\sum_{n=0}^{\infty} n ! x_{2}^{2 n} t^{n}$ and therefore $\hat{f}$ is a $P$-1-Gevrey asymptotic series.

Whatever $\ell$, we have $\mathcal{Z}_{\ell}=\{0, \infty\}$. We calculate

$$
\hat{f} \circ b_{0}\left(v_{1}, v_{2}\right)=\hat{f}\left(v_{2}, v_{1} v_{2}\right)=\sum_{n=0}^{\infty} n ! v_{1}^{3 n} v_{2}^{4 n}=\sum_{n=0}^{\infty} n ! v_{1}^{n}\left(v_{1} v_{2}^{2}\right)^{2 n}
$$

and $P \circ b_{0}\left(v_{1}, v_{2}\right)=v_{1} v_{2}^{2}$. Hence $\hat{f} \circ b_{0}$ is a $P \circ b_{0}-\frac{1}{2}$-Gevrey series. We also calculate

$$
\hat{f} \circ b_{\infty}\left(v_{1}, v_{2}\right)=\hat{f}\left(v_{1} v_{2}, v_{2}\right)=\sum_{n=0}^{\infty} n ! v_{1}^{n} v_{2}^{4 n}=\sum_{n=0}^{\infty} n ! v_{2}^{2 n}\left(v_{1} v_{2}^{2}\right)^{n}
$$

and again $P \circ b_{\infty}\left(v_{1}, v_{2}\right)=v_{1} v_{2}^{2}$. Hence $\hat{f} \circ b_{\infty}$ is $P \circ b_{\infty}-1$-Gevrey. 
Using Theorem 7.10, this confirms that $\hat{f}$ is a $P$-1-Gevrey asymptotic series. It also shows that we need to consider the blow-ups $\hat{f} \circ b_{\xi}$ for all $\xi \in \mathcal{Z}_{\ell}$ in order to conclude: Theorem 7.10 seems to be sharp with respect to the number of points to be considered in the exceptional divisor.

$P$-s-Gevrey asymptotic expansions and series are also compatible with ramification.

Proposition 7.12. Consider $P$ on $D(0 ; \rho), \Pi=\Pi_{P}(\alpha, \beta ; \rho)$, and $f: \Pi \rightarrow$ $\mathbb{C}$ holomorphic. Suppose that for some integer $k \geq 2$ and positive $s$, the function $f \circ r_{k}$, restricted to $\Pi_{k}=\Pi_{P \circ r_{k}}(\alpha, \beta ; \tilde{\rho})$ with some sufficiently small $\tilde{\rho}$, has some formal series $\hat{g} \in \hat{\mathcal{O}}$ as $\left(P \circ r_{k}\right)-s$-Gevrey asymptotic expansion on its domain.

Then there exists a formal series $\hat{f} \in \hat{\mathcal{O}}$ that is the P-s-Gevrey asymptotic expansion of $f$ on $\Pi$ and it satisfies $\hat{f} \circ r_{k}=\hat{g}$.

Proposition 7.13. Let $\hat{f} \in \hat{\mathcal{O}}, k \in \mathbb{N}, k \geq 2$, and $s>0$ be such that the composition $\hat{f} \circ r_{k}$ is a $P \circ r_{k}-s$-Gevrey asymptotic series. Then $\hat{f}$ is a $P$-s-Gevrey asymptotic series.

The proofs are analogous to the ones of Propositions 6.11 and 6.12. One just has to add Gevrey estimates.

As for monomial Gevrey asymptotics, functions having a $P$-s-asymptotic expansion with vanishing series are exponentially small.

Lemma 7.14. If $\Pi$ is a $P$-sector for a certain $P$ and if $f \in \mathcal{O}$ ( $)$ has a $P$-s-Gevrey asymptotic expansion where $\hat{f}=0$, then for all sufficiently small $R^{\prime}>0$ there exist $C, B>0$ such that on $\tilde{\Pi}$

$$
|f(\boldsymbol{x})| \leq C \exp \left(-\frac{B}{|P(\boldsymbol{x})|^{1 / s}}\right) \quad \text { for } \boldsymbol{x} \in \Pi,|\boldsymbol{x}|<R^{\prime} .
$$

Proof: As $\hat{f}=0$ implies that all $g_{n}$ of Theorem 7.3 vanish, we have that $|f(\boldsymbol{x})| \leq K A^{N} \Gamma(s N+1)|P(\boldsymbol{x})|^{N}$ for all sufficiently small $\boldsymbol{x} \in \Pi$ and for all $N \in \mathbb{N}$. Again we choose $N$ close to the optimal value $(A|P(\boldsymbol{x})|)^{-1 / s}$ and Stirling's formula yields the statement.

As a consequence of Definition/Proposition 7.5 we can also construct a function that have a prescribed $P$-s-Gevrey series as its $P$-s-asymptotic expansion.

Proposition 7.15 (Borel-Ritt-Gevrey). Let $P \in \mathcal{O} \backslash\{0\}, P(\mathbf{0})=0$ as before, $\hat{f}$ a P-s-Gevrey series, and $\Pi=\Pi_{P}(a, b ; r)$ a P-sector of opening $b-a<s \pi$. Then there exist $\rho>0$ and $f \in \mathcal{O}(\Pi \cap D(\mathbf{0} ; \rho))$ such that $f$ has $\hat{f}$ as $P$-s-Gevrey asymptotic expansion on $\Pi \cap D(\mathbf{0} ; \rho)$. 
Proof: Statement (2) of Definition/Proposition 7.5 yields the existence of $\rho>0$ and of a sequence $\left\{g_{n}\right\}_{n} \in \mathcal{O}_{b}(D(\mathbf{0} ; \rho))^{\mathbb{N}}$ with $J\left(g_{n}\right) \in \Delta(P)$ for all $n$ such that $\hat{f}=\sum_{n=0}^{\infty} J\left(g_{n}\right) J(P)^{n}$ and $\sum_{n=0}^{\infty} g_{n} T^{n} \in \mathcal{O}_{b}(D(\mathbf{0} ; \rho)) \llbracket T \rrbracket$ is a formal $s$-Gevrey series. Now let $V=V(a, b ; \mu)$, where $\mu>|P(\boldsymbol{x})|$ for all $\boldsymbol{x} \in D(\mathbf{0} ; \rho)$. By the Borel-Ritt-Gevrey theorem in Banach spaces (see Theorem 3.1), there exists $F \in \mathcal{O}(D(\mathbf{0} ; r) \times V)$ having $\sum_{m} g_{m} T^{m}$ as $s$-Gevrey asymptotic expansion at $T=0$. The function $f$ defined by $f(\boldsymbol{x})=F(\boldsymbol{x}, P(\boldsymbol{x}))$ gives the result.

As in the classical and monomial asymptotics, functions having Gevrey asymptotic expansions in a germ can be characterized by completing them to a family almost covering a neighborhood of $\mathbf{0}$ such that the differences of any two of them is exponentially small if the intersection of their domain is nonempty. In this context, covers of polydisks $D(\mathbf{0} ; R)$ outside the zero set of $P$ will be important.

Definition 7.16. A $P$-cover denotes a family $\left\{\Pi_{i}\right\}_{i \in I}, I$ some finite set, of $P$-sectors that covers the open set $D(\mathbf{0} ; R) \backslash\{P(\boldsymbol{x})=0\}$ for some $R>0$. Given such a $P$-cover $\mathcal{P}=\left\{\Pi_{i}\right\}_{i \in I}$, a $P$ - $k$-quasifunction on $\mathcal{P}$ is a family $\left(f_{i}\right)_{i \in I}$ of bounded holomorphic functions $f_{i} \in \mathcal{O}_{b}\left(\Pi_{i}\right)$, such that whenever $\Pi_{i} \cap \Pi_{j} \neq \emptyset$ there exist constants $C$ and $B$ satisfying

$$
\left|f_{i}(\boldsymbol{x})-f_{j}(\boldsymbol{x})\right| \leq C \exp \left(-\frac{B}{|P(\boldsymbol{x})|^{k}}\right) \quad \text { for all } \boldsymbol{x} \in \Pi_{i} \cap \Pi_{j} .
$$

Proposition 7.15 and Lemma 7.14 imply that a function having an $s$ Gevrey asymptotic expansion in a germ can be completed to a $P$ - $k$-quasifunction, $k=1 / s$.

Proposition 7.17. Consider a holomorphic function $f \in \mathcal{O}(\Pi)$ having a P-s-Gevrey asymptotic expansion on $\Pi$. Then there exist $\rho>0$, a $P$-cover $\Pi_{1}, \Pi_{2}, \ldots, \Pi_{r}$ of $D(\mathbf{0}, \rho) \backslash\{P=0\}$ with $\Pi_{1}=D(\mathbf{0}, \rho) \cap \Pi$, and a P-k-quasifunction $\left(f_{1}, f_{2}, \ldots, f_{r}\right)$ on it such that $k=1 / s$ and $f_{1}=\left.f\right|_{\Pi_{1}}$.

Proof: We need to assume that the opening of each $\Pi_{i}, i>1$, is not greater than $s \pi$. Then there exist $f_{i} \in \mathcal{O}\left(\Pi_{i}\right)(i>1)$ having the same $P$-s-asymptotic expansion as $f$; this is possible thanks to Proposition 7.15 provided $\Pi_{i}$ are contained in a sufficiently small polydisk. If $\Pi_{i} \cap \Pi_{j} \neq \emptyset$ then $f_{i}-f_{j}$ have a $P$-s-asymptotic expansion on it and $\left(f_{i}-f_{j}\right)(\boldsymbol{x}) \sim 0$. Lemma 7.14 now implies that the $f_{i}$ can be combined to a $P$-k-quasifunction, $k=1 / s$.

As for classical and monomial asymptotics, the converse is also true. This result, which generalizes the classical Ramis-Sibuya theorem (Theorem 3.4) and the version for monomial asymptotics (Theorem 3.14), 
are the most important means to establish the existence of $P$-s-Gevrey asymptotic expansions.

Theorem 7.18. Suppose that the P-sectors $\Pi_{j}=\Pi_{P}\left(a_{j}, b_{j} ; r\right), 1 \leq$ $j \leq m$, form a cover of $D(\mathbf{0} ; r) \backslash\{\boldsymbol{x} ; P(\boldsymbol{x})=0\}$. Given $f_{j}: \Pi_{j} \rightarrow \mathbb{C}$, $j=1, \ldots, m$, bounded and analytic, assume that there exists $\gamma>0$ such that for every couple $\left(j_{1}, j_{2}\right)$

$$
\left|f_{j_{1}}(\boldsymbol{x})-f_{j_{2}}(\boldsymbol{x})\right|=O\left(\exp \left(-\gamma /|P(\boldsymbol{x})|^{1 / s}\right)\right)
$$

for $\boldsymbol{x} \in \Pi_{j_{1}} \cap \Pi_{j_{2}}$, provided $\Pi_{j_{1}} \cap \Pi_{j_{2}} \neq \emptyset$. Then the functions $f_{j}$ have $P$-s-Gevrey asymptotic expansions with a common right hand side.

Proof: Consider some injective linear functional $\ell: \mathbb{N}^{d} \rightarrow \mathbb{R}_{+}$and the operators $T_{\ell}$ corresponding to it for the $P$-sectors $\Pi_{j}$ and their nonempty intersections according to Theorem 4.7. Here the fact that the operators are independent of the $P$-sector in the sense of Remark 4.8 (3) is important. Without loss of generality, we can assume that the constants $\rho, \sigma, L$ of that theorem are the same for all these finitely many sectors. For convenience, we identify the Banach spaces $\mathcal{O}_{b}(V(a, b ; \sigma) \times D(\mathbf{0} ; \rho), \mathbb{C})$ with $\mathcal{O}_{b}\left(V(a, b ; \sigma), \mathcal{O}_{b}(D(\mathbf{0} ; \rho), \mathbb{C})\right)$ in the usual way. The inequalities (7.4) imply that

$$
\left\|\left(T_{\ell} f_{j_{1}}\right)(t)-\left(T_{\ell} f_{j_{2}}\right)(t)\right\|_{\mathcal{O}_{b}(D(\mathbf{0} ; \rho), \mathbb{C})}=O\left(\frac{1}{|t|} \exp \left(-\gamma|t|^{-1 / s}\right)\right)
$$

for $t \in V\left(a_{j_{1}}, b_{j_{1}} ; \sigma\right) \cap V\left(a_{j_{2}}, b_{j_{2}} ; r\right)$ provided this intersection is nonempty. Here the classical Ramis-Sibuya Theorem 3.4 applies and yields that the functions $T_{\ell} f_{j}$ have common $s$-Gevrey asymptotic expansions. We conclude using Theorem 7.3.

In the same way, the complement to the classical Ramis-Sibuya theorem (Theorem 3.5) will now be carried over to Gevrey asymptotics in a germ.

Theorem 7.19. Suppose that the P-sectors $\Pi_{j}=\Pi_{P}\left(a_{j}, b_{j} ; r\right), 1 \leq$ $j \leq m$, form a P-cover. For couples $\left(j_{1}, j_{2}\right)$ with $\Pi_{j_{1}} \cap \Pi_{j_{2}} \neq \emptyset$, let holomorphic $d_{j_{1}, j_{2}}: \Pi_{j_{1}} \cap \Pi_{j_{2}}: \rightarrow E$ be given that satisfy the cocycle condition $d_{j_{1}, j_{2}}+d_{j_{2}, j_{3}}=d_{j_{1}, j_{3}}$ whenever $\Pi_{j_{1}} \cap \Pi_{j_{2}} \cap \Pi_{j_{3}} \neq \emptyset$ and estimates

$$
\left|d_{j_{1}, j_{2}}(\boldsymbol{x})\right|=O\left(\exp \left(-\gamma /|P(\boldsymbol{x})|^{1 / s}\right)\right)
$$

for $j_{1}, j_{2} \in\{1, \ldots, m\}$ and $x \in \Pi_{j_{1}} \cap \Pi_{j_{2}}$ with some constants $s, \gamma>0$.

Then there exist $\rho>0$ and bounded holomorphic functions $f_{j}: \Pi_{j} \cap$ $D(\mathbf{0} ; \rho) \rightarrow E$ such that $\left.d_{j_{1}, j_{2}}\right|_{\Pi_{j_{1}} \cap \Pi_{j_{2}} \cap D(\mathbf{0} ; \rho)}=f_{j_{1}}-f_{j_{2}}$ whenever $\Pi_{j_{1}} \cap$ $\Pi_{j_{2}} \neq \emptyset$; moreover the functions $f_{j}$ have $P$-s-Gevrey asymptotic expansions with a common right hand side. 
Proof: With the same notation as before, take an injective linear functional $\ell: \mathbb{N}^{d} \rightarrow \mathbb{R}_{+}$, and the operators $T_{\ell}$. If $\Pi_{j_{1}} \cap \Pi_{j_{2}}=\Pi_{P}\left(a_{j_{1} j_{2}}, b_{j_{1} j_{2}} ; r\right)$, consider $T_{\ell} d_{j_{1} j_{2}}(t, \boldsymbol{x}) \in \mathcal{O}\left(V\left(a_{j_{1} j_{2}}, b_{j_{1} j_{2}} ; \sigma\right) \times D(\mathbf{0} ; \rho)\right)$, for some $\sigma, \rho$. If $\Pi_{j_{1}} \cap \Pi_{j_{2}} \cap \Pi_{j_{3}} \neq \emptyset$ then we have that $\left(T_{\ell} d_{j_{1} j_{2}}\right)(t, \boldsymbol{x})+\left(T_{\ell} d_{j_{2} j_{3}}\right)(t, \boldsymbol{x})=$ $\left(T_{\ell} d_{j_{1} j_{3}}\right)(t, \boldsymbol{x})$ because the operators $T_{\ell}$ are independent of the $P$-sector (Remark 4.8). The hypotheses of Theorem 3.5 are verified, and hence, there exist holomorphic bounded functions $F_{j}: V\left(a_{j}, b_{j} ; \sigma\right) \times D(\mathbf{0} ; \rho) \rightarrow$ $\mathbb{C}$ such that $F_{j_{1}}-F_{j_{2}}=T_{\ell} d_{j_{1} j_{2}}$ whenever $V\left(a_{j_{1}}, b_{j_{1}} ; \sigma\right) \cap V\left(a_{j_{2}}, b_{j_{2}} ; \sigma\right) \neq$ $\emptyset$. The functions $f_{j}(\boldsymbol{x})=F_{j}(P(\boldsymbol{x}), \boldsymbol{x})$ satisfy the statement of the theorem.

7.2. Summability with respect to a germ. In the sequel we still consider some analytic germ $P$ and suppose that $P \in \mathcal{O}_{b}(D(\mathbf{0}, R)) \backslash\{0\}$, $P(\mathbf{0})=0$. The existence of a summability result in a germ is based on the following Watson's lemma. It generalizes the theorem for monomial expansions (Theorem 3.15), and as before it is easily established by carrying over the classical version Theorem 3.1 (3) using Theorem 4.7.

Lemma 7.20. Let $\Pi=\Pi_{P}(a, b ; R)$ a sector in $P$ with $b-a>s \pi$ and suppose that $f \in \mathcal{O}(\Pi)$ has $\hat{f}=0$ as its $P$-s-Gevrey asymptotic expansion. Then $f \equiv 0$.

Now the following definition makes sense.

Definition 7.21. Let $\Pi=\Pi_{P}(a, b ; r)$ be a $P$-sector with $b-a>s \pi$, $k=\frac{1}{s}$, and $\hat{f} \in \hat{\mathcal{O}}$. We will say that $\hat{f}$ is $P$ - $k$-summable in $\Pi$ if there exists $f \in \mathcal{O}(\Pi)$ having $\hat{f}$ as $P$-s-Gevrey asymptotic expansion. In this situation, $f$ is called the $P$ - $k$-sum of $\hat{f}$ in $\Pi$ (and it is uniquely determined by Lemma 7.20).

$\hat{f}$ is called $P$ - $k$-summable in direction $\theta \in \mathbb{R}$ if there exists a $P$-sector $\Pi$ as before, bisected by $\theta$, with $b-a>s \pi$, and such that $\hat{f}$ is $P$ - $k$-summable in $\Pi$. $\hat{f}$ is called $P$ - $k$-summable if it is summable in every direction $\theta \in \mathbb{R}$ but a finite number $\bmod 2 \pi$.

The above notion of $P$ - $k$-summability in a direction $\theta$ does not indicate how to obtain a sum from a given series. Theorem 7.3 allows us to carry over the classical characterization using Laplace integrals (see Proposition 3.3) to the new concept.

Proposition 7.22. Let $s=1 / k$. Given a $P$-s-Gevrey series $\hat{f}(x) \in \hat{\mathcal{O}}$, it is $P$ - $k$-summable in a direction $\theta$ with $P$ - $k$-sum $f$ if and only if there exist $\rho>0$ and a formal Gevrey series $\hat{F}(t)=\sum_{n=0}^{\infty} g_{n} t^{n} \in \mathcal{O}_{b}(D(\mathbf{0} ; \rho)) \llbracket t \rrbracket_{s}$ with $\hat{F}(P(\boldsymbol{x}))(\boldsymbol{x}):=\sum_{n=0}^{\infty} J\left(g_{n}\right)(\boldsymbol{x}) J(P)(\boldsymbol{x})^{n}=\hat{f}(\boldsymbol{x})$ and the following properties: 
(1) The formal Borel transform $G(\boldsymbol{x}, \tau)=\sum g_{n}(\boldsymbol{x}) \tau^{n} / \Gamma(1+n / k)$ of $\hat{F}(t)$ is analytic in a neighborhood of the origin.

(2) The function $G$ can be continued analytically with to some domain $D(\mathbf{0} ; \rho) \times V(\theta-\delta, \theta+\delta ; \infty)$.

(3) It has exponential growth there, i.e. there are positive constants such that

$$
|G(\boldsymbol{x}, \tau)| \leq C \exp \left(A|\tau|^{k}\right) \quad \text { for }(\boldsymbol{x}, \tau) \in S ;
$$

so the Laplace integral $F(\boldsymbol{x}, t)=k t^{-k} \int_{\arg \tau=\tilde{\theta}} e^{-\tau^{k} / t^{k}} G(\boldsymbol{x}, \tau) \tau^{k-1} d \tau$ converges for $\boldsymbol{x} \in D(\mathbf{0} ; \rho)$ and $t$ in a certain sector $V=V\left(\theta-\frac{\pi}{2 k}-\right.$ $\left.\frac{\tilde{\delta}}{k}, \theta+\frac{\pi}{2 k}+\frac{\tilde{\delta}}{k} ; r\right), 0<\tilde{\delta}<\delta$, and suitably chosen $\tilde{\theta}$ close to $\theta$.

(4) Finally $f(\boldsymbol{x})=F(\boldsymbol{x}, P(\boldsymbol{x}))$ which has $\hat{f}(\boldsymbol{x})$ as its $P$-s-Gevrey asymptotic expansion on some $\Pi$-sector $\Pi_{P}\left(\theta-\frac{\pi}{2 k}-\frac{\tilde{\delta}}{k}, \theta+\frac{\pi}{2 k}+\frac{\tilde{\delta}}{k} ; \tilde{\rho}\right)$, $\tilde{\rho}>0$.

As the concepts of asymptotic expansion in a germ and Gevrey asymptotic expansions in a germ, the notion of summability with respect to a germ behaves well under blow-ups. We first show:

Proposition 7.23. Consider a P-sector $\Pi=\Pi_{P}(a, b ; r)$ with $b-a>s \pi$, and $\hat{f} \in \hat{\mathcal{O}}$, such that for every $\xi \in \mathbb{P}_{\mathbb{C}}^{1}$, the series $\hat{g}_{\xi}=\hat{f} \circ b_{\xi}$ is $P \circ b_{\xi}$ $k$-summable in $\Pi_{\xi}:=\Pi_{P \circ b_{\xi}}\left(a, b ; r_{\xi}\right)$. Then, $\hat{f}$ is P-k-summable in $\Pi$.

$A$ formal series $\hat{f} \in \hat{\mathcal{O}}$ is $P$ - $k$-summable in a direction $d$ if and only if for every $\xi \in \mathbb{P}_{\mathbb{C}}^{1}, \hat{f} \circ b_{\xi}$ is $P \circ b_{\xi}$-k-summable in direction $d$.

Proof: We only prove the second statement; the proof of the first is analogous. Also, we only prove the nontrivial implication.

Suppose that for every $\xi \in \mathbb{P}_{\mathbb{C}}^{1}$, the series $\hat{f} \circ b_{\xi}$ is $P \circ b_{\xi}-k$-summable on some $P \circ b_{\xi}$-sector $\Pi_{\xi}=\Pi_{P \circ b_{\xi}}\left(d-\varphi_{\xi}, d+\varphi_{\xi} ; r_{\xi}\right)$ with $\varphi_{\xi}>\pi / k$. This means that for every $\xi \in \mathbb{P}_{\mathbb{C}}^{1}$, there exist uniquely determined functions $g_{\xi}: \Pi_{\xi} \rightarrow \mathbb{C}$ that have $\hat{f} \circ b_{\xi}$ as their $P \circ b_{\xi}-s$-Gevrey asymptotic expansion, $s=1 / k$.

With the notation of Subsection 2.2, let $U_{\xi}=\phi_{\xi}^{-1}\left(D\left(\mathbf{0}, \rho_{\xi}\right)\right) \subset M$, a neighborhood of $(\xi, \mathbf{0}) \in M$. Then $G_{\xi}=g_{\xi} \circ \phi_{\xi}$ are defined on $U_{\xi} \cap\{p \mid$ $\left.|\arg (P(b(p)))-d|<\varphi_{\xi}\right\}$.

Observe that by Lemma 7.8, the composition $g_{\xi} \circ\left(\phi_{\xi} \circ \phi_{\chi}^{-1}\right)$ has the $P \circ b_{\chi}-s$-Gevrey asymptotic expansion $\hat{f} \circ b_{\chi}$ on $\Pi_{P \circ b_{\chi}}\left(d-\varphi_{\xi}, d+\varphi_{\xi}, \mu\right)$ and hence it is the $P \circ b_{\chi}-k$-sum of $\hat{f} \circ b_{\chi}$ on this sector for some small $\mu$ if $\chi$ is sufficiently close to $\xi$. Without loss of generality we can assume 
that this is already the case for $\chi \in U_{\xi}$. By Watson's Lemma 7.20, we then have

$$
g_{\xi} \circ\left(\phi_{\xi} \circ \phi_{\chi}^{-1}\right)(\mathbf{v})=g_{\chi}(\mathbf{v})
$$

for $\chi \in U_{\xi}$ and small $\mathbf{v}$ with $\left|\arg \left(P \circ b_{\chi}\right)(\mathbf{v})-d\right|<\min \left(\varphi_{\xi}, \varphi_{\chi}\right)$.

Using this property with some $\chi \in U_{\xi} \cap U_{\zeta}$ and extending it because of the analyticity of the functions, we obtain

$$
\begin{aligned}
& G_{\xi}(p)=G_{\zeta}(p) \\
& \quad \text { for } \xi, \zeta \in \mathbb{P}_{\mathbb{C}}^{1}, p \in U_{\xi} \cap U_{\zeta} \text { with }|\arg (P(b(p)))-d|<\min \left(\varphi_{\xi}, \varphi_{\zeta}\right) .
\end{aligned}
$$

Using the compactness of $\mathbb{P}_{\mathbb{C}}^{1}$ as before, a finite number of members of the family $U_{\xi}, \xi \in \mathbb{P}_{\mathbb{C}}^{1}$, covers the exceptional divisor $E=\mathbb{P}_{\mathbb{C}}^{1} \times\{\mathbf{0}\}$ in $M$, say $E \subset U=\cup_{i=1, \ldots, n} U_{\xi_{i}}$. Then property (7.7) allows us to define $G$ for $p \in U$ with $|\arg (P(b(p)))-d|<\varphi=\min _{i} \varphi_{\xi_{i}}$ by $G(p)=G_{\xi}(p)$ if $p \in U_{\xi},|\arg (P(b(p)))-d|<\varphi$. In turn, we obtain a function $g$ defined for small $\boldsymbol{x} \in \mathbb{C}^{d}$ with $|\arg (P(\boldsymbol{x}))-d|<\varphi$ by setting $g(b(p))=G(p)$.

Then $g$ has $\hat{f}$ as $P$-s-Gevrey asymptotic expansion on $\Pi_{P}(d-\varphi, d+$ $\varphi ; \rho)$ for sufficiently small positive $\rho$. Indeed, using property (7.7), we find that for every $\xi \in \mathbb{P}_{\mathbb{C}}^{1}$, the function $g \circ b_{\xi}=G_{\xi} \circ \phi_{\xi}^{-1}$ is the restriction of $g_{\xi}$ to $\Pi_{P \circ b_{\xi}}(d-\varphi, d+\varphi ; \rho)$ and it has $\hat{f} \circ b_{\xi}$ as its $P \circ b_{\xi}-s$-Gevrey asymptotic expansion. By Theorem 7.6, $g$ has some $P$-s-Gevrey asymptotic expansion $\hat{g}$ on $\Pi_{P}(d-\varphi, d+\varphi ; \rho)$. Obviously, we have $\hat{g} \circ b_{\xi}=\hat{f} \circ b_{\xi}$ for every $\xi \in \mathbb{P}_{\mathbb{C}}^{1}$ and thus $\hat{g}=\hat{f}$. This means that $g$ is the $P$ - $k$-sum of $\hat{f}$ in direction $d$.

As for Propositions 7.6 and 7.9, the above proposition can be improved; again, it is sufficient to assume summability of $\hat{f} \circ b_{\xi}$ for a finite number of well chosen $\xi$.

Theorem 7.24. Consider $\mathcal{L}, \ell, h, H_{\mathcal{L}}, \mathcal{Z}_{\mathcal{L}}$, and $\mathcal{Z}$ as in Theorem 7.7. Let $\hat{f} \in \hat{\mathcal{O}}$ be given such that $\hat{f} \circ b_{\xi}$ is $P \circ b_{\xi}-k$-summable in the direction $d$ for $\xi \in \mathcal{Z}$. Then $\hat{f}$ is also $P$-k-summable in direction $d$.

Corollary 7.25. Consider $\mathcal{L}, \ell, h, H_{\mathcal{L}}, \mathcal{Z}_{\mathcal{L}}$, and $\mathcal{Z}$ as above and $\hat{f} \in \hat{\mathcal{O}}$. If $\hat{f} \circ b_{\xi}$ is $P \circ b_{\xi}-k$-summable for $\xi \in \mathcal{Z}$ then $\hat{f}$ is $P$ - $k$-summable.

Proof: By the theorem, the singular directions of $\hat{f}$, i.e. the directions $d$ for which it is not $P$ - $k$-summable, are contained in the finite union of the sets of exceptional directions for $\hat{f} \circ b_{\xi}, \xi \in \mathcal{Z}$. 
Proof of the theorem: We can again assume that $\infty \in \mathcal{Z}$; otherwise we proceed as in the beginning of the proof of Theorem 6.8.

As summable series are also Gevrey, application of Theorem 7.10 yields that $\hat{f}$ is a $P$-s-Gevrey series, $s=1 / k$. As a consequence $\hat{f} \circ b_{\xi}$ are $P \circ b_{\xi^{-}}-$-Gevrey series for every $\xi \in \mathbb{P}_{\mathbb{C}}^{1}$.

By Lemma 6.9 there exists a formal series $\hat{G}(t, \mathbf{v})=\sum_{n=0}^{\infty} \hat{g}_{n}(\mathbf{v}) t^{n} \in$ $\mathcal{B} \llbracket t \rrbracket, \mathcal{B}=\mathbb{C}\left[v_{1}, 1 / H_{\mathcal{L}}\left(1, v_{1}\right)\right] \llbracket \mathbf{v}^{\prime} \rrbracket$ with the following property. For all $\xi \in$ $\mathbb{C} \backslash \mathcal{Z}_{\mathcal{L}}$, the series

$$
T_{\ell}\left(\hat{f} \circ b_{\xi}\right)(t, \mathbf{v})=\sum_{n=0}^{\infty} \hat{f}_{n \xi}(\mathbf{v}) t^{n} \in \Delta_{\ell}\left(P \circ b_{\xi}\right) \llbracket t \rrbracket
$$

of Lemma 4.6 applied with $P \circ b_{\xi}$ in place of $P$ satisfies

$$
\hat{f}_{n \xi}(\mathbf{v})=J_{\xi}\left(\hat{g}_{n}\right)(\mathbf{v}) \quad \text { for } n \in \mathbb{N} .
$$

Since all $\hat{f} \circ b_{\xi}$ are $P \circ b_{\xi^{-}}$-Gevrey series, we obtain from Definition/ Proposition 7.5 that the series $\hat{f}_{n, \xi}(\mathbf{v})$ are convergent for all $n$ and $\xi \in$ $\mathbb{C} \backslash \mathcal{Z}_{\mathcal{L}}$, that for every $\xi \in \mathbb{C} \backslash \mathcal{Z}_{\mathcal{L}}$ there exists $\rho_{\xi}>0$ such that $\hat{f}_{n, \xi}(\mathbf{v})$ defines an element of $\mathcal{O}_{b}\left(D\left(\mathbf{0}, \rho_{\xi}\right)\right)$ that we denote $f_{n, \xi}$ and there exist $K_{\xi}, A_{\xi}>0$ such that for all $n$

$$
\left|f_{n, \xi}(\mathbf{v})\right| \leq K_{\xi} A_{\xi}^{n} \Gamma(s n+1) \quad \text { for }|\mathbf{v}|<\rho_{\xi} .
$$

We can now define $g_{n}$ by $g_{n}(\mathbf{v})=f_{n \xi}\left(v_{1}-\xi, \mathbf{v}^{\prime}\right)$ if $\left|v_{1}-\xi\right|$ and $\left|\mathbf{v}^{\prime}\right|$ are sufficiently small. The value of $g_{n}(\mathbf{v})$ does not depend on $\xi$ because of (7.8). This defines functions $g_{n}$ on some common neighborhood $\Omega$ of $\left(\mathbb{C} \backslash \mathcal{Z}_{\mathcal{L}}\right) \times\{\mathbf{0}\} \subset \mathbb{C}^{d}$. By (7.9), the formal Borel transform $\tilde{G}(\tau, \mathbf{v})=\sum_{n=0}^{\infty} g_{n}(\mathbf{v}) \tau^{n} / \Gamma(s n+1)$ defines a holomorphic function $\tilde{G}$ on some neighborhood of $\Omega \times\{0\}$ in $\mathbb{C}^{d+1}$.

By assumption and Lemma 7.8, $\hat{f} \circ b_{\xi}$ is $P \circ b_{\xi}$-summable for all $\xi$ large and $\xi$ close to $\mathcal{Z}^{\prime}=\mathcal{Z} \backslash\{\infty\}$. Consider again the domain $\mathcal{D}=$ $D(0, R) \backslash \cup_{\chi \in \mathcal{Z}^{\prime}} \operatorname{cl}(D(\chi, r))$ where $r, R$ were chosen such that summability holds for $|\xi|>R / 2$ or $\operatorname{dist}\left(\xi, \mathcal{Z}^{\prime}\right)<2 r$. By Proposition 7.22, (7.8), and compactness, we can find positive $\rho, \delta$ such that $\tilde{G}(\tau, \mathbf{v})$ can be continued analytically to the set $W \times A$ where $W$ is the set of all $\tau$ with $\tau \in D(0, \rho) \cup V(d-\delta, d+\delta, \infty), A$ the union of "tori" of all $\mathbf{v}$ with $\operatorname{dist}\left(v_{1}, \partial \mathcal{D}\right)<\rho,\left|\mathbf{v}^{\prime}\right|<\rho$. Also by Proposition $7.22, \tilde{G}$ has at most exponential growth as $\tau \rightarrow \infty$ : There exist $K, L$ such that $|\tilde{G}(\tau, \mathbf{v})| \leq$ $K \exp \left(L|\tau|^{k}\right)$ for $\mathbf{v} \in A, \tau \in W,|\tau| \geq 1$.

We can assume that also the set $\Omega^{\prime}$ of all $\mathbf{v}$ with $\operatorname{dist}\left(v_{1}, \mathcal{D}\right)<\rho$ and $\left|\mathbf{v}^{\prime}\right|<\rho$ is contained in $\Omega$ and that $\tilde{G}$ is holomorphic on $D(0, \rho) \times \Omega^{\prime}$. Now $\tilde{G}$ is holomorphic on the union of $W \times A$ and of $D(0, \rho) \times \Omega^{\prime}$. This 
is a "U-shaped" domain and hence Hartogs' lemma can be applied. It yields that $\tilde{G}$ can be continued analytically to $W \times \Omega^{\prime}$.

For the convenience of the reader we give a short proof using Cauchy's formula. For $\tau \in D(0, \rho)$ and $\mathbf{v} \in \Omega^{\prime}$, we have

$$
2 \pi i \tilde{G}(\tau, \mathbf{v})=\left(\oint_{|z|=R+\tilde{\rho}}-\sum_{\chi \in \mathcal{Z}^{\prime}} \oint_{|z-\chi|=r-\tilde{\rho}}\right) \frac{\tilde{G}\left(\tau,\left(z, \mathbf{v}^{\prime}\right)\right)}{z-v_{1}} d z
$$

for $\tilde{\rho} \in] 0, \rho[$ sufficiently close to $\rho$ (more precisely, we must have $\rho>\tilde{\rho}>$ $\left.\operatorname{dist}\left(v_{1}, \mathcal{D}\right)\right)$. As the right hand side of (7.10) only uses values $\tilde{G}\left(\tau,\left(z, \mathbf{v}^{\prime}\right)\right)$ where $\left(z, \mathbf{v}^{\prime}\right) \in A$, it is defined for any $\tau \in W$, hence the right hand side can be continued to an analytic function on $W \times \Omega^{\prime}$. As they coincide except for a constant factor on some open subset of $W \times \Omega^{\prime}$, the same is true for $\tilde{G}$.

Now the maximum modulus principle applied in the variable $v_{1}$ permits to carry over the exponential estimate of $\tilde{G}$ to $W \times \Omega^{\prime}$ : we have $|\tilde{G}(\tau, \mathbf{v})| \leq K \exp \left(L|\tau|^{k}\right)$ for $\mathbf{v} \in \Omega^{\prime}$ and $\tau \in W,|\tau| \geq 1$.

As before, this implies that $\hat{f} \circ b_{\xi}$ is $P \circ b_{\xi^{-}}$-summable in direction $d$ for all $\xi \in \mathcal{D}$. As this is already known for the remaining $\xi \in \mathbb{P}_{\mathbb{C}}^{1}$, we have it for all $\xi \in \mathbb{P}_{\mathbb{C}}^{1}$. We conclude with Proposition 7.23.

As for $P$ - and $P$-s-Gevrey asymptotic expansion, we complete the theory of $P$ - $k$-summability with statements concerning the compatibility with ramification.

Proposition 7.26. Consider a $P$-sector $\Pi=\Pi_{P}(a, b ; r)$ with $b-a>s \pi$, $k=1 / s, \hat{f} \in \hat{\mathcal{O}}$, and a positive integer $m$.

If the series $\hat{g}_{m}=\hat{f} \circ r_{m}$ is $P \circ r_{m}-k$-summable in $\Pi_{m}:=\Pi_{P \circ r_{m}}(a, b ; \tilde{r})$ then $\hat{f}$ is $P$-k-summable in $\Pi$.

$\hat{f}$ is $P$-k-summable in some direction d if and only if $\hat{f} \circ r_{m}$ is $P \circ r_{m^{-}}$ $k$-summable in direction $d$.

$\hat{f}$ is $P$-k-summable if and only if $\hat{f} \circ r_{m}$ is $P \circ r_{m}-k$-summable.

Proof: The first statement follows immediately from the definition of summability in a sector and Proposition 7.13. The remaining two then follow from the definitions of summability in a direction respectively summability.

7.3. Consequences and further properties. Theorem 7.18 may be used, as in classical asymptotics, to show properties about the composition of functions having $P$ - $s$-Gevrey asymptotic expansion, and analytic functions. More precisely: 
Theorem 7.27. Consider $P(\boldsymbol{x})$ as before, with $P(\mathbf{0})=0$, and $\Pi=$ $\Pi_{P}(a, b ; r)$ a P-sector. Let $f_{1}(\boldsymbol{x}), \ldots, f_{n}(\boldsymbol{x}) \in \mathcal{O}(\Pi)$ be functions having series $\hat{f}_{1}(\boldsymbol{x}), \ldots, \hat{f}_{n}(\boldsymbol{x})$, respectively, as $P$ - $s_{i}$-asymptotic expansions, $i=$ $1, \ldots, n$, with $\hat{f}_{i}(\mathbf{0})=0$. Let $D$ be a disk around the origin in $\mathbb{C}^{d+n}$, and $F(\boldsymbol{x}, \mathbf{y})=F\left(x_{1}, \ldots, x_{d}, y_{1}, \ldots, y_{n}\right) \in \mathcal{O}(D)$. Then, if $s=\max \left\{s_{1}, \ldots, s_{n}\right\}$, we have:

(1) $F\left(\boldsymbol{x}, f_{1}(\boldsymbol{x}), \ldots, f_{n}(\boldsymbol{x})\right)$ is defined in a P-sector $\tilde{\Pi}=\Pi_{P}(a, b ; \tilde{r})$, with $\tilde{r} \leq r$ small enough.

(2) $F\left(\boldsymbol{x}, f_{1}(\boldsymbol{x}), \ldots, f_{n}(\boldsymbol{x})\right)$ has a $P$-s-Gevrey asymptotic expansion in $\tilde{\Pi}$.

Proof: The conditions $\hat{f}_{i}(\mathbf{0})=0$ imply that $\lim _{\Pi \ni \boldsymbol{x} \rightarrow \mathbf{0}} f_{i}(\boldsymbol{x})=0$ and the first statement follows. As a $P-s_{i}$-Gevrey asymptotic expansion also is a $P$-s-Gevrey asymptotic expansion, we can assume that all $s_{i}=s$. For simplicity of notation, we combine $\boldsymbol{f}(\boldsymbol{x})=\left(f_{1}(\boldsymbol{x}), \ldots, f_{n}(\boldsymbol{x})\right), \hat{\boldsymbol{f}}(\boldsymbol{x})=$ $\left(\hat{f}_{1}(\boldsymbol{x}), \ldots, \hat{f}_{n}(\boldsymbol{x})\right)$. By Proposition 7.17 , there exist a $P$-cover $\{\Pi=$ $\left.\Pi_{1}, \Pi_{2}, \ldots, \Pi_{r}\right\}$, with $\Pi_{i}=\Pi_{P}\left(a_{i}, b_{i} ; \tilde{r}\right)$, and functions $\boldsymbol{f}_{i} \in \mathcal{O}\left(\Pi ; \mathbb{C}^{n}\right)$, $1 \leq i \leq r$, such that $\boldsymbol{f}_{i}(\boldsymbol{x})$ has $\hat{\boldsymbol{f}}(\boldsymbol{x})$ as $P$-s-asymptotic expansion. Consider the functions $g_{i}(\boldsymbol{x})=F(\boldsymbol{x}, \boldsymbol{f}(\boldsymbol{x}))$, defined on $\Pi_{P}\left(a_{i}, b_{i} ; \tilde{r}\right)$, reducing $\tilde{r}$ again if necessary. If $\Pi_{j_{1}} \cap \Pi_{j_{2}} \neq \emptyset$ then

$$
\begin{aligned}
\left(g_{j_{1}}-g_{j_{2}}\right)(\boldsymbol{x})= & \boldsymbol{H}(\boldsymbol{x})\left(\boldsymbol{f}_{j_{1}}-\boldsymbol{f}_{j_{2}}\right)(\boldsymbol{x}), \\
& \text { where } \boldsymbol{H}(\boldsymbol{x})=\int_{0}^{1} \frac{\partial F}{\partial \mathbf{y}}\left(\boldsymbol{x}, \tau \boldsymbol{f}_{j_{1}}(\boldsymbol{x})+(1-\tau) \boldsymbol{f}_{j_{2}}(\boldsymbol{x})\right) d \tau .
\end{aligned}
$$

As with $k=1 / s$

$$
\left|\boldsymbol{f}_{j_{1}}(\boldsymbol{x})-\boldsymbol{f}_{j_{2}}(\boldsymbol{x})\right| \leq K \exp \left(-\frac{A}{|P(\boldsymbol{x})|^{k}}\right)
$$

for certain $K, A>0$, we obtain that

$$
\left|g_{j_{1}}(\boldsymbol{x})-g_{j_{2}}(\boldsymbol{x})\right| \leq \tilde{K} \exp \left(-\frac{A}{|P(\boldsymbol{x})|^{k}}\right)
$$

for appropriate $\tilde{K}>0$, reducing radii if necessary. These estimates and Theorem 7.18 show that every $g_{i}(\boldsymbol{x}), i=1, \ldots, r$, has a $P$-s-asymptotic expansion, and the result follows.

Remark 7.28. This result provides an alternative proof that the product of functions having $P$-s-Gevrey asymptotic expansions has a $P$-s-Gevrey asymptotic expansion. Indeed, just take $F\left(\boldsymbol{x}, y_{1}, y_{2}\right)=y_{1} y_{2}$ in the above theorem.

Concerning the partial derivatives of a function having $P$-s-Gevrey asymptotic expansion, we can proceed as above first embedding the 
function in a $P$ - $k$-quasifunction using Proposition 7.17 and applying the Ramis-Sibuya Theorem 7.18 to the derivatives. For this approach, we have to show that, if $f(\boldsymbol{x}) \in \mathcal{O}\left(\Pi_{P}\right)$ verifies an estimate

$$
|f(\boldsymbol{x})| \leq C \exp \left(-\frac{A}{|P(\boldsymbol{x})|^{k}}\right)
$$

then their derivatives satisfy similar estimates.

To show this, consider $\Pi_{P}=\Pi_{P}(a, b ; r), f \in \mathcal{O}\left(\Pi_{p}\right)$ verifying (7.11). Lemma 5.4 shows the existence of a bounded holomorphic function $F$ : $V(a, b ; \sigma) \times D(\mathbf{0} ; \rho) \rightarrow \mathbb{C}$ with $F(P(\boldsymbol{x}), \boldsymbol{x})=f(\boldsymbol{x})$ and

$$
F(t, \boldsymbol{x}) \leq \frac{C L}{|t|} \exp \left(-\frac{A}{|t|^{k}}\right) .
$$

Choosing some positive $A^{\prime}<A$, there exist $C^{\prime}>0$ such that

$$
|F(t, \boldsymbol{x})| \leq C^{\prime} \exp \left(-\frac{A^{\prime}}{|t|^{k}}\right) .
$$

Taking derivatives, we obtain

$$
\frac{\partial f}{\partial x_{i}}(\boldsymbol{x})=\frac{\partial F}{\partial t}(P(\boldsymbol{x}), \boldsymbol{x}) \frac{\partial P}{\partial x_{i}}(\boldsymbol{x})+\frac{\partial F}{\partial x_{i}}(P(\boldsymbol{x}), \boldsymbol{x}) .
$$

Now choose $a<\alpha<\beta<b, 0<\sigma^{\prime}<\sigma$, and $0<\rho^{\prime}<\rho$. Applying Cauchy's formula for the derivative of a holomorphic function, we obtain in a well known way the existence of certain positive $D, B$ such that

$$
\left|\frac{\partial F}{\partial t}(t, \boldsymbol{x})\right| \leq D \exp \left(-\frac{B}{|t|^{k}}\right) \quad \text { and } \quad\left|\frac{\partial F}{\partial x_{i}}(t, \boldsymbol{x})\right| \leq D \exp \left(-\frac{B}{|t|^{k}}\right)
$$

for $(t, \boldsymbol{x}) \in V\left(\alpha, \beta ; \sigma^{\prime}\right) \times D\left(\mathbf{0} ; \rho^{\prime}\right)$. We obtain the existence of positive $\tilde{C}$, $\tilde{A}$ such that

as needed. Thus we have proved:

$$
\left|\frac{\partial f}{\partial x_{i}}(\boldsymbol{x})\right| \leq \tilde{C} \exp \left(-\frac{\tilde{A}}{|P(\boldsymbol{x})|^{k}}\right) .
$$

Proposition 7.29. If $\Pi=\Pi_{P}(a, b ; r)$ is a P-sector and $f \in \mathcal{O}_{b}(\Pi)$ has a P-s-Gevrey asymptotic expansion, then for $\alpha, \beta$ such that $a<\alpha<$ $\beta<b, \frac{\partial f}{\partial x_{i}}(\boldsymbol{x})$ has a $P$-s-Gevrey asymptotic expansion in $\Pi_{P}(\alpha, \beta ; r)$.

\section{Examples}

8.1. First example. We consider the following singular ordinary differential equation depending upon a small parameter $\varepsilon$

$$
P(x, \varepsilon)^{2} \frac{d y}{d x}=\left(P^{\prime}(x, \varepsilon)+A(x, \varepsilon)\right) y+B(x, \varepsilon)+y^{2} f(x, \varepsilon, y),
$$

where $P(x, \varepsilon)=x^{n}+\cdots$ is a homogeneous polynomial having $n \geq 2$ simple roots if $\varepsilon \neq 0, P^{\prime}=\frac{\partial P}{\partial x}, A, B, f$ are holomorphic near the origin in $\mathbb{C}^{2}$ resp. $\mathbb{C}^{3}, A$ has a homogeneous valuation $w(A) \geq n$ and $w(B) \geq 2 n-1$ if $f$ is not identically 0 . If $\varepsilon \neq 0$ then (8.1) has $n$ finite irregular singular 
points of Poincaré rank 1. If $\varepsilon=0$, then $x=0$ is an irregular singular point of Poincaré rank $n$ after the reduction $y=x^{n-1} z$. This means that our equation has $n$ coalescing irregular singular points.

Theorem 8.1. Suppose that (8.1) has a formal solution $y(x, \varepsilon) \in \mathbb{C} \llbracket x, \varepsilon \rrbracket$. Then it is $P$-1-summable in every direction $d \not \equiv 0 \bmod 2 \pi \mathbb{Z}$.

Remark 8.2. The existence of such a formal solution is quite exceptional. It is nevertheless possible to have a divergent formal solution, as the simple example $y=\sum_{m=0}^{\infty} m ! P^{m+1}$ solution of $P^{2} \frac{d y}{d x}=P^{\prime} y-P P^{\prime}$ shows.

Proof: We apply Theorem 7.24 with $x_{1}$ replaced by $x, x_{2}$ replaced by $\varepsilon$. Then the set $\mathcal{Z}$ is the set of zeroes of the polynomial $P(u, 1)$ - it contains exactly $n$ elements. We have to show that $y \circ b_{\xi}$ is $P \circ b_{\xi}-1$-summable in every direction $d \not \equiv 0 \bmod 2 \pi \mathbb{Z}$ for any $\xi \in \mathcal{Z}$.

For simplicity, we consider only the case $\xi=0 \in \mathcal{Z}$; the modification for arbitrary $\xi \in \mathcal{Z}$ is left to the reader. Here, we perform the blowup $x=\varepsilon u$ and at the same time change the dependent variable by putting $y=\varepsilon^{n-1} z$. We obtain the doubly singular equation

$$
\varepsilon^{n} u^{2} \frac{d z}{d u}=\frac{1}{Q(u)^{2}}\left(\left(P^{\prime}(u, 1)+\varepsilon \tilde{A}(u, \varepsilon)\right) z+\varepsilon \tilde{B}(u, \varepsilon)+z^{2} f\left(\varepsilon u, \varepsilon, \varepsilon^{n-1} z\right)\right) \text {, }
$$

where $Q(u)=P(u) / u$ is a polynomial satisfying $Q(0)=P^{\prime}(0,1)$ and $\tilde{A}, \tilde{B}$ are analytic near the origin. (As there exists a formal solution, we can assume that $w(B) \geq 2 n-1$ also in the linear case.)

The main result of $[\mathbf{C M S}]$ applies to this equation and yields that its unique formal solution $z(u, \varepsilon)$ is $\varepsilon^{n} u$-1-summable in every direction not in $-\arg \left(P^{\prime}(0,1)\right) \bmod 2 \pi \mathbb{Z}$. It remains to multiply $\varepsilon^{n} u$ by the unit $Q(u)$. Observing that $Q(0)=P^{\prime}(0,1)$ we obtain the $z(u, \varepsilon)$ is $\varepsilon^{n} P(u, 1)$-1-summable for every direction not in $2 \pi \mathbb{Z}$.

8.2. Second example. We consider the following singular partial differential equation

$$
\left(x_{2} \frac{\partial P}{\partial P x_{2}}+\alpha P^{k+1}+P A\right) x_{1} \frac{\partial f}{\partial x_{1}}-\left(x_{1} \frac{\partial P}{\partial x_{1}}+\beta P^{k+1}+P B\right) x_{2} \frac{\partial f}{\partial x_{2}}=h
$$

where $P$ is a quasi-homogeneous polynomial for the valuation $w$ determined by $w\left(x_{1}\right)=a, w\left(x_{2}\right)=b$, where $k \in \mathbb{N}^{*}, h, A, B$ are convergent power series and $w(A), w(B)>k g, g=w(P)$.

Theorem 8.3. If (8.3) has a formal solution $f(\boldsymbol{u}) \in \mathbb{C} \llbracket \boldsymbol{u} \rrbracket$ then it is $P$-k-summable provided $\alpha, \beta$ satisfy the following conditions.

(1) $\alpha+\beta \neq 0$ if $P$ is not a monomial.

(2) $a \mu_{0} \beta \neq\left(g-b \mu_{0}\right) \alpha$ if $x_{2}$ is a factor of $P$ of multiplicity $\mu_{0}>0$.

(3) $b \mu_{\infty} \alpha \neq\left(g-a \mu_{\infty}\right) \beta$ if $x_{1}$ is a factor of $P$ of multiplicity $\mu_{\infty}>0$. 
Remark 8.4. (1) The existence of a formal solution is a very strong hypothesis, comparable to the necessary condition (see [CE, Lak]) for Ackerberg-O'Malley resonance [AO]. The investigation of conditions for the existence of formal solutions, also for non-linear equations, will be done in a future article.

(2) A formal solution of (8.3) is not necessarily convergent, as the simple example $f(\boldsymbol{x})=x_{1} \sum_{n=0}^{\infty} n ! P(\boldsymbol{x})^{n+1}$ with any quasi-homogeneous polynomial $P$ shows. It is solution of (8.3) with $k=1, \alpha=0, \beta=1$, $A=B=0$, and $h(\boldsymbol{x})=x_{2} \frac{\partial P}{\partial x_{2}} P$.

(3) In the case of a monomial $P$, equations like (8.3) have been studied by Pingli Li $[\mathbf{L i}]$ using a different method.

Proof: In a first step, we prove the statement in the case of monomials $P$. Then we reduce the statement for homogeneous and then quasihomogeneous polynomials $P$ to the former one using Theorem 7.24 and Proposition 7.26.

In the case of a monomial $P=x_{1}^{c} x_{2}^{d}, c, d$ positive integers, the choice of $a$ and $b$ is arbitrary. The conditions (2) and (3) are both equivalent to the condition that $\alpha / d \neq \beta / c$. The right hand side of equation (8.3), moreover, is divisible by $P$, because the left hand side is. We obtain the equation

$$
\left(d+\alpha P^{k}+A\right) x_{1} \frac{\partial f}{\partial x_{1}}-\left(c+\beta P^{k}+B\right) x_{2} \frac{\partial f}{\partial x_{2}}=h / P .
$$

For later use, it is convenient to relax the conditions on $A, B$ slightly. We we also allow terms in $A, B$ that are divisible by $x_{1}^{k c+1}$ or $x_{2}^{k d+1}$. Let $\mathcal{I}$ denote the ideal of all convergent series satisfying this condition.

We can now apply a result of Martinet and Ramis [MR] and obtain a formal change of coordinates $\boldsymbol{x}=\tilde{\boldsymbol{x}}+\psi(\tilde{\boldsymbol{x}})$ where both components of $\psi$ are in $\mathcal{I}$ such that the vector field

$$
\left(d+\alpha P^{k}+A\right) x_{1} \frac{\partial}{\partial x_{1}}-\left(c+\beta P^{k}+B\right) x_{2} \frac{\partial}{\partial x_{2}}
$$

in (8.4) is reduced its formal normal form $\left(d+\alpha P^{k}\right) \tilde{x}_{1} \frac{\partial}{\partial \tilde{x}_{1}}-\left(c+\beta P^{k}\right) \tilde{x}_{2} \frac{\partial}{\partial \tilde{x}_{2}}$ except for a unit factor $1+\phi(\tilde{\boldsymbol{x}}) \in 1+\mathcal{I}$. It is proved in [MR], furthermore, that $\phi$ and the components of $\psi$ are $P$ - $k$-summable under our condition $\alpha / d \neq \beta / c$.

It remains to solve a partial differential equation

$$
\left(d+\alpha P^{k}\right) x_{1} \frac{\partial f}{\partial x_{1}}-\left(c+\beta P^{k}\right) x_{2} \frac{\partial f}{\partial x_{2}}=h,
$$

where $P=x_{1}^{c} x_{2}^{d}$ and $h$ is a $P$-k-summable power series such that (8.5) admits a formal solution $f$. 
If $c>1$ then replacing $x_{1}$ by $x_{1} e^{2 \pi i / c}$ we find that $\tilde{f}\left(x_{1}, x_{2}\right)=$ $f\left(x_{1} e^{2 \pi i / c}, x_{2}\right)$ is a solution of (8.5) with right hand side $\tilde{h}\left(x_{1}, x_{2}\right)=$ $h\left(x_{1} e^{2 \pi i / c}, x_{2}\right)$. This leads us to split $f$ and $h$ into $c$ series: $f\left(x_{1}, x_{2}\right)=$ $\sum_{j=0}^{c-1} x_{1}^{j} f_{j}\left(x_{1}^{c}, x_{2}\right)$ and a similar formula for $h$ and $h_{j}, j=0, \ldots, c-1$. We obtain $c$ equations for the $f_{j}=f_{j}\left(u_{1}, x_{2}\right)$.

$$
\begin{array}{r}
\left(d+\alpha \bar{P}^{k}\right) u_{1} \frac{\partial f_{j}}{\partial u_{1}}-\left(1+\frac{\beta}{c} \bar{P}^{k}\right) x_{2} \frac{\partial f_{j}}{\partial x_{2}}+\frac{j}{c}\left(d+\alpha \bar{P}^{k}\right) f_{j}=\frac{1}{c} h_{j}, \\
j=0, \ldots, c-1,
\end{array}
$$

where $\bar{P}\left(u_{1}, x_{2}\right)=u_{1} x_{2}^{d}$. In a similar way, splitting

$$
f_{j}\left(u_{1}, x_{2}\right)=\sum_{m=0}^{d-1} x_{2}^{m} f_{j m}\left(u_{1}, x_{2}^{d}\right)
$$

we obtain equations for each of the $f_{j m}$. We simplify the notation by omitting the indices and the constant factor $\frac{1}{c d}$ and by introducing $\tilde{\alpha}=$ $\alpha / d, \tilde{\beta}=\beta / c, \tilde{P}\left(u_{1}, u_{2}\right)=u_{1} u_{2}$. This yields the equation

$$
\left(1+\tilde{\alpha} \tilde{P}^{k}\right) u_{1} \frac{\partial f}{\partial u_{1}}-\left(1+\tilde{\beta} \tilde{P}^{k}\right) u_{2} \frac{\partial f}{\partial u_{2}}+\left(\gamma\left(1+\tilde{\alpha} \tilde{P}^{k}\right)-\mu\left(1+\tilde{\beta} \tilde{P}^{k}\right)\right) f=h,
$$

with $\gamma \in \mathcal{M}=\left\{0, \frac{1}{c}, \ldots, 1-\frac{1}{c}\right\} \subset\left[0,1\left[, \mu \in \mathcal{N}=\left\{0, \frac{1}{d}, \ldots, 1-\frac{1}{d}\right\} \subset\right.\right.$ $\left[0,1\left[\right.\right.$, where now $h=h\left(u_{1}, u_{2}\right)$ is $\tilde{P}$ - $k$-summable.

Now we use Proposition 7.22 to establish the $\tilde{P}$ - $k$-summability of a formal solution $f$ of $(8.7)$ provided $\tilde{\alpha} \neq \tilde{\beta}$.

Consider any direction $\theta$ for which $h$ is $\tilde{P}$ - $k$-summable and which is not congruent to $-\frac{1}{k} \arg (\tilde{\alpha}-\tilde{\beta}) \bmod \frac{\pi}{k} \mathbb{Z}$. Then there is a function $H(\boldsymbol{u}, \tau)$ holomorphic for $\boldsymbol{u} \in D(\mathbf{0} ; \rho)$ and $\tau \in D(0, \delta)$ or $\tau \in V(\theta-\delta, \theta+\delta ; \infty)$ with the properties (1), (2), (3) stated in Proposition 7.22. In particular, there are positive constants $C, A$ such that

$$
|H(\boldsymbol{u}, \tau)| \leq C \exp \left(A|\tau|^{k}\right) \quad \text { if }|\boldsymbol{u}|<\rho, \tau \in(D(0, \delta) \cup V(\theta-\delta, \theta+\delta ; \infty)),
$$

for every $\tau$, we have $J_{\boldsymbol{u}}(H(., \tau)) \in \mathbb{C}\left\{u_{1}\right\}+\mathbb{C}\left\{u_{2}\right\}$, the Laplace transforms

$$
\mathcal{L}(H)(\boldsymbol{u}, t)=k t^{-k} \int_{\arg \tau=\tilde{\theta}} e^{-\tau^{k} / t^{k}} H(\boldsymbol{u}, \tau) \tau^{k-1} d \tau
$$

converge for $\tilde{\theta}$ near $\theta$ and we have $h(\boldsymbol{u})$ as $\tilde{P}$ - $k$-Gevrey asymptotic expansion of $\mathcal{L}(H)(\boldsymbol{u}, \tilde{P}(\boldsymbol{u}))$ in some $\tilde{P}$-sector of angular opening larger that $\frac{2 \pi}{k}$ bisected by $\arg (\tilde{P}(\boldsymbol{u}))=\theta$.

Suppose for a moment that there is a function $F=F(\boldsymbol{u}, \tau)$ with properties similar to $H$ such that $\mathcal{L}(F)(\boldsymbol{u}, \tilde{P}(\boldsymbol{u}))$ is a solution of (8.7) having $f(\boldsymbol{u})$ as its $\tilde{P}(\boldsymbol{u})$-asymptotic expansion. Using classical properties of the Laplace transform we obtain that $u_{r} \frac{\partial f}{\partial u_{r}}(\boldsymbol{u}, \tilde{P}(\boldsymbol{u}))=\mathcal{L}\left(u_{r} \frac{\partial F}{\partial u_{r}}+\right.$ 
$\left.\tau \frac{\partial F}{\partial \tau}\right)(\boldsymbol{u}, \tilde{P}(\boldsymbol{u})), r=1,2$, and $t^{k} \mathcal{L}(G)(\boldsymbol{u}, t)=\mathcal{L}(k I(G))(\boldsymbol{u}, t)$ for $G$ with properties like $F$, where $I(G)(\boldsymbol{u}, \tau)=\int_{0}^{\tau} \sigma^{k-1} G(\boldsymbol{u}, \sigma) d \sigma$. Inserting into (8.9) this yields that $F$ satisfies the following equation

$$
\begin{aligned}
u_{1} \frac{\partial F}{\partial u_{1}}-u_{2} \frac{\partial F}{\partial u_{2}}+k(\tilde{\alpha}-\tilde{\beta})\left(\tau^{k} F\right. & -k I(F))+k \tilde{\alpha} u_{1} \frac{\partial I(F)}{\partial u_{1}}-k \tilde{\beta} u_{2} \frac{\partial I(F)}{\partial u_{2}} \\
& +(\gamma-\mu) F+k(\gamma \tilde{\alpha}-\mu \tilde{\beta}) I(F)=H
\end{aligned}
$$

Conversely, the existence of a solution $F$ of (8.9) with properties analogous to $(1),(2),(3)$ proves the $\tilde{P}$ - $k$-summability of a formal solution $f$ of (8.7), because (8.7) has a unique formal solution except for its constant term in the special case $\ell=\gamma=\mu=0$. This last assertion follows by consideration of the terms of $f$ of lowest valuation. Details are left to the reader.

In order to solve (8.9), we expand $H$ and $F$ into series with respect to $u_{1}, u_{2}$. We have $H(\boldsymbol{u}, \tau)=\sum_{\ell=0}^{\infty} H_{\ell}(\tau) u_{1}^{\ell}+\sum_{\ell=1}^{\infty} H_{-\ell}(\tau) u_{2}^{\ell}$ and want $F(\boldsymbol{u}, \tau)=\sum_{\ell=0}^{\infty} F_{\ell}(\tau) u_{1}^{\ell}+\sum_{\ell=1}^{\infty} F_{-\ell}(\tau) u_{2}^{\ell}$. Equation (8.9) is equivalent to a sequence of equations for the functions $G_{\ell}=I\left(F_{\ell}\right)$

$$
\begin{aligned}
\left(\ell+\gamma-\mu+k(\tilde{\alpha}-\tilde{\beta}) \tau^{k}\right) G_{\ell}^{\prime}+k \tau^{k-1}(\chi \ell-k(\tilde{\alpha}-\tilde{\beta})+\gamma \tilde{\alpha}-\mu \tilde{\beta}) G_{\ell} & =\tau^{k-1} H_{\ell}, \\
G_{\ell}(0) & =0,
\end{aligned}
$$

for $\ell \in \mathbb{Z}$, where $\chi=\tilde{\alpha}$ if $\ell \geq 0, \chi=\tilde{\beta}$ otherwise.

If $\ell+\gamma-\mu \neq 0$, i.e. $\ell \neq 0$ or $\gamma=\mu$, we can write the solutions of $(8.10)$ using the solutions of the corresponding homogeneous equations

$$
U_{\ell}(\tau)=\left(1+\frac{k(\tilde{\alpha}-\tilde{\beta}) \tau^{k}}{\ell+\gamma-\mu}\right)^{-(\chi \ell-k(\tilde{\alpha}-\tilde{\beta})+\gamma \tilde{\alpha}-\mu \tilde{\beta}) /(k(\tilde{\alpha}-\tilde{\beta}))} .
$$

We obtain

$$
\begin{aligned}
G_{\ell}(\tau) & =U_{\ell}(\tau) \int_{0}^{\tau} \frac{\tau^{k-1} H_{\ell}(s)}{\left(\ell+\gamma-\mu+k(\tilde{\alpha}-\tilde{\beta}) s^{k}\right) U_{\ell}(s)} d s, \\
F_{\ell}(\tau) & =\frac{H_{\ell}(\tau)-k(\chi \ell-k(\tilde{\alpha}-\tilde{\beta})+\gamma \tilde{\alpha}-\mu \tilde{\beta}) G_{\ell}}{\ell+\gamma-\mu+k(\tilde{\alpha}-\tilde{\beta}) \tau^{k}} .
\end{aligned}
$$

By our hypothesis on $\theta$, we can assume that $q=\frac{k(\tilde{\alpha}-\tilde{\beta}) \tau^{k}}{\ell+\gamma-\mu}$ is in the sector $|\arg q|<\pi-\delta / 2$ and therefore $\frac{1}{q} \log (1+q)$ is bounded by some constant. This implies that there are constants $K, M$ such that $\left|U_{\ell}(\tau)\right|$ and $1 /\left|U_{\ell}(\tau)\right|$ are bounded by $M e^{K|\tau|^{k}}$ for $\tau \in D(0, \delta) \cup V(\theta-\delta / 2, \theta+$ $\delta / 2 ; \infty)$ and all $\ell \in \mathbb{Z}, \gamma \in \mathcal{M}, \mu \in \mathcal{N}, \ell \neq 0, \gamma \neq \mu$. By (8.8), we have $\left|H_{\ell}(\tau)\right| \leq C \delta^{-|\ell|} e^{A|\tau|^{k}}$ for all $\ell, \gamma, \mu$, and all $\tau$. The above formula for $F_{\ell}$ implies that there is a constant $D$ such that $\left|F_{\ell}(\tau)\right| \leq$ $D \delta^{-|\ell|} e^{(A+2 K+1)|\tau|^{k}}$ for all $\ell, \gamma, \mu, \tau$ in the case $\ell \neq 0, \gamma \neq \mu$. 
Observe that the formula for $F_{\ell}$ remains valid in the exceptional case $\ell=0, \gamma=\mu$, if we replace $U_{0}$ by $\tilde{U}_{0}(\tau)=\tau^{k-\gamma}$. It is straightforward to prove that $\left|F_{0}(\tau)\right| \leq D e^{(A+2 K+1)|\tau|^{k}}$ for a certain $D$ in the finitely many cases $\gamma \in \mathcal{M}, \mu \in \mathcal{N}, \gamma=\mu$.

This implies that

$$
|F(\boldsymbol{u}, \tau)| \leq \frac{4 D}{2-\delta} e^{(A+2 K+1)|\tau|^{k}}
$$

for $\boldsymbol{u} \in D(\mathbf{0} ; \delta / 2), \tau \in D(0, \delta) \cup V(\theta-\delta / 2, \theta+\delta / 2 ; \infty)$. Thus the $\tilde{P}(\boldsymbol{u})$ $k$-summability of the formal solution $f$ of (8.7) is proved.

Going back to the formal solution of equation (8.3) in the case of the monomial $P=x_{1}^{c} x_{2}^{d}$, we obtain its $P$ - $k$-summability provided $\alpha / d \neq \beta / c$. In the terms of the theorem, this condition is equivalent to conditions (2) and (3) and the theorem is proved for monomials $P$.

In a second step, we consider (8.3) with a homogeneous polynomial $P$ that is not a monomial, i.e. the case $a=b$. We can assume that $a=b=$ 1. We apply Theorem 7.24 to the formal solution $f$ of (8.3) that exists according to the assumption of the theorem. Let $\mathcal{M}$ denote the set of zeroes of $P$ in $\mathbb{P}_{\mathbb{C}}^{1}$. If we show that $f \circ b_{\xi}$ is $P \circ b_{\xi}-k$-summable for $\xi \in \mathcal{M}$ then the statement follows in the present case.

It is sufficient to consider the case where $\xi \in \mathbb{C}$, the case $\xi=\infty$ is reduced to the case $\xi=0$ by exchanging $v_{1}$ and $v_{2}, \alpha$ and $\beta, a$ and $b$ respectively.

Now we consider $\tilde{f}=f \circ b_{\xi}$ and the analogously constructed $\tilde{h}, \tilde{P}$, $\tilde{A}, \tilde{B}$. We calculate

$$
\begin{aligned}
\frac{\partial f}{\partial x_{2}}\left(v_{2},\left(\xi+v_{1}\right) v_{2}\right) & =\frac{1}{v_{2}} \frac{\partial \tilde{f}}{\partial v_{1}}\left(v_{1}, v_{2}\right), \\
\frac{\partial f}{\partial x_{1}}\left(v_{2},\left(\xi+v_{1}\right) v_{2}\right) & =\left(\frac{\partial \tilde{f}}{\partial v_{2}}-\frac{\xi+v_{1}}{v_{2}} \frac{\partial \tilde{f}}{\partial v_{1}}\right)\left(v_{1}, v_{2}\right)
\end{aligned}
$$

and obtain analogous formulas for $\tilde{P}$. This leads to the following partial differential equation for $\tilde{f}$.

$$
\begin{aligned}
\left(\left(\xi+v_{1}\right)\right. & \left.\frac{\partial \tilde{P}}{\partial v_{1}}+\alpha \tilde{P}^{k+1}+\tilde{P} \tilde{A}\right) v_{2} \frac{\partial \tilde{f}}{\partial v_{2}} \\
& -\left(v_{2} \frac{\partial \tilde{P}}{\partial v_{2}}+(\alpha+\beta) \tilde{P}^{k+1}+\tilde{P}(\tilde{A}+\tilde{B})\right)\left(\xi+v_{1}\right) \frac{\partial \tilde{f}}{\partial v_{1}}=\tilde{h}
\end{aligned}
$$

where now the $v_{2}$-valuation of $\tilde{A}$ and $\tilde{B}$ is larger than $k g, g=w(P)$, and $\tilde{P}\left(v_{1}, v_{2}\right)=P\left(v_{2},\left(\xi+v_{1}\right) v_{2}\right)=v_{2}^{g} Q\left(v_{1}\right)$ with some polynomial $Q$ vanishing at the origin. 
In order to reduce $\tilde{P}$ to a monomial, we consider a holomorphic function $\phi$ such that $Q\left(\phi\left(v_{1}\right)\right)=v_{1}^{c}$, where $c$ is the multiplicity of $v_{1}=0$ as a zero of $Q$. Then replacing $v_{1}$ by $\phi\left(v_{1}\right)$, multiplying by $v_{1} \phi^{\prime}\left(v_{1}\right)$, and dividing by $\xi+\phi\left(v_{1}\right)$, we obtain a new equation for $\bar{f}\left(v_{1}, v_{2}\right)=\tilde{f}\left(\phi\left(v_{1}\right), v_{2}\right)$ with $\bar{P}=v_{1}^{c} v_{2}^{d}$.

$$
\begin{aligned}
&\left(v_{1} \frac{\partial \bar{P}}{\partial v_{1}}+\frac{v_{1} \phi^{\prime}\left(v_{1}\right)}{\xi+\phi\left(v_{1}\right)}\left(\alpha \bar{P}^{k+1}+\bar{P} \bar{A}\right)\right) v_{2} \frac{\partial \bar{f}}{\partial v_{2}} \\
&-\left(v_{2} \frac{\partial \bar{P}}{\partial v_{2}}+(\alpha+\beta) \bar{P}^{k+1}+\bar{P}(\bar{A}+\bar{B})\right) v_{1} \frac{\partial \bar{f}}{\partial v_{1}}=\bar{h}
\end{aligned}
$$

where $\bar{h}\left(v_{1}, v_{2}\right)=\frac{v_{1} \phi^{\prime}\left(v_{1}\right)}{\xi+\phi\left(v_{1}\right)} \tilde{h}\left(\phi\left(v_{1}\right), v_{2}\right)$ and $\bar{A}, \bar{B}$ are certain convergent series containing only terms of $v_{2}$-valuation larger than $k d$. Choosing the quasi-homogeneous valuation appropriately, more precisely $a=1$ and $b$ sufficiently large, we arrive at an equation of the form (8.3) with the monomial $P=x_{1}^{c} x_{2}^{g}$. In the case $\xi \neq 0$, the factors $\alpha, \beta$ replaced by $\alpha+\beta, 0$, and $f \circ b_{\xi}$ is $P \circ b_{\xi}-k$-summable if $\alpha+\beta \neq 0$; this is satisfied by condition (1). In the case $\xi=0$, these factors have to be replaced by $(\alpha+\beta, \alpha)$. As $c=\mu_{0}>0$ and $d=g$ here, we obtain that $f \circ b_{0}$ is $P \circ b_{0}-k$-summable if $(\alpha+\beta) / g \neq \alpha / \mu_{0}$. This condition is equivalent to (2). In the case $\xi=\infty$ the reduction to the case $\xi=0$, that is exchange $x_{1}$ and $x_{2}$ etc., proves that $f \circ b_{\infty}$ is $P \circ b_{\infty}-k$-summable if $(\alpha+\beta) / g \neq \beta / \mu_{\infty}$. This is condition (3). Thus the theorem is proved for homogeneous non-monomial $P$.

In a third and last step, we reduce a quasi-homogeneous non-monomial case to a homogeneous case by the ramifications $x_{1}=v_{1}^{a}, x_{2}=v_{2}^{b}$. It suffices to use Proposition 7.26 twice. The ramifications preserve the form (8.3) of the equation, only $\alpha, \beta$ are replaced by $b \alpha, a \beta$, it is homogeneous for $w$ satisfying $w\left(v_{1}\right)=w\left(v_{2}\right)=1$ and $\mu_{0}, \mu_{\infty}$ are replaced by $b \mu_{0}, a \mu_{\infty}$, but $g$ remains unchanged. This yields the conditions of the theorem for $P$ - $k$-summability in the present case.

\section{References}

[AO] R. C. Ackerberg and R. E. O'Malley, JR., Boundary layer problems exhibiting resonance, Studies in Appl. Math. 49(3) (1970), 277-295. DOI: 10.1002/sapm1970493277.

[AHV] J. M. Aroca, H. Hironaka, and J. L. Vicente, "The Theory of the Maximal Contact", Memorias de Matemática del Instituto "Jorge Juan" 29, Instituto "Jorge Juan" de Matemáticas, Consejo Superior de Investigaciones Científicas, Madrid, 1975.

[Bal] W. BALSER, "From Divergent Power Series to Analytic Functions", Theory and application of multisummable power series, Lecture Notes in Mathematics 1582, Springer-Verlag, Berlin, 1994. DOI : 10.1007/BFb0073564. 
[BM] E. Bierstone and P. D. Milman, Semianalytic and subanalytic sets, Inst. Hautes Études Sci. Publ. Math. 67 (1988), 5-42.

[Can] M. Canalis-Durand, Solutions Gevrey d'équations différentielles singulièrement perturbées, Mémoire d'habilitation à diriger des recherches, Univ. AixMarseille III (1999).

[CMS] M. Canalis-Durand, J. Mozo-Fernández, and R. Schäfke, Monomial summability and doubly singular differential equations, J. Differential Equations 233(2) (2007), 485-511. DOI : 10.1016/j.jde.2006.11.005.

[CS] M. Canalis-Durand and R. Schäfke, Divergence and summability of normal forms of systems of differential equations with nilpotent linear part, Ann. Fac. Sci. Toulouse Math. (6) 13(4) (2004), 493-513.

[Car] S. A. Carrillo Torres, Monomial multisummability through BorelLaplace transforms. Applications to singularly perturbed differential equations and Pfaffian systems, PhD Thesis, University of Valladolid (2016). http://uvadoc.uva.es.

[CM] S. A. Carrillo and J. Mozo-Fernández, Tauberian properties for monomial summability with applications to Pfaffian systems, J. Differential Equations 261(12) (2016), 7237-7255. DOI: 10.1016/j.jde.2016.09.017.

[CE] L. P. COOK AND W. ECKHAus, Resonance in a boundary value problem of singular perturbation type, Studies in Appl. Math. 52(2) (1973), 129-139. DOI : $10.1002 /$ sapm1973522129.

[Gal] A. Galligo, Théorème de division et stabilité en géométrie analytique locale, Ann. Inst. Fourier (Grenoble) 29(2) (1979), 107-184. DOI: 10.5802/aif. 745.

[GS] R. GÉrard and Y. Sibuya, Étude de certains systèmes de Pfaff avec singularités, in: "Équations différentielles et systèmes de Pfaff dans le champ complexe" (Sem., Inst. Rech. Math. Avancée, Strasbourg, 1975), Lecture Notes in Math. 712, Springer, Berlin, 1979, pp. 131-288. DOI : 10.1007/BFb0062810.

[Lak] W. D. LAKIN, Boundary value problems with a turning point, Studies in Appl. Math. 51(3) (1972), 261-275. DOI : 10.1002/sapm1972513261.

[Li] P. LI, Examples of monomial summability for the formal solutions of certain PDE, Manuscript, Strasbourg (2008).

[Lor] F. LORAY, Réduction formelle des singularités cuspidales de champs de vecteurs analytiques, J. Differential Equations 158(1) (1999), 152-173. DOI : 10.1016/S0022-0396 (99)80021-7.

[Maj] H. MAJIma, "Asymptotic Analysis for Integrable Connections with Irregular Singular Points", Lecture Notes in Mathematics 1075, Springer-Verlag, Berlin, 1984. DOI : 10.1007/BFb0071550.

[MR] J. MARTinet AND J.-P. RAmis, Classification analytique des équations différentielles non linéaires résonnantes du premier ordre, Ann. Sci. École Norm. Sup. (4) 16(4) (1983), 571-621 (1984). DOI : 10.24033/asens.1462.

[Olv] F. W. J. Olver, "Asymptotics and Special Functions", Computer Science and Applied Mathematics, Academic Press [A subsidiary of Harcourt Brace Jovanovich, Publishers], New York-London, 1974.

[Ram1] J.-P. Ramis, Dévissage Gevrey, in: "Journées Singulières de Dijon" (Univ. Dijon, Dijon, 1978), Astérisque 59-60, Soc. Math. France, Paris, 1978, pp. 4, 173-204. 
[Ram2] J.-P. RAmis, Les séries $k$-sommables et leurs applications, in: "Complex Analysis, Microlocal Calculus and Relativistic Quantum Theory" (Proc. Internat. Colloq., Centre Phys., Les Houches, 1979), Lecture Notes in Phys. 126, Springer, Berlin-New York, 1980, pp. 178-199. DOI : 10.1007/3540-09996-4.

[RSW] J.-P. Rolin, P. Speissegger, And A. J. Wilkie, Quasianalytic DenjoyCarleman classes and o-minimality, J. Amer. Math. Soc. 16(4) (2003), 751-777. DOI : $10.1090 / \mathrm{S} 0894-0347-03-00427-2$.

[Sib1] Y. SibuYA, "Linear Differential Equations in the Complex Domain: Problems of Analytic Continuation", Translated from the Japanese by the author, Translations of Mathematical Monographs 82, American Mathematical Society, Providence, RI, 1990.

[Sib2] Y. SibuYA, Gevrey property of formal solutions in a parameter, in: "Asymptotic and Computational Analysis" (Winnipeg, MB, 1989), Lecture Notes in Pure and Appl. Math. 124, Dekker, New York, 1990, pp. 393-401.

[Ste] J. Stevens, "Deformations of Singularities", Lecture Notes in Mathematics 1811, Springer-Verlag, Berlin, 2003. DOI : 10.1007/b10723.

[Sto] L. Stolovitch, Classification analytique de champs de vecteurs 1-résonnants de $\left(\mathbf{C}^{n}, 0\right)$, Asymptotic Anal. 12(2) (1996), 91-143. DOI: 10.3233/ASY1996-12202.

[Was1] W. Wasow, "Asymptotic Expansions for Ordinary Differential Equations", Pure and Applied Mathematics XIV, Interscience Publishers John Wiley \& Sons, Inc., New York-London-Sydney, 1965.

[Was2] W. WAsow, Topics in the theory of linear ordinary differential equations having singularities with respect to a parameter, Preprint, Université Louis Pasteur, Strasbourg (1978).

Jorge Mozo Fernández

Departamento de Álgebra, Análisis Matemático, Geometría y Topología, Facultad de Ciencias, Campus Miguel Delibes, Universidad de Valladolid, Paseo de Belén, 7, 47011 Valladolid, Spain

E-mail address: jorge.mozo@uva.es

Reinhard Schäfke

Institut de Recherche Mathématique Avancée, U.F.R. de Mathématiques et Informatique, Université de Strasbourg et C.N.R.S., 7, rue René Descartes, 67084 Strasbourg cedex, France

E-mail address: schaefke@unistra.fr

Primera versió rebuda el 2 de febrer de 2017, darrera versió rebuda el 27 de novembre de 2017. 